UNIVERSIDADE DE BRASILIA - UNB

INSTITUTO DE GEOCIÊNCIAS - IG

\title{
DETERMINAÇÃO DE PARÂMETROS DE FONTE DE EVENTOS LOCAIS E REGIONAIS POR INVERSÃO DE FORMAS DE ONDAS
}

\author{
Dissertação de Mestrado $\mathrm{n}^{\circ} 72$ \\ Juraci Mário de Carvalho
}

Orientador: Prof. Dr. Lucas Vieira Barros 


\title{
DETERMINAÇÃO DE PARÂMETROS DE FONTE DE EVENTOS LOCAIS E REGIONAIS POR INVERSÃO DE FORMAS DE ONDAS
}

\author{
Juraci Mário de Carvalho \\ Dissertação de mestrado $\mathrm{N}^{\circ} 72$
}

Orientado: Prof. Dr. Lucas Vieira Barros

\author{
Banca Examinadora: \\ Prof. Dr. Lucas Vieira Barros (UnB) \\ Prof. Dr. Marcelo Peres Rocha (UnB) \\ Prof. Dr. Marcelo Sousa de Assumpção (USP) \\ Programa de Pós-graduação em Geociências Aplicadas \\ Área de concentração Geofísica Aplicada
}

Brasília-DF, Fevereiro de 2015 


\section{REFERÊNCIA}

Carvalho, J. M. 2015.

DETERMINAÇÃO DE PARÂMETROS DE FONTE DE EVENTOS LOCAIS E REGIONAIS POR INVERSÃO DE FORMAS DE ONDAS

Universidade de Brasília, Instituto de Geociências, Dissertação de Mestrado.

Palavras-chave: Parâmetros de fonte, inversão de formas de ondas, mecanismo focal, momento tensor, esforços. 
"When Richards agreed to work as my coauthor, I thought that I could enter the mainstream of seismology at last. Though I could not complete anything alone and always needed someone's help, I made many cordial friends instead".

Keiiti Aki 


\section{AGRADECIMENTOS}

Agradeço à minha mulher e aos meus filhos Rodrigo Fontenele Carvalho, Victor Fontenele Carvalho e Bruno Fontenele Carvalho, pelo apoio e pela compreensão do tempo de convívio muitas vezes sacrificado para realização deste trabalho. Agradeço especialmente a minha esposa Jackeline Portela Fontenele Carvalho pelo apoio, carinho, compreensão e incentivo.

Ao Prof. Dr. Lucas Barros, meu orientador e amigo, pela confiança. Agradeço por ter acreditado no meu potencial e por ter me acompanhado durante toda essa caminhada.

Ao Prof. Dr. Jiří Zahradník por sua valiosa ajuda e paciência durante o processo de desenvolvimento dessa dissertação. Um amigo que ganhei durante essa jornada.

Ao Prof. Dr. Marcelo Assumpção e Hans Agurto Detzel pelo suporte, informações e scripts de mapas.

Ao Prof. Dr. Jose E. P. Soares pela disponibilização dos dados das estações sismográficas RETs adquiridos no âmbito do projeto: Estudos sobre $O$ Lineamento Transbrasiliano (Rede de Estudos Tectônicos-Petrobras).

Agradeço aos meus colegas de trabalho, Darlan Portela Fontenele, Vinicius Martins, Daniel Caixeta, Diogo Farrapo e outros que me ajudaram no decorrer do mestrado com dados, instrumentação, scripts de mapas, correção de textos e apoio pessoal.

Agradeço aos meus professores do Instituto de Geociências da UnB com quem muito aprendi durante essa jornada.

Agradeço ao Instituto de Geociências da UnB, SIS-UnB e IAG-USP pela disponibilização de recursos, dados da rede sismográfica brasileira e de outras estações sismográficas. 


\section{SUMÁRIO}

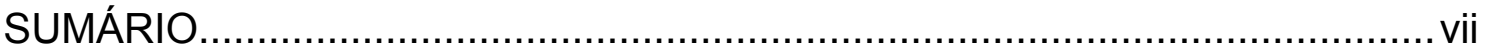

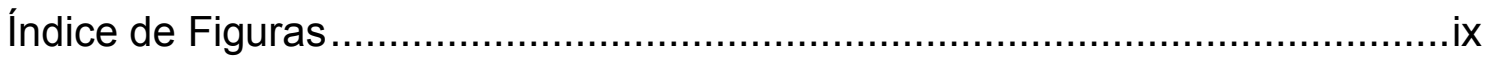

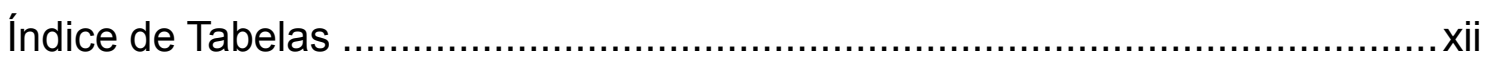

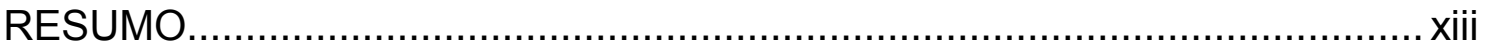

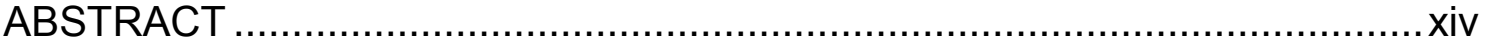

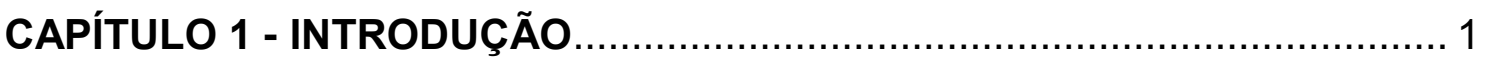

1.1. A importância do tema ............................................................. 1

1.2. Objetivos da dissertação ......................................................... 3

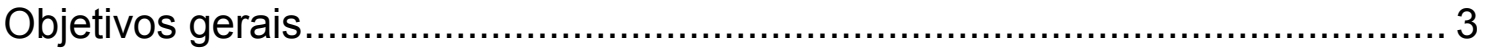

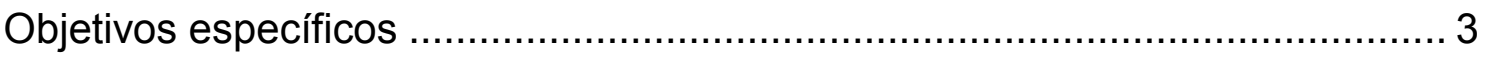

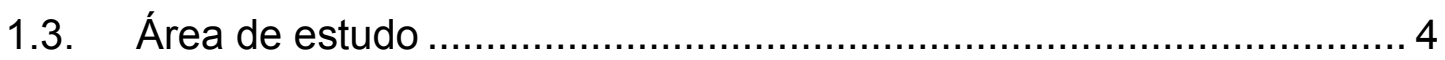

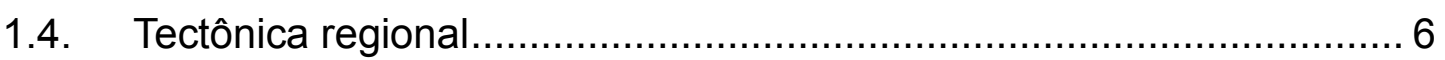

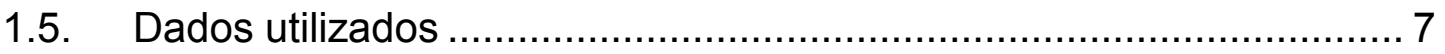

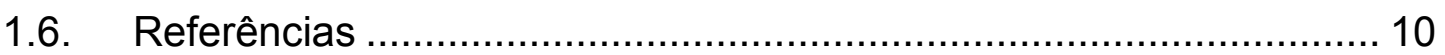

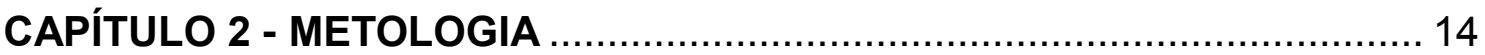

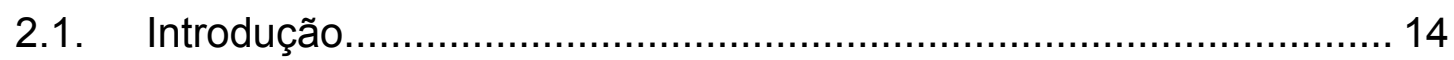

2.2. Parâmetros de fonte .......................................................... 15

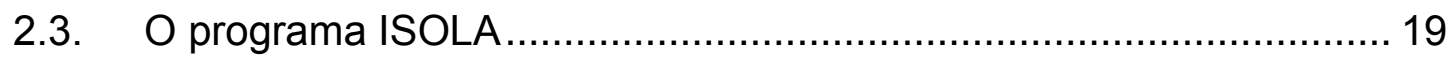

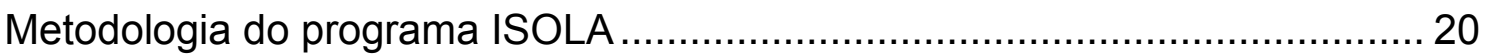

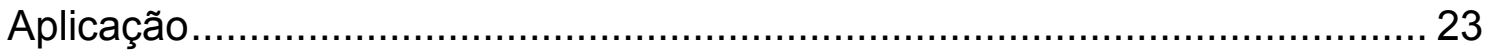

2.4. Inversão de dados de mecanismos focais ................................... 24

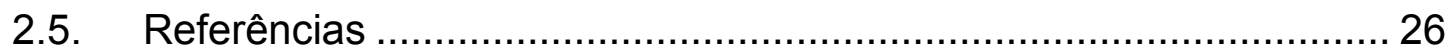

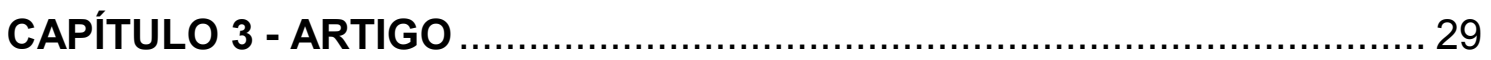

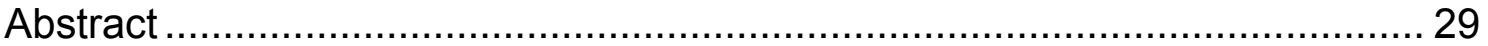




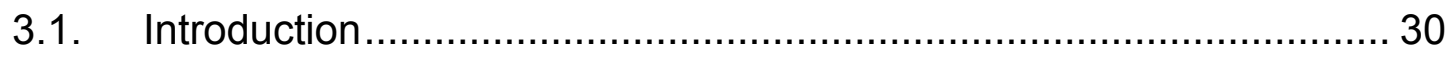

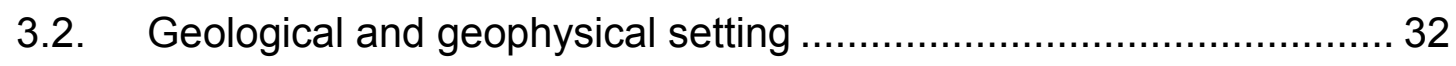

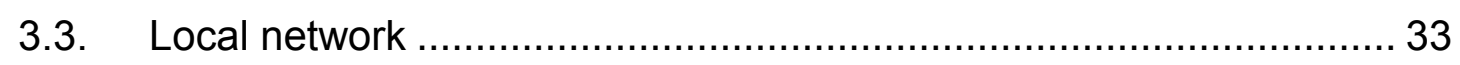

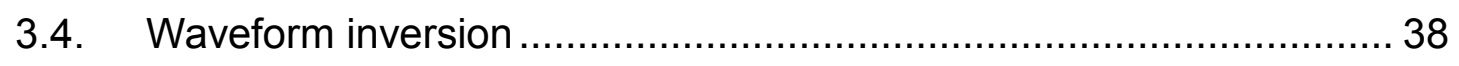

3.5. Discussion and conclusions........................................................ 49

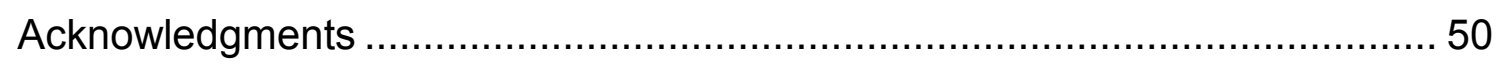

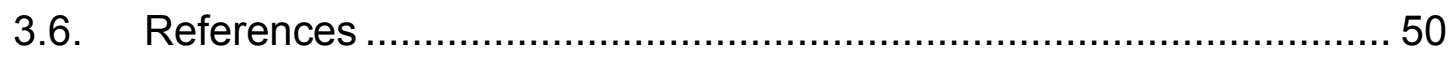

CAPITULO 4 - INVERSAO DO EVENTO PRINCIPAL ................................. 55

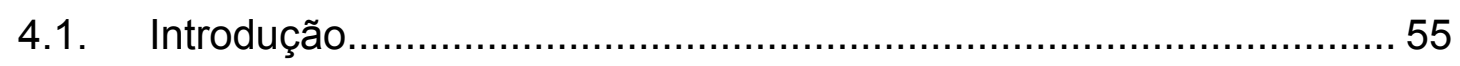

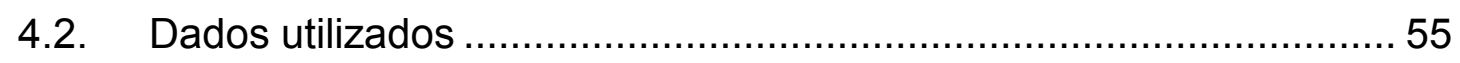

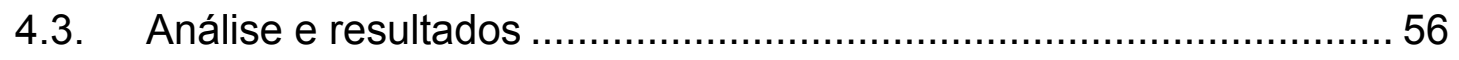

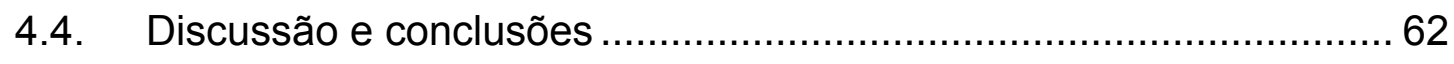

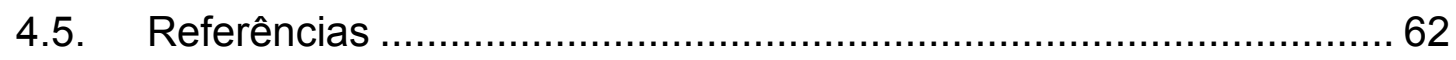

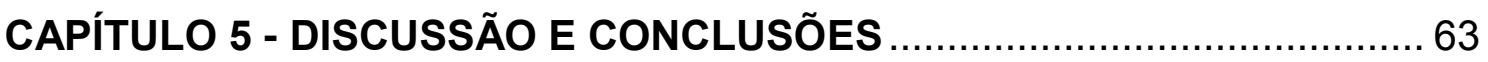

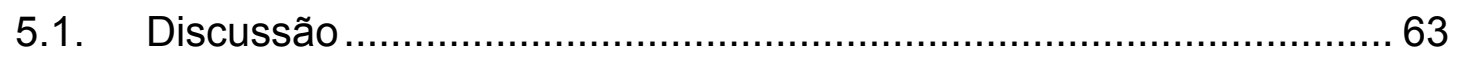

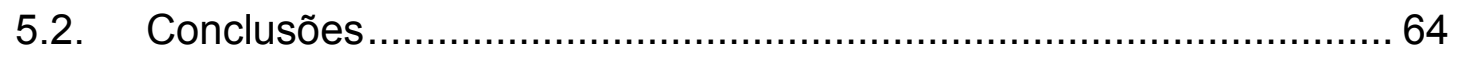

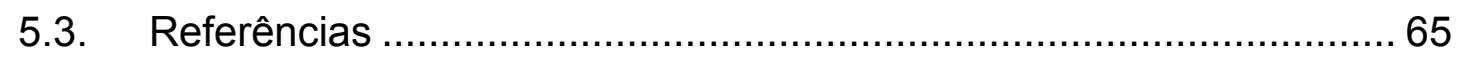

Trabalhos produzidos no âmbito desta dissertação ……………………….... 66

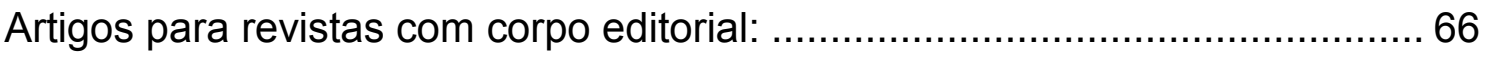

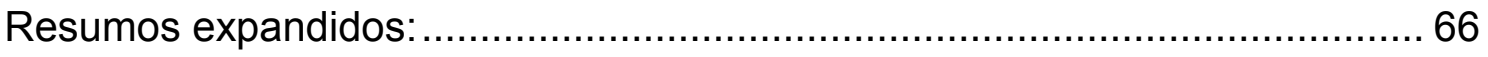

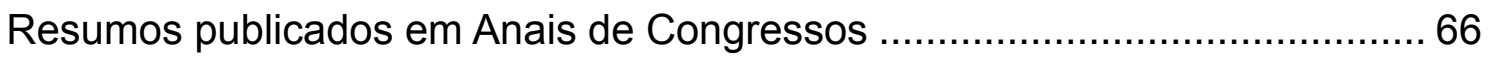

Formas de ondas (sintéticas e observadas) obtidas na inversão das onze

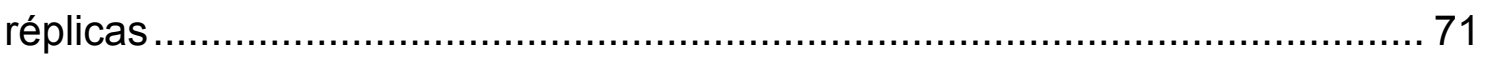




\section{Índice de Figuras}

Figura 1.1 - Faixa sísmica Goiás-Tocantins (retângulo amarelo) e estruturas do Lineamento Transbrazilianos (LTB). O quadrado branco mostra a área de estudo e a estrela indica o evento principal de Mara Rosa. As províncias geológicas são: PTO (Província Tocantins); BPR (Bacia do Paraná); CAM (Craton Amazônoniano); BPB (Bacia do Parnaíba) e CSF (Craton São Francisco).

Figura 1.2 - Rede sismográfica de Mara Rosa (triângulos) e epicentros dos eventos melhores registrados da atividade de réplicas (53, círculos vermelhos e azuis). Os 11 círculos azuis representam os eventos para os quais se realizou inversões. A estrela amarela denota o epicentro do evento principal, $5.0 \mathrm{~m}_{b}$ de 08/10/2010.

Figura 1.3 - Formas de ondas, nas componentes verticais, do evento 10 da Tabela 1.3 registrados pelas estações MR03, MR04, MR07, MR08, MR09, MR10 e MR11. Os dados são em velocidade (contagens digitais) e filtro 1.5 a $2.0 \mathrm{~Hz}$. O Traço vertical marca o tempo de origem (TO). 10

Figura 1.4 - Estimativa da direção dos esforços na placa Sul Americana a partir de dados de Mecanismos Focais, breakouts e medidas in-situ. As cores das barras representam: amarelo - tração e azul - compressão. Quadrado branco mostra a área de estudo. Figura de Assumpção et al. (2008).Erro! Indicador não definido.

Figura 2.1 - Diagrama em blocos do sistema representando a fonte, $x(t)$, o meio, $g(t)$ e o instrumento, $i(t)$, no domínio do tempo (a) e no domínio da frequência (b). 14

Figura 2.2 - Tipos de forças que podem gerar deslocamento observados a longas distâncias (far field), provenientes da ruptura de uma falha (Havskov \& Ottemöller, 2010).

Figura 2.3 - Nove pares de forças que representam o momento tensor. E.g., Mxy é uma força dupla no plano xy agindo na direção do eixo $\boldsymbol{x}$ (Stein \& Wysession 2003).

Figura 2.4 - Parâmetros de uma falha. A orientação do plano da falha é definida pelos ângulos dip e strike. $\boldsymbol{O}$ movimento relativo dos blocos é definido pelo ângulo do vetor slip (rake) (Stein \& Wysession, 2003).

Figura 2.5 - O diagrama à esquerda mostra a esfera focal e a projeção dos raios sísmicos em direção às estações. O diagrama da direita mostra os beachballs das falhas do tipo strike slip e normal. Os quadrantes hachurados, em preto e branco, correspondem às áreas de compressão e tensão, respectivamente (Havskov \& Ottemöller, 2010).

Figura 2.6 - Tipos de falhas e respectivos esforços (P\&T) necessários para sua ruptura (http://earthquake.usgs.gov/learn/topics/beachball.php). 18

Figura 2.7 - Padrão de irradiação (esquerdo amplitudes e direito direção) das ondas $P$ (a) e $S(b)$, considerando uma fonte pontual do tipo Double Couple (Stein \& Wysession, 2003). 
Figura 2.8 - Momentos tensores elementares usados na inversão do momento tensor completo (Kikuchi \& Kanamori, 1991).

Figure 3.1 - Seismicity (red circles) of Goiás-Tocantins Seismic Zone up to 2014 (Brazilian Seismic Bulletin - SISBRA), geological map with structures of the TransBrasiliano Lineament (CPRM, 2004) and regional seismic stations (blue triangles).The focal mechanisms of the 2000 and 2010 events are taken from Assumpção et al. (2014) and from Barros et al. (2014), respectively...33

Figure 3.2 - Mara Rosa local seismographic network (triangles) and local seismicity from Oct/2010 to Jun/2011 (magnitudes <2.0). Red circles are the 53 best located events. The 11 events shown in blue are inverted for their focal mechanism in this paper (Two events overlapped to the same location). The star denotes the mainshock $5.0 m_{b}$ of October 8, 2010.

Figure 3.3 - Velocity model adopted in this paper for waveform inversion. (Derived from Soares et al., 2006).

Figure 3.4 - Waveforms quality check showing data problems, including: a) excessive cultural noise present at all components (Ev10, MR09, filtered 1.5-2.0 Hz); b) monochromatic noise present at all components (EV6, MR3, filtered 1.5$2.0 \mathrm{~Hz}) ; \mathrm{c})$ instrumental disturbance present at all components (Ev6, MR08, raw data); and d) instrumental disturbance present at the horizontal components (Ev6, MR11, raw data).

Figure 3.5 - Comparison between the observed (black) and synthetic (red) waveforms. The example refers to Event 9 of Tables 3.2 and 3.3. The waveform match is quantified by the variance reduction values per station components (blue numbers). The horizontal time axis is in seconds, $t=50 \mathrm{sec}$ corresponds to 10 seconds after the origin time. The vertical axis indicates the displacement amplitude in meters. 39

Figure 3.6 - The uncertainty of the focal mechanisms calculated in the present paper, characterized by the scatter plots of nodal lines and the corresponding confidence intervals.

Figure 3.7 - Comparison of the focal mechanism solutions: (a) the composite first-motion polarity solution; small symbols are clear polarity data from aftershocks recorded by the local network; large symbols are clear polarity data of the mainshock at regional stations. (b) the waveform inversion of the present paper (Events 1 to 10 of Table 3.3). The $P \&$ T axes, the red line and shading in panel $(b)$ is the same solution as in panel $(a)$. 44

Figure 3.8 - Focal mechanisms derived in this paper and the corresponding horizontal projections of their $P$ axes (red bars) for the investigated events of Table 3. Blue bar in the inset indicates the NW-SE regional compression according to Caproni \& Armelin (1990).

Figure 3.9 - The HypoDD relocated aftershocks in map view (top) and in a vertical crosssection (bottom), compared to a plane best fitting their distribution (strike $244^{\circ}$, dip $\left.26^{\circ}\right)$ after Barros et al. (2014). The cross-section is plotted (NNWSSE) perpendicularly to the $244^{\circ}$ strike. The red squares and pink circles indicate the shallower and deeper events, respectively, choosing the limit between them tentatively at a depth of $1.3 \mathrm{~km}$. One station (triangle) is shown as a reference. 46 
Figure 3.10 - Stress inversion based on focal mechanisms of Events 1 to 10. (a) P (red) \& $T$ (blue) axes and the stress directions $\sigma 1, \sigma 2$ and $\sigma 3$ (green). (b) Mohr's circle with the events shown by blue crosses. (c) Relative uncertainty of the stress axes resulting from random perturbation of the input data

Figura 4.1 -Estações com formas de ondas utilizadas na inversão CAN3, SSV2 e BDFB (triângulos vermelhos) e estações usadas apesas as polaridades da fase (P) SFA1, JAN7, MANle RET2, 3, 4, 8 e 9 (triângulos azuis). A estrela denota o epicentro do evento principal de MR.

Figura 4.2 - Sinais no dominio do tempo das estações BDFB, CAN3 e SSV2 filtrados na banda de $0.05 \mathrm{~Hz}-0.13 \mathrm{~Hz}$. Observa-se claramente a existência de energia nos dados filtrados nas três estações. O instrumental da estação BDFB é de banda larga $(120 \mathrm{sec}-50 \mathrm{~Hz})$.

Figura 4.3 - correlação entre a posição da fonte (em profundidade) e o tempo de origem do centroide momento tensor. A origem do evento no ISOLA A maior correlação (beachball vermelho) foi obtida para a fonte número 4 (beachball vermelho). e a fonte adotada foi a de número 6 (círculo amarelo) com maior porcentagem de double-couple (DC\%). 58

Figura 4.4 - Correlação dos mecanismos focais pela profundidade. A fonte 4 (seta azul) apresenta uma correlaçao ligeiramente maior que a fonte 6 (seta vermelha). Entretanto, a fonte 6 tem maior porcentagem de double-couple $(D C \%=66)$ e maior profundiade $(6 \times 0.2 \mathrm{~km}=1.2 \mathrm{~km})$, mais próxima da profundidade do evento GT5 $(h=1.3 \mathrm{~km})$. 58

Figura 4.5 - Correlaçao entre sismogramas sintéticos (traços vermelhos) e observados (traços pretos) registrado em tres estações regionais (CAN3, SSV2 e BDFB). A correlação das estações CAN3 (NS e EW) e BDFB (NS) são baixas nessas componentes devido principalmente às longas distâncias entre fonte $e$ estações e baixa resolução do modelo crustal adotado.

Figura 4.6 - resultados da inversão de formas de ondas do sismo principal de Mara Rosa, 8 Outubro 2010, magnitude $5.0 \mathrm{mb}$ e intensidade VI (MM). As estações utilizadas na inversão foram CAN3 (CAN), SSV2 (SSV) e BDFB (BDFB). .. 60

Figura 4.7 - mecanismo focal do evento principal de Mara Rosa obtido por inversão de formas de ondas, utilizando três estações regionais (CAN3, SSV2 e BDFB) e polaridades das estações SSV2, CAN3, MAN1, IGA2, JAN7, SFA1, RET2, RET3, RET4, RET8, RET9 e BDFB. U (up) e D (down) são polaridades da primeira chegada da fase (P). A maioria das polaridades ou está em concordância ou próximas dos planos nodais do mecanismo focal resultante. 


\section{Índice de Tabelas}

Tabela 1.1 - Características dos instrumentos sismográficos utilizados na rede local de Mara Rosa. A estação MR5 foi remandejada para MR10. As estações funcionaram entre outubro de 2010 a maio de 2011.

Tabela 1.2 - Características dos instrumentos sismográficos utilizados na rede regional de Mara Rosa. As estações funcionaram durante todo o período de monitoramento e continuam em operação.

Tabela 1.3 - Lista de eventos selecionados para a inversão. 10

Table 3.2 - Velocity model $(V p / V s=1.70)$ 35

Table 3.3 - Events selected for the waveform inversion.

Table 3.4 - Summary of the results. See also Figure 3.6. All events were inverted in the frequency range 1.5-2.0 $\mathrm{Hz}$. The boxes marked with blue color indicate problematic cases. For each event we calculate the angular deviation with respect to the composite focal mechanism (in terms of the Kagan angle), and present it in the last column as Similarity 43

Table 3.5 - Principal stress axes and the optimally oriented faults derived from Events 1 to 10. 48

Tabela 4.2 - Modelo de velocidades crustais utilizado na inversão. 56

Tabela 4.3 - Resumo dos parâmetros da inversão com o pacote ISOLA. A primeira linha mostra os parâmetros do mecanismo focal composto e a segunda os parâmetros obtidos pela inversão. 60 


\section{RESUMO}

No Brasil, pouco se conhece sobre a distribuição dos esforços intraplaca devido à baixa sismicidade associada à baixa densidade de estações sismográficas. Apenas muito recentemente esse quadro tem mudado devido à implantação da Rede Sismográfica Brasileira (RSBR). Os estudos de mecanismo focal se iniciaram apenas, em 1978, com o trabalho pioneiro de Mendiguren e Richter.

Este trabalho dá uma contribuição a esses estudos pois mostra que é possível obter, de forma confiável, mecanismos focais de microtremores por inversão de formas de ondas. Foram obtidos os parâmetros de fonte do sismo principal de Mara Rosa (5.0 mb, VI MM, em 08/10/2010) e de onze de suas réplicas registradas por uma rede local de oito estações com instrumentos de período curto $(1 \mathrm{~Hz}-100 \mathrm{~Hz}$ ) e período longo (30 s $-100 \mathrm{~Hz}$ ), ambos amostrados a $200 \mathrm{sps}$ com distâncias epicentrais menores que $8 \mathrm{~km}$. Essas distâncias permitiram modelar frequências relativamente altas $(1.5 \mathrm{~Hz}$ a 2.0 $\mathrm{Hz}$ ). A banda de frequência da inversão foi determinada, na parte inferior, pela razão SNR e na parte superior pela frequência de esquina (corner frequency).

A seleção dos dados envolveu uma inspeção rigorosa nas formas de ondas para remover traços com perturbações instrumentais. Na avaliação da confiabilidade dos resultados foram usados vários parâmetros de controle de qualidade, tais como VR (Variance Reduction), CN (Condition Number), DC\% (porcentagem Double Couple), SNR (relação sinal-ruído), concordância de polaridades e computação da incerteza teórica ou dispersão dos resultados.

O melhor ajuste entre sintético e observado foi obtido com uma busca iterativa no espaço e no tempo. A posição do centroide foi inicialmente fixada no epicentro, variando a profundidade de $0.4 \mathrm{~km}$ até $2.0 \mathrm{~km}$, em passos de 0.2 $\mathrm{km}$ e tempo de origem variando em $\pm 1,0$ segundo, em passos de 90 milissegundos, permitindo a otimização do momento tensor.

Foram obtidos bons resultados para todos os eventos estudados, com a maioria dos parâmetros de controle de qualidade dentro dos limites estabelecidos. As inversões das formas de ondas das onze réplicas resultaram em falhas com strike variando de $188^{\circ}$ a $280^{\circ}$, consistentes com estudos anteriores. A inversão dos mecanismos focais das replicas mostrou que 0 evento de Mara Rosa ocorreu em uma falha com direção $264^{\circ}$ e mergulho de $16^{\circ}$. Os esforços principais ( $\sigma 1, \sigma 2$ e $\sigma 3$ ) encontrados foram $155^{\circ}, 63^{\circ}$ e $312^{\circ}$ com $\sigma 1$ e $\sigma 2$ horizontais, com plunge de $15^{\circ}$ e $6^{\circ}$, respectivamente, $\sigma 3$ vertical com inclinação de $74^{\circ}$, compatível com modelo de falha inversa encontrada para o evento de Mara Rosa.

Palavras-chave: Mara Rosa, Parâmetros de fonte, inversão de formas de ondas, mecanismo focal, momento tensor, inversão de mecanismo focal. 


\section{ABSTRACT}

In Brazil, little is known about intraplate tectonic stress distribution due to low seismicity associated with low density of seismographic stations. Only recently, this situation has changed because of the implementation of the Brazilian Seismographic Network (RSBR). Studies of focal mechanism in Brazil began only in 1978 with the pioneering work of Mendiguren and Richter.

This paper gives a contribution to these studies in showing that it is possible to obtain reliably, focal mechanisms of micro-earthquakes by waveforms inversion. We obtained the source parameters of the main earthquake Mara Rosa (5.0 mb MM VI, on 08/10/2010) and eleven of its aftershocks detected by a local network of eight stations (distances up to $8 \mathrm{~km}$ ) equipped with short period and broadband sensors. Thanks to the short epicentral distances, we are able to model the complete wavefield up to the relatively high frequencies $(2.0 \mathrm{~Hz})$. The frequency band of the inversion has been determined, at the lower side by the SNR ratio and at high end by the event corner frequency.

Prior to the inversion, a thorough inspection to the waveforms was done to remove data with instrumental disturbances, electronic noise, excessive cultural noise and/or any kind of equipment malfunction, which could bias the inversion results. To assess the results reliability, we used several qualitycontrol parameters, such as VR (variance reduction), CN (condition number), DC\% (double couple percentage), SNR (signal-to-noise ratio), the first-motion polarity agreement and the focal mechanism uncertainty described by the Kagan-angle dispersion.

We investigate the focal mechanisms using the ISOLA program, based on the least-squares calculation of the moment tensor and grid search of the centroid position and time. The grid-searched centroid positions (trial sources) was situated below the fixed epicenter, ranging the depths from $0.4 \mathrm{~km}$ to 2.0 $\mathrm{km}$; in steps of $0.2 \mathrm{~km}$ and origin time ranging from +1.0 to -1.0 second, in steps of 90 milliseconds allowing the optimization of the deviatoric moment tensor.

Good results were obtained for the eleven studied events, with most quality-control parameters meeting the optimum values established in previous studies. The inversion of the waveform of the eleven aftershocks resulted in faults striking from $188^{\circ}$ to $280^{\circ}$, consistent with previous studies. The stress inversion from focal mechanisms showed that the Mara Rosa main event occurred on a fault striking at $264^{\circ}$ and dip $16^{\circ}$. The direction of the obtained principal stresses $(\sigma 1, \sigma 2$ and $\sigma 3)$ are $155^{\circ}, 312^{\circ}$ and $63^{\circ}$ respectively. The $\sigma 1$ and $\sigma 2$ axes are nearly horizontal $\left(15^{\circ}\right.$ and $\left.6^{\circ}\right)$; the $\sigma 3$ axis is nearly vertical $\left(74^{\circ}\right)$, corresponding to the reverse faulting regime. 
KeyWords: source parameters, focal mechanism, waveform inversion, double-couple percentage, deviatoric moment tensor. 


\section{CAPÍTULO 1 - INTRODUÇÃO}

\subsection{A importância do tema}

O Brasil está localizado no Interior Continental Estável (ICE) da placa Sul Americana e apresenta uma sísmicidade relativamente baixa, inclusive menor do que aquela observada em outras regiões intraplacas similares, como é o caso do leste dos Estados Unidos, da Índia e da Austrália (Barros, et al., 2009). Com apenas duas dezenas de eventos de magnitudes maiores ou iguais a 5 , dois dos quais acima de 6.0 ocorridos no último século. Recentemente foi reportado um evento histórico ocorrido em 1690 na região do rio Amazonas com magnitude $\sim 7 \mathrm{Mw}$ : provavelmente o maior evento no ICE da placa Sul Americano (Veloso, 2014). Entretanto, nenhum desses sismos afetou severamente zonas urbanas povoadas. Apesar das baixas atenuações das ondas sísmicas no Brasil, que favorecerem intensidades elevadas para eventos de baixas magnitudes, as maiores intensidades observadas no Brasil foram VI VII na Escala Mercalli Modificada (MM). P.E. em Pacajus - CE, sismo de 20/11/1980 (Berrocal et al., 1984), João Câmara - RN, sismo de 30/11/1986 (Ferreira et al., 1987) e Codajás - AM, sismo de 05/08/1983 (Assumpção et al., 1983). Os eventos do NE produziram danos a construções de baixa qualidade e gerou um êxodo de moradores da zona rural de Joao Câmara e Pacajus. Recentemente, um sismo de magnitude 4.9, no meio do Craton do São Francisco, além de danos permanentes às construções da pequena Vila de Caraíbas - Itacarambi - MG, causou a única vítima fatal decorrente direta de terremoto no Brasil (Chimpliganond et al., 2010).

Os parâmetros sísmicos mais importantes de um terremoto são: localização hipocentral; magnitude ou tamanho; e mecanismo focal, que dá informações sobre a falha sismogênica: sua geometria, direção de movimento (escorregamento) e orientação dos esforços liberados na ruptura, o que permite inferências sobre o regime dos esforços na área epicentral.

Até recentemente, os estudos de mecanismos focais no Brasil são raros e difíceis de serem realizados devido à baixa sismicidade associada ao baixo número de estações sismográficas. Dessa forma, pouco se conhece sobre a 
distribuição dos esforços tectônicos na intraplaca brasileira, informação fundamental para o entendimento da sismicidade intraplaca e com aplicação em estudos de ameaça e risco sísmicos. Contudo, a implantação recente da Rede Sismográfica Brasileira (RSBR) (Bianchi et al., 2014; Pirchiner et al., 2011; Carvalho et al., 2009) irá facilitar esses estudos.

O conhecimento dos parâmetros sísmicos de fonte é pré-requisito para a compreensão de processos físicos que ocorrem durante os terremotos. Rotineiramente, são extraídas das formas de ondas dos registros sísmicos de terremotos informações contidas apenas na primeira chegada da onda $P$, a partir das quais se determina os parâmetros mencionados acima. Entretanto, muito mais pode ser inferido a respeito de aspectos cinemáticos e dinâmicos das fontes sísmicas quando se analisa o sismograma em sua totalidade ou em determinadas faixas de frequências. Normalmente, a utilização apenas da fase $P$ exige o registro do mesmo terremoto em várias estações. Já a análise mais detalhada das formas de ondas de um evento pode reduzir substancialmente 0 número de estações necessárias à determinação dos mesmos parâmetros.

Nesta dissertação de mestrado serão determinados parâmetros sísmicos de fontes de uma seleção de onze eventos pertencentes à sequência sísmica de Mara Rosa, GO, registrada por uma rede local de oito estações entre outubro de 2010 e abril de 2011. Esses sismos são réplicas do evento principal de 8 de outubro de $2010 \mathrm{com}$ magnitude $5.0 \mathrm{~m}_{\mathrm{b}}$ e intensidade VI (MM) (Barros et al. 2014). Também será apresentada a inversão do momento tensor para o evento principal de Mara Rosa.

As soluções de planos nodais para os microtremores foram obtidas a partir de informações contidas nas formas de ondas registradas por poucas estações da rede local, o que não seria possível utilizando o método tradicional de polaridades da onda P. Para o evento principal foram utilizados seus registros em três estações regionais e, em ambos os casos, foi utilizado inversão de formas de ondas com o programa ISOLA (Sokos \& Zahradnik, 2008, 2013); http://geo.mff.cuni.cz/ iz/isola brasilia/ 
Essa dissertação é apresentada na forma de artigo e é composta por cinco capítulos. No capítulo 1 é definido o problema e mostrada a importância de sua solução; no capitulo 2 é descrita a metodologia, com um estudo de fontes sísmicas, sua representação pelo momento tensor e o programa ISOLA; no capítulo 3 é apresentado o artigo"Focal mechanisms and moment magnitudes of micro-earthquakes in central Brazil by waveform inversion with quality assessment and inference of the local stress field" já submetido para o Journal of South American Earth Science, requisito parcial para obtenção do grau de mestre em Geociências Aplicadas; no capítulo 4 é apresentada a inversão do evento principal e; no capítulo 5 são apresentadas a discussão e conclusões da dissertação. No Anexo I é apresentada a produção científica (primeira página) produzida no âmbito do tema da dissertação com participação do autor e no Anexo II as formas de ondas (sintéticas e observadas) obtidas na inversão das onze réplicas

\subsection{Objetivos da dissertação}

\section{Objetivos gerais}

Determinar os parâmetros de fonte, por inversão de formas de ondas, de sismos locais e regional com epicentros na Zona Sísmica de Mara Rosa (ZSMR), utilizando o programa ISOLA - ISOLated Asperities (Sokos \& Zahradnik, 2008, 2013). As soluções obtidas foram avaliadas utilizando vários critérios de controle de qualidade para garantir resultados confiáveis nas inversões. Também é objetivo deste trabalho a obtenção da orientação do esforço local médio pela inversão dos vários mecanismos focais, com a utilização do programa STRESSINVERSE desenvolvido por Vavryčuk (2014).

\section{Objetivos específicos}

i. Determinar mecanismos focais para onze eventos de baixas magnitudes $(\mathrm{M}<2)$ com epicentros na ZSMR, por inversão de formas de ondas, utilizando o programa ISOLA com dados da rede sismográfica local.

ii. Determinar o mecanismo focal e a magnitude de momento sísmico do evento principal de Mara Rosa por inversão de formas de ondas com dados de estações regionais. 
iii. Determinar as magnitudes de momento sísmico (Mw) e compará-las com as magnitudes de duração das réplicas $\left(M_{D}\right)$ e magnitude regional $(\mathrm{mR})$ do evento principal.

iv. Comparar e discutir os parâmetros sísmicos obtidos neste trabalho com aqueles obtidos por Barros et al. (2014) com a utilização do método tradicional de polaridades.

v. Fazer inversão dos mecanismos focais dos microtremores com o software STRESSINVERSE desenvolvido por Vavryčuk (2014) para obter o esforço resultante na área.

\section{3. Área de estudo}

A área de estudo está localizada na faixa sísmica Goiás Tocantins que está inserida, quase integralmente, na Província Tocantins, conforme mostra a Figura 1.1.

A faixa sísmica Goiás Tocantins ou Goiás-Tocantins Seismic Zone (GTSZ), indicada pelo retângulo em amarelo na Figura 1.1, onde está inserida a área de estudo (retângulo branco), apresenta sismicidade distribuída em uma extensa faixa, com orientação NE-SW, paralela, mas não coincidente com o Lineamento Transbrasiliano (LTB) (Veloso et al., 1997). A faixa sísmica está localizada no Brasil central nos estados de Goiás, Tocantins e parte do Matogrosso; se estende desde o noroeste da Bacia do Paraná até o sudoeste da Bacia do Parnaíba e cuja expressão geofísica é caracterizada pelas altas anomalias gravimétricas junto à faixa de dobramento Tocantins Araguaia (Assumpção et al., 1986; Fernandes et al., 1991). A GTSZ é caracterizada por eventos de baixas magnitudes, a maioria com magnitudes menores que 3.5, sendo que nos últimos cinco anos foram registrados menos que cinco eventos com magnitudes acima de quatro.

O monitoramento instrumental da GTSZ iniciou em 1968 com a instalação do arranjo sismográfico SAAS (South America Array System) seguido da instalação da estação de banda larga WWSSN (Worldwide Standardized Seismograph Network) e, localmente após a década de 1990, com a implantação das redes sismográficas para o monitoramento dos reservatórios de Serra da Mesa, Cana Brava, São Salvador, Lajeado, Peixe e Estreito. Atualmente a região é monitorada por 12 estações sismográficas com 
transmissão em tempo real, sendo cinco da Rede Sismográfica do Observatório Sismológico da Universidade de Brasília, seis da Rede Sismográfica do Centro e Norte do Brasil, pertencente a Rede Sismográfica Brasileira (RSBR), e uma estação da rede global do CTBTO/IMS (International Monitoring System). Na região também existem estações de outros projetos e/ou instituições. O monitoramento da zona sísmica Goiás Tocantins que é caracterizada por eventos de baixas magnitudes não permite de forma confiável, a determinação dos momentos tensores dos pequenos tremores 0 que dificulta a determinação dos esforços na região.

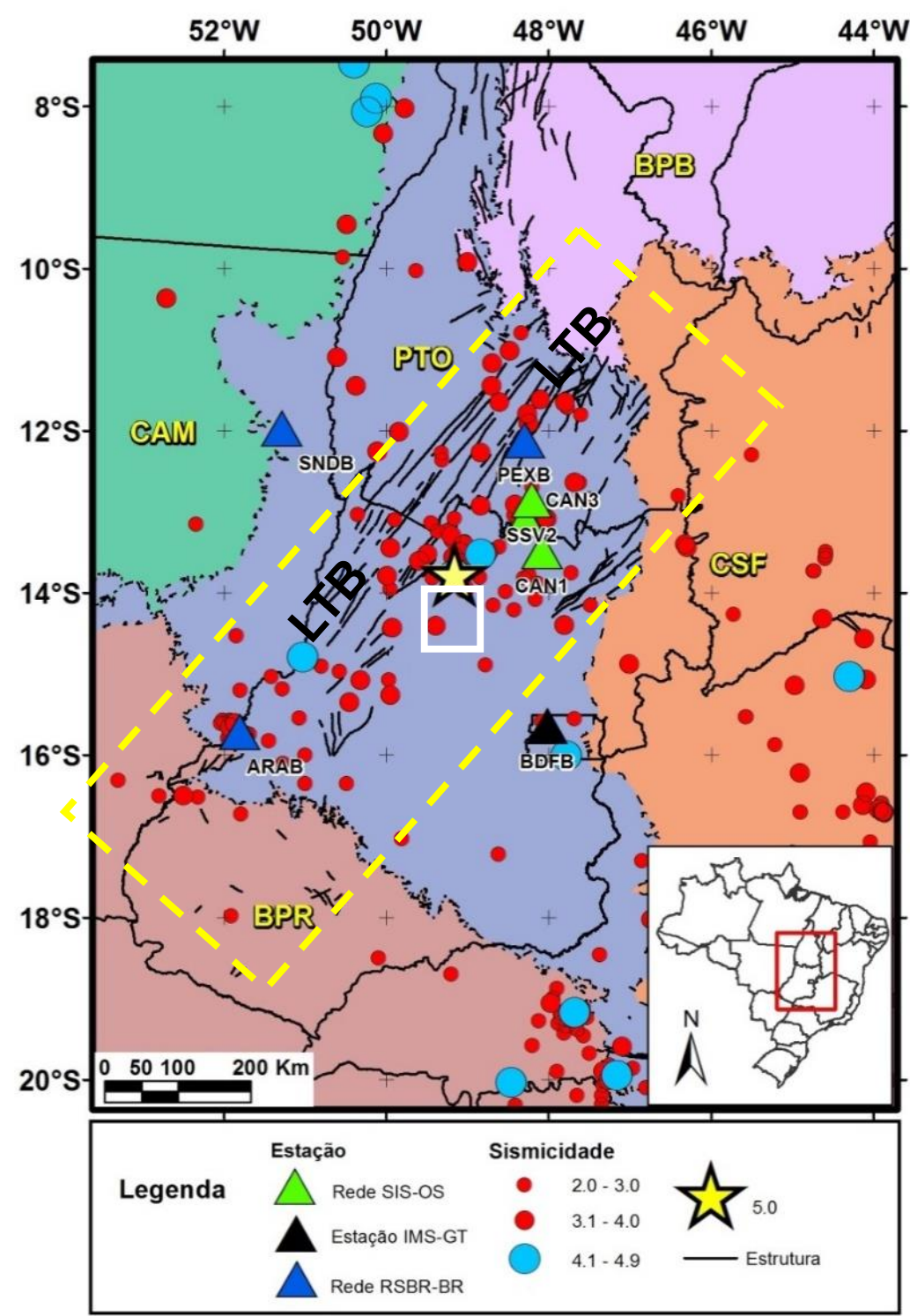

Figura 1.1 - Faixa sísmica Goiás-Tocantins (retângulo amarelo) e estruturas do Lineamento Transbrazilianos (LTB). O quadrado branco mostra a área de estudo e a estrela indica o evento principal de Mara Rosa. As províncias geológicas são: PTO (Província Tocantins); BPR (Bacia do Paraná); CAM (Craton Amazônoniano); BPB (Bacia do Parnaíba) e CSF (Craton São Francisco). 


\subsection{Tectônica regional}

O conhecimento da distribuição dos esforços crustais é fundamental para o entendimento da sismicidade, particularmente em regiões intraplaca (Zoback, 1992a \& 1992b). A maioria dos tensores de esforços em escala global foram determinados pela utilização de mecanismos focais (Heidbach et al., 2004).

O estado das tensões na intraplaca brasileira resulta da combinação de forças de origem regional e local. As forças locais são causadas por heterogeneidades estruturais resultantes de carregamento da crosta e anomalias térmicas na astenosfera. No caso especifico da faixa sísmica Goiás Tocantins, deformações flexurais (Assumpção \& Sacek, 2013) podem também influenciar na sismicidade. As de origem regional estão relacionadas com forças tectônicas com origem nas bordas das placas, tais como: empurrão da dorsal oceânica, produzindo esforço de compressão no interior da placa; forças de oposição produzidas pelas placas Nazca e do Caribe e empuxo negativo da placa de Nazca sobre a Sul Americana (Assumpção, 1992b).

Os primeiros estudos sobre mecanismo focal no Brasil que foram realizados por Mendiguren \& Richter (1978); Assumpção et al. (1997); Assumpção \& Suarez (1988); Assumpção (1992, 1998a, 1998b e 1998c) e Ferreira (1997). Recentemente, Barros et al. (2009); Chimpliganond et al. (2010); Barros (2010); Assumpção et al. (2014); Carvalho et al. (2014) e Barros et al. (2014) incorporaram aos dados anteriores novos resultados de estudos de mecanismos focais.

Como resultado desses estudos sabe-se que a intraplaca do Brasil está submetida a esforço de orientação médio $\mathrm{E}-\mathrm{W}$, exceção feita à parte norte, onde os esforços estão rotacionados para o norte (M. L. Zoback \& Richardson, 1996). A Figura 1.4 extraída de Assumpção et al. (2008) apresenta a distribuição do estado dos esforços na placa Sul Americana. 


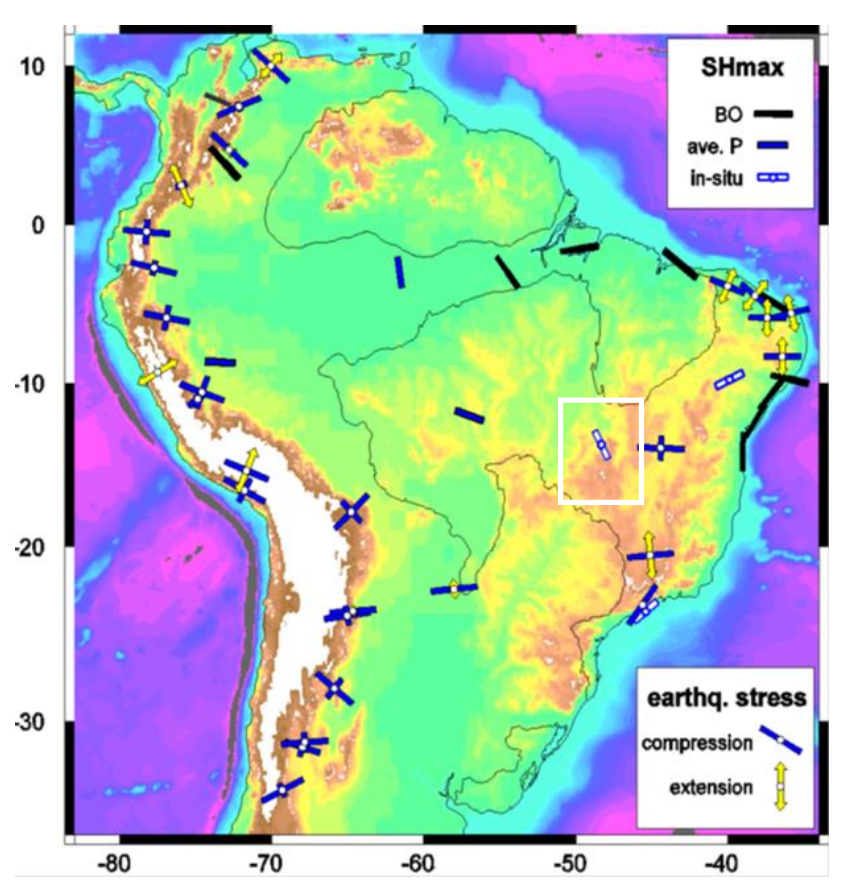

Figura 1.2 - Estimativa da direção dos esforços na placa Sul Americana a partir de dados de Mecanismos Focais, breakouts e medidas in-situ. As cores das barras representam: amarelo - tração e azul - compressão. Quadrado branco mostra a área de estudo. Figura de Assumpção et al. (2008).

\subsection{Dados utilizados}

Os dados usados nesta dissertação são as formas de ondas do evento principal registrado em três estações regionais da rede do SIS-OS (Figura 1.1), e formas de ondas de onze réplicas registradas pela rede local de oito estações (Figura 1.2). Ambas as redes são compostas por instrumentos de período curto e de banda larga. A rede local funcionou por 6 meses, entre outubro de 2010 e maio de 2011 e registrou mais de 600 pós abalos de pequenas magnitudes, dos quais 53 foram localizados com boa precisão (L. V. Barros, M. Assumpção, et al., 2014). A Tabela 1.1 apresenta as características dos instrumentos da rede sismográfica local e a Tabela 1.2 os instrumentos da rede regional. Os dados da rede local foram amostrados a uma taxa de $200 \mathrm{sps}$ e os da rede regional a 100 sps.

Os sismogramas em velocidade foram convertidos em deslocamento com a utilização dos arquivos de polos e zeros das funções de transferências dos sismógrafos, fornecidas pelo fabricante (Guralp Inc). 
Tabela 1.1 - Características dos instrumentos sismográficos utilizados na rede local de Mara Rosa. A estação MR5 foi remanejada para MR10. As estações funcionaram entre outubro de 2010 a maio de 2011.

\begin{tabular}{cccc}
\hline Estação & Equipamento & Banda passante & SN sensor \\
\hline MR03 & GURALP -6TD & $30 \mathrm{~S}-100 \mathrm{~Hz}$ & T6654/B304 \\
\hline MR04 & GURALP -6TD & $30 \mathrm{~S}-100 \mathrm{~Hz}$ & $T 6653 / B 306$ \\
\hline MR05 & GURALP -6TD & $30 \mathrm{~S}-100 \mathrm{~Hz}$ & $T 6655 / B 303$ \\
\hline MR07 & GURALP -6TD & $30 \mathrm{~S}-100 \mathrm{~Hz}$ & $T 6657 / B 307$ \\
\hline MR08 & GURALP -6TD & $30 \mathrm{~S}-100 \mathrm{~Hz}$ & $T 6652 / B 305$ \\
\hline MR09 & GURALP -6TD & $30 \mathrm{~S}-100 \mathrm{~Hz}$ & $T 6652 / B 305$ \\
\hline MR10 & GURALP -6TD & $30 \mathrm{~S}-100 \mathrm{~Hz}$ & $T 6655 / B 303$ \\
\hline MR11 & EAM/40T1 & $01-100 \mathrm{~Hz}$ & $T 4 Q 87 / A 1563$ \\
\hline MR12 & EAM/40T1 & $01-100 \mathrm{~Hz}$ & $A 1555 / T 4 Q 76$ \\
\hline
\end{tabular}

Tabela 1.2 - Características dos instrumentos sismográficos utilizados na rede regional de Mara Rosa. As estações funcionaram durante todo o período de monitoramento e continuam em operação.

\begin{tabular}{cccc}
\hline Estação & Equipamento & Banda passante & SN sensor \\
\hline CAN3 & $D M 24 / 40 T 1$ & $01-100 \mathrm{~Hz}$ & $B 826 / T 4 N 80$ \\
\hline SSV2 & $D M 24 / 40 T 1$ & $01-100 \mathrm{~Hz}$ & $B 827 / T 4 Q 92$ \\
\hline BDFB & EUROPA-T/CMG3TB & $120 \mathrm{~S}-100 \mathrm{~Hz}$ & $0739 / T 3 P 87$ \\
\hline
\end{tabular}




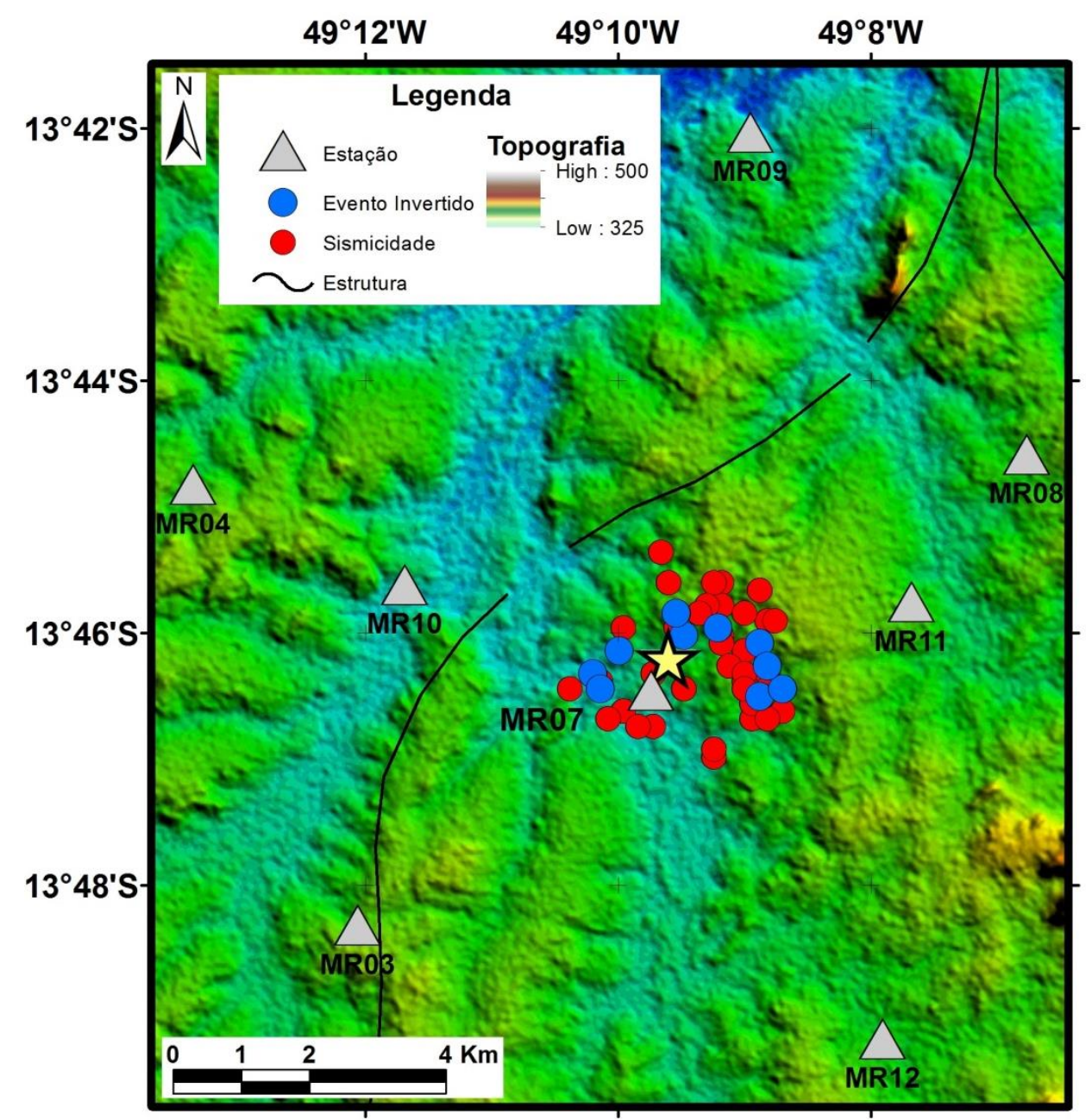

Figura 1.3 - Rede sismográfica local de Mara Rosa (triângulos) e epicentros dos eventos melhores registrados da atividade de réplicas (53, círculos vermelhos e azuis). Os 11 círculos azuis representam os eventos para os quais se realizou inversões. A estrela amarela denota o epicentro do evento principal, $5.0 \mathrm{~m}_{b}$ de 08/10/2010.

Do conjunto de 53 eventos foram selecionados onze (Tabela 1.3), registrados por quatro e até oito estações, com magnitudes variando entre 1,2 $m_{D} e$ 2,0 $\mathrm{m}_{\mathrm{D}}$ e distâncias epicentrais menores que $8,0 \mathrm{~km}$. As formas de ondas do evento 10 podem ser vistas na Figura 1.3. O modelo crustal utilizado na inversão foi derivado do trabalho de Soares et al. (2006), Figura 3.3 e Tabela 3.1. A relação Vp/Vs foi derivada de estudos de função receptor realizados por França \& Assumpção (2004). 
Tabela 1.3 - Lista de eventos selecionados para a inversão.

\begin{tabular}{|c|c|c|c|c|c|}
\hline \multicolumn{6}{|c|}{ ected for this stud } \\
\hline Event Number & Date (yyyy/mm/dd) & Origin Time (UTC) & Coordinate (DDMM) & Depth/Magnitude* & RMS \\
\hline 1 & $2011 / 03 / 30$ & $21: 40: 15.30$ & $-13^{\circ} 46.54-49^{\circ} 8.88$ & $0.8 / 1.4$ & 0.03 \\
\hline 2 & $2011 / 04 / 03$ & $09: 19: 49.19$ & $-13^{\circ} 46.11-49^{\circ} 8.87$ & $1.2 / 1.3$ & 0.03 \\
\hline 3 & $2011 / 04 / 03$ & $15: 51: 08.60$ & $-13^{\circ} 46.00-49^{\circ} 9.51$ & $1.4 / 1.3$ & 0.04 \\
\hline 4 & $2011 / 04 / 04$ & $10: 19: 46.61$ & $-13^{\circ} 45.87-49^{\circ} 9.54$ & $1.3 / 1.2$ & 0.04 \\
\hline 5 & 2011/04/09 & $19: 52: 13.16$ & $-13^{\circ} 45.96-49^{\circ} 9.21$ & $1.7 / 1.5$ & 0.03 \\
\hline 6 & $2011 / 04 / 15$ & $03: 02: 19.25$ & $-13^{\circ} 46.46-49^{\circ} 8.64$ & $0.6 / 1.3$ & 0.03 \\
\hline 7 & $2011 / 04 / 15$ & $03: 05: 35.15$ & $-13^{\circ} 46.46-49^{\circ} 8.64$ & $0.6 / 1.2$ & 0.03 \\
\hline 8 & $2011 / 04 / 16$ & $09: 01: 07.67$ & $-13^{\circ} 46.29-49^{\circ} 8.77$ & $1.0 / 1.2$ & 0.03 \\
\hline 9 & $2011 / 04 / 18$ & $06: 42: 20.83$ & $-13^{\circ} 46.34-49^{\circ} 10.17$ & $1.3 / 1.4$ & 0.02 \\
\hline 10 & $2011 / 04 / 18$ & $12: 45: 13.62$ & $-13^{\circ} 46.45-49^{\circ} 10.14$ & $1.2 / 2.0$ & 0.02 \\
\hline 11 & $2011 / 04 / 28$ & $13: 16: 36.23$ & $-13^{\circ} 46.14-49^{\circ} 9.99$ & $0.9 / 1.4$ & 0.02 \\
\hline
\end{tabular}

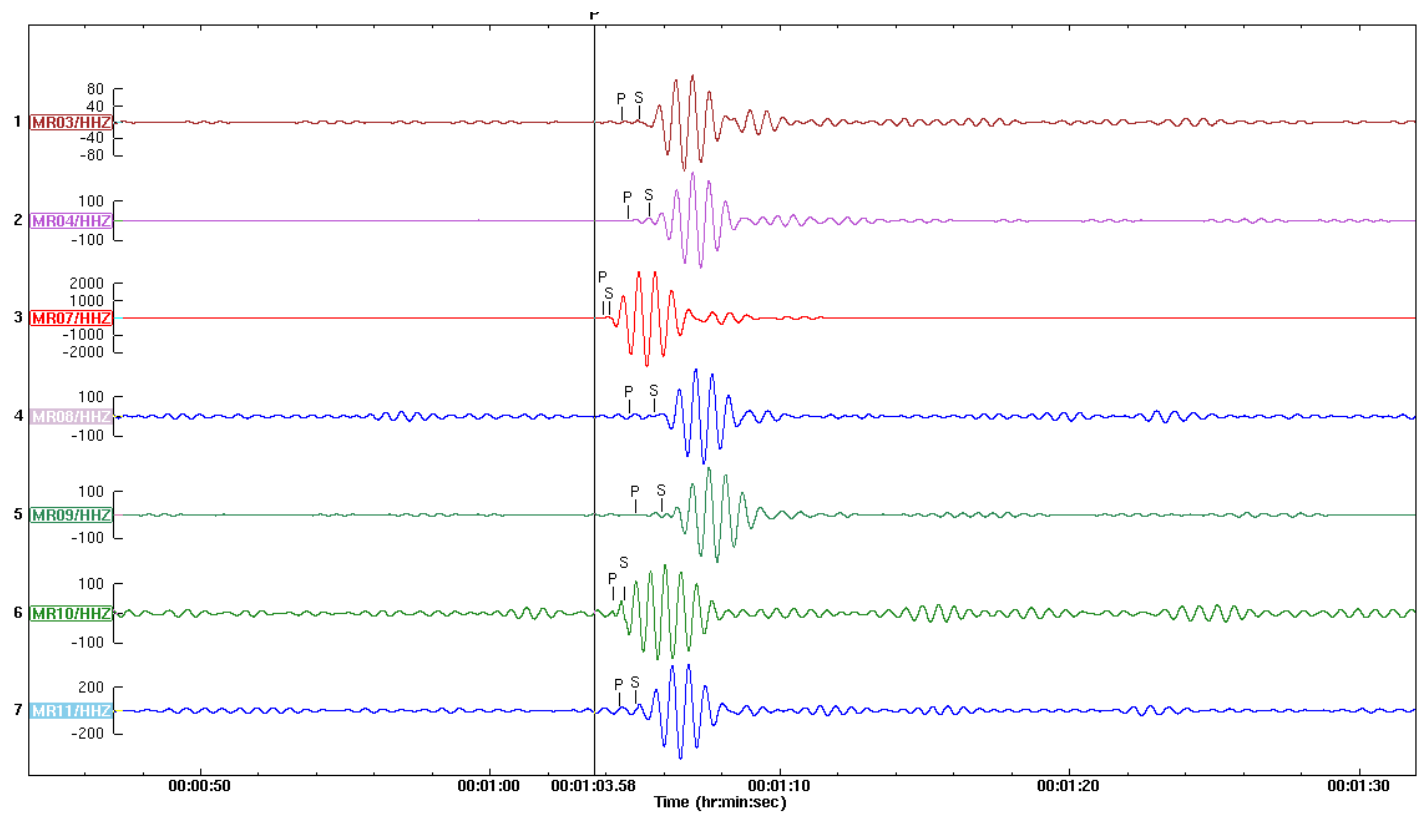

Figura 1.4 - Formas de ondas, nas componentes verticais, do evento 10 da Tabela 1.3 registrados pelas estações MR03, MR04, MR07, MR08, MR09, MR10 e MR11. Os dados são em velocidade (contagens digitais) e filtro 1.5 a $2.0 \mathrm{~Hz}$. O Traço vertical marca o tempo de origem (TO).

\subsection{Referências}

Assumpção, M. (1992). The Regional Intraplate Stress-Field in South-America. Journal of Geophysical Research-Solid Earth, 97, 11889-11903. Retrieved from <Go to ISI>://A1992JG86200013

Assumpção, M. (1998a). Focal mechanisms of small earthquakes in the southeastern Brazilian shield: a test of stress models of the South American plate. Geophysical Journal International, 133(2), 490-498. doi:10.1046/j.1365-246X.1998.00532.x

Assumpção, M. (1998b). Seismicity and stresses in the Brazilian passive margin. Bulletin of the Seismological Society of America, 88, 160-169. Retrieved from http://www.bssaonline.org/content/88/1/160.abstract

Assumpção, M. (1998c). Sismotectónica y esfuerzos en Brasil. In Física de la Tierra (Vol. 0). Retrieved from http://www.mendeley.com/research/sismotect?nica-y-esfuerzos-en-brasil/

Assumpção, M., Ortega, R., Berrocal, J., \& Veloso, J. A. V. (1983). O sismo de Codajás-AM. Revista Brasileira de Geofísica, 2, 39-44.

Assumpção, M., Lima, T. M., \& Tomás, L. A. (1986). O sismo de Araguapaz de 14.01 .1986 e o 
Lineamento Transbrasiliano. In 340 Congresso Brasileiro de Geologia, Goiânia (p. 5p).

Assumpção, M., \& Suarez, G. (1988). Source mechanisms of moderate-size earthquakes and stress orientation in mid-plate South America. Geophysical Journal International, 92(2), 253-267. doi:10.1111/j.1365-246X.1988.tb01138.x

Assumpção, M., Barbosa, J. R., Berrocal, J., Bassini, A. M., Veloso, J. A. V., Marza, V. I., ... Ribotta, L. C. (1997). Seismicity patterns and focal mechanisms in southeastern Brazil. Brasileira de Geofísica, 15, 119-132.

Assumpção, M., Lopes, A. E. V. E., Barros, L. V. V, Chimpliganond, C. N. N., \& Barbosa, J. R. R. (2008). Intraplate Stress Patterns in Brazil with Recent Focal Mechanism Studies: Predominance of Mid-Continent compressional stresses and flexural effects at the continental margin. In AGU Joint Assembly (pp. CDROM, 1p.). Fort Lauderdale.

Assumpção, M., \& Sacek, V. (2013). Intra-plate seismicity and flexural stresses in central Brazil. Geophysical Research Letters, 40(3), 487-491. doi:10.1002/grl.50142

Assumpção, M., Dias, F. L., \& Zevallos, I. (2014). Intraplate Stress Field in South America from Earthquake Focal Mechanisms. South American Earth Sci (Submited).

Barros, L. V., Assumpção, M., Quintero, R., \& Caixeta, D. (2009). The intraplate Porto dos Gaúchos seismic zone in the Amazon craton - Brazil. Tectonophysics, 469(1-4), 37-47. doi:10.1016/j.tecto.2009.01.006

Barros, L. V. (2010). Sismicidade, esforço tectônicos e estrutura crustal da zona sísmica de Porto dos Gaúchos - MT (Tese de doutorado).

Barros, L. V., M. Assumpção, Chimpliganond, C. N. C., Carvalho, J. M., Huelsen, M. G. Von, Caixeta, D. F., ... Albuquerque, D. F. (2014). The Mara Rosa 2010 GT-5 earthquake and its possible relationship with the continental-scale Transbrasiliano lineament,. J. S. Am. Earth Sci. (submetido).

Berrocal, J., Assumpção, M., ARTEZANA, R.DIAS NETO, C. M., Ortega, R., FRANÇA, H., VELOSO, J. A. V., ... Franca, H. (1984). Sismicidade do Brasil (p. 320p). Sao Paulo: Instituto Astronômico e Geofísico. Retrieved from http://www.mendeley.com/research/sismicidadebra-

sil/?utm_source=desktop\&utm_medium=1.12.3\&utm_campaign=open_catalog\&userDo cument/d $=\{103643 f 6-9736-4386-b 7 c 8-3 d c e 2 a 7691 c 1\}$

Bianchi, M., Assumpção, M., Agurto, H., Collaço, B., Calhau, J., Roberto, J., ... Barros, L. (2014). The New Brazilian Seismic Network: Current Status and Detectability Test with the Recent Pisagua Earthquake Sequence, Northern Chile. Earth Sci. Res. J, 18(Special Issue), 45.

Carvalho, J. M., L., B., \& Franca, G. S. (2009). A new seismograph network to improve the seismic monitoring in Brazil. In: IASPEI 2009 - South Africa. In IASPEI 2009. Cidade do Cabo, South Africa.

Carvalho, J. M., Barros, L., \& Zahradnik, J. (2014). Focal mechanisms and moment magnitudes of micro- earthquakes in central Brazil by waveform inversion with quality assessment and inference of the local stress field. Journal of South American Earth Sciences (submitted). 
Chimpliganond, C., Assumpção, M., Von Huelsen, M., \& França, G. S. (2010). The intracratonic Caraíbas-Itacarambi earthquake of December 09, 2007 (4.9 mb), Minas Gerais State, Brazil. Tectonophysics, 480(1-4), 48-56. doi:10.1016/j.tecto.2009.09.016

Fernandes, E. P., Blum, M. L. A., \& Ribeiro, R. (1991). The Goiás seismic zone, a new approach. In Extended Abstract. $35^{\circ}$ Congress Braz. Geol. Soc., Salvador/BA (Vol. 2, pp. 553-558).

Ferreira, J., Takeya, M., Costa, J. M., Moreira, J. A., Assumpção, M., Veloso, J. A. V., \& Pearce, R. G. (1987). A continuing intraplate earthquake sequence near João Câmara, Northeastern Brazil, preliminary results. Geophys. Res. Lett, 14, 1042-1045.

Ferreira, J. (1997). Sismicidade e esforços no Nordeste do Brasil (Dissertação de mestrado). IAGUSP.

França, G. S. (2004). Estrutura da crosta no sudeste e centro-oeste do Brasil, usando função do receptor local: São Paulo. Revista Brasileira de Geofísica, 22(2), 143f. doi:10.1590/S0102261X2004000200007

França, G. S., \& Assumpção, M. (2004). Crustal structure of the Ribeira fold belt, SE Brazil, derived from receiver functions. Journal of South American Earth Sciences, 16(8), 743-758. doi:10.1016/j.jsames.2003.12.002

Heidbach, O., Barth, A., Connolly, P., Fuchs, K., Müller, B., Tingay, M., ... Wenzel, F. (2004). Stress maps in a minute: The 2004 world stress map release. Eos, Transactions American Geophysical Union, 85(49), 521. doi:10.1029/2004EO490001

Mendiguren, J. A., \& Richter, F. M. (1978). On the origin of compressional intraplate stresses in South America. Physics of the Earth and Planetary Interiors, 16(4), 318-326. doi:10.1016/0031-9201(78)90070-5

Pirchiner, M., \& Collaço, B. (2011). The BRAzilian Seismographic Integrated Systems (BRASIS): infrastructure and data management. Annals of Geophysics. doi:10.4401/ag-4865

Soares, J., Berrocal, J., Fuck, R. A., Mooney, W., \& Ventura, D. B. R. (2006). Seismic characteristics of central Brazil crust and upper mantle: A deep seismic refraction study. Journal of Geophysical Research, 111(B12), B12302. doi:10.1029/2005JB003769

Sokos, E. N., \& Zahradnik, J. (2008). ISOLA a Fortran code and a Matlab GUI to perform multiple-point source inversion of seismic data. Computers \& Geosciences, 34(8), 967-977. doi:10.1016/j.cageo.2007.07.005

Sokos, E., \& Zahradnik, J. (2013). Evaluating Centroid-Moment-Tensor Uncertainty in the New Version of ISOLA Software. Seismological Research Letters, 84(4), 656-665. doi:10.1785/0220130002

Vavryčuk, V. (2014). Iterative joint inversion for stress and fault orientations from focal mechanisms. Geophysical Journal International, 199(1), 69-77. doi:10.1093/gji/ggu224

Veloso, J. A. V., Marza, V. I., Carvalho, J. M., \& Barros, L. V. (1997). Recent seismic activity in the $S-W$ edge of the Mato Grosso-Goiás-Tocantins (MGT) Belt (Central Brazil). In 5th International Congress of the Brazilian Geophysical Society (pp. V2 - 964-967). Sao Paulo.

Veloso, J. A. V. (2014). On the footprints of a major Brazilian Amazon earthquake. Anais Da 
Academia Brasileira de Ciências, 86(3), 1115-1129. doi:10.1590/00013765201420130340

Zoback, M. Lou. (1992a). First- and second-order patterns of stress in the lithosphere: The World Stress Map Project. Journal of Geophysical Research, 97(B8), 11703. doi:10.1029/92JB00132

Zoback, M. Lou. (1992b). Stress field constraints on intraplate seismicity in eastern North America. Journal of Geophysical Research, 97(B8), 11761. doi:10.1029/92JB00221

Zoback, M. L., \& Richardson, R. M. (1996). Stress perturbation associated with the Amazonas and other ancient continental rifts. J. Geophys. Res., B, Solid Earth Planets, 101(3), 54595475. 


\section{CAPÍTULO 2 - METOLOGIA}

\subsection{Introdução}

Os parâmetros de fonte de um terremoto podem ser obtidos pela inversão de suas formas de ondas em determinadas bandas de frequências. A inversão é realizada em um processo interativo, com a comparação dos sismogramas observado e sintético, para obter o resultado de melhor ajuste. Um sismograma observado $u(t)$ é função da fonte $x(t)$, do meio $g(t)$ e da resposta do instrumento $i(t)$, Figura 2.1a. Dessa forma, a fonte $x(t)$, pode ser obtida a partir do sismograma observado $u(t)$, desde que sejam conhecidos $o$ meio, $g(t)$ e a resposta do instrumento, $i(t)$.

$$
u(t)=x(t) * g(t) * i(t)
$$

O símbolo $\left(^{*}\right)$ representa a operação de convolução, que, no domínio da frequência, equivale a uma multiplicação, ou seja:

$$
U(\omega)=X(\omega) \cdot G(\omega) \cdot I(\omega)
$$

Onde $\quad U(\omega), X(\omega), G(\omega)$ eI $(\omega)$ representam, respectivamente, as transformadas de Fourier de $u(t), x(t), g(t)$ e $i(t)$, conforme Figura 2.1b).

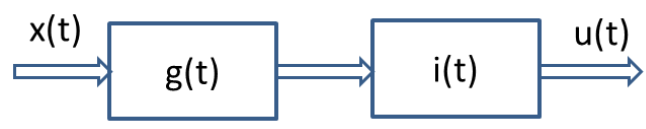

1a)

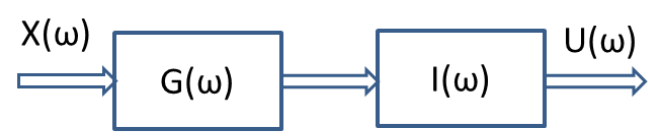

1b)

Figura 2.1 - Diagrama em blocos do sistema representando a fonte, $x(t)$, o meio, $g(t)$ e o instrumento, $i(t)$, no domínio do tempo (a) e no domínio da frequência (b). 


\subsection{Parâmetros de fonte}

Os parâmetros que definem uma fonte sísmica são o strike $(\varphi)$, o dip $(\delta)$, e o rake ( $\lambda$ ) e o momento sísmico escalar $\left(M_{0}\right)$. O desenvolvimento de um modelo para representar adequadamente uma fonte sísmica levou mais de 40 anos, desde os primeiros trabalhos de Nakano (1923), utilizando um modelo de esforço do tipo par-de-forças (single-couple), até os primeiros trabalhos com modelos de esforço duplo par-de-forças (double-couple) e fontes finitas desenvolvidos somente no início da década de 1960.

A teoria fundamental de fonte sísmica foi estabelecida nos trabalhos independentes de Maruyama (1963) e Burridge \& Knopoff (1964), os quais concluíram que uma falha cisalhante em meio elástico e isotrópico é equivalente a uma fonte do tipo duplo-par-de-forças (double-couple), Figura 2.2.

Single couples with torque

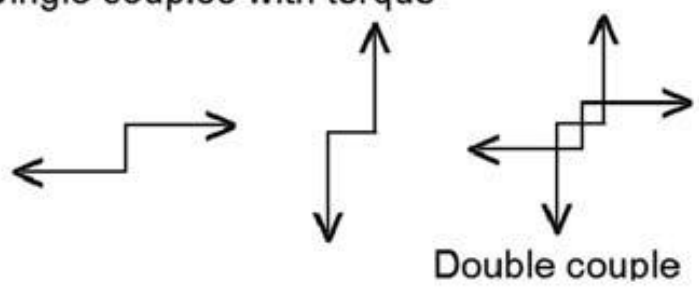
Singel couples without torque

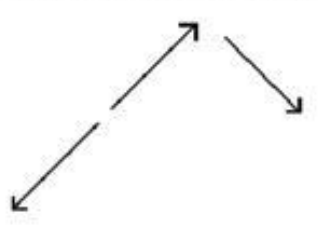

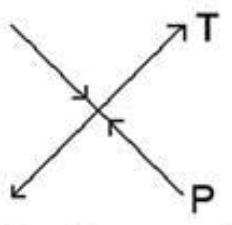

Double couple

Figura 2.2 - Tipos de forças que podem gerar deslocamento observados a longas distâncias (far field), provenientes da ruptura de uma falha (Havskov \& Ottemöller, 2010).

O modelo adotado na solução dos planos de falhas é equivalente a um duplo par-de-forças (double-couple) que pode ser descrito matematicamente como a soma de dois single-couples. No entanto, em condições reais, uma fonte sísmica pode ser mais complexa que a representada por apenas um doublecouple.

A irradiação da energia a partir da fonte sísmica pode ser descrita como a combinação de nove pares-de-forças. Três deles são dipolos ao longo dos eixos de coordenadas e os outros 6 pares de forças são do tipo single-couples, que, se agrupadas formam 3 double-couples, um em cada plano de coordenadas, Figura 2.3. Por conveniência matemática, os nove pares de forças são agrupados em uma matriz $3 \times 3$, denominada Momento Tensor $(M)$, equação 3. 

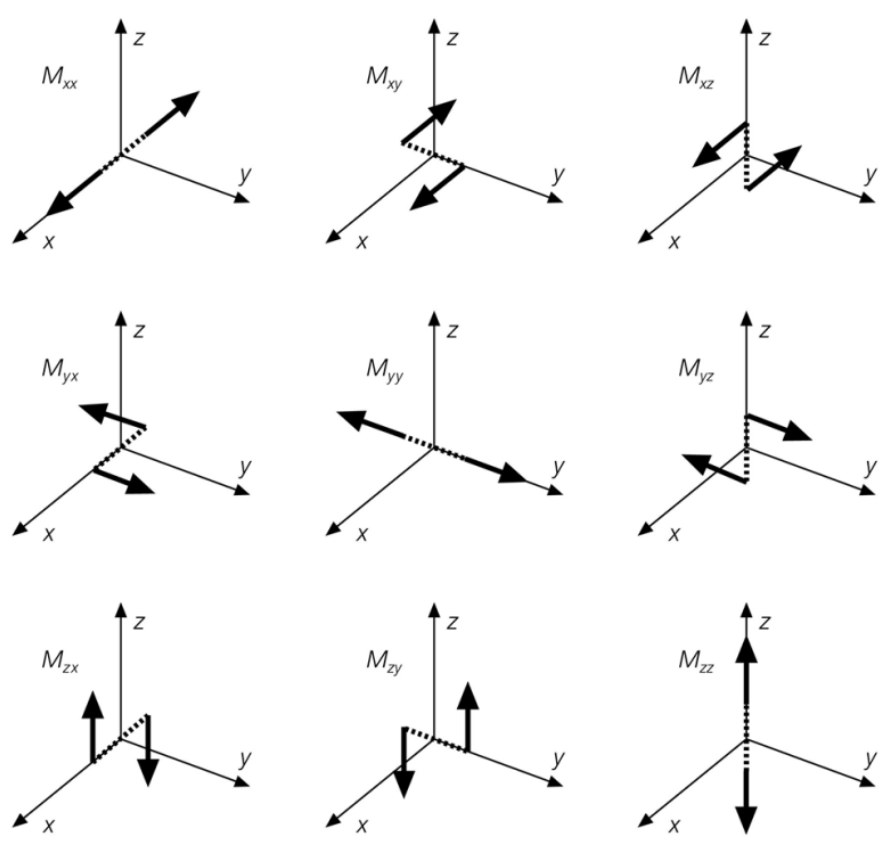

Figura 2.3 - Nove pares de forças que representam o momento tensor. E.g., Mxy é uma força dupla no plano xy agindo na direção do eixo $\boldsymbol{x}$ (Stein \& Wysession, 2003).

$$
M=\left[\begin{array}{lll}
M x x & M x y & M x z \\
M y x & M y y & M y z \\
M z x & M z y & M z z
\end{array}\right]
$$

O mecanismo focal descreve a deformação inelástica (irreversível) ocorrida em uma área durante um terremoto, ou seja, descreve a geometria da faIha e movimentação relativa dos blocos falhados, Figura 2.4. Os parâmetros que definem uma falha geológica de acordo com Aki \& Richards (2002), são: o strike $(\varphi)$, a orientação da falha em relação ao norte geográfico $\left(0^{\circ}\right.$ a $\left.360^{\circ}\right)$; 0 dip ( $\boldsymbol{\delta}$ ) ou mergulho, ângulo do plano da falha a partir da superfície $\left(0^{\circ}\right.$ a $\left.90^{\circ}\right) \mathrm{e}$ o rake (ג) ou ângulo do vetor deslocamento (slip), que é o ângulo entre a direção da falha e a direção do escorregamento do bloco superior, denominado de teto $\left(-180^{\circ}\right.$ a $\left.180^{\circ}\right)$.

Além dos parâmetros strike, dip e rake o estudo de mecanismo focal fornece ainda a orientação dos eixos de compressão $(P)$ e distensão $(T)$, responsáveis pela ruptura ou deslizamento da falha.

O mecanismo focal é, portanto derivado da solução do momento tensor através da análise das formas de ondas observadas em diversas estações sismográficas. 


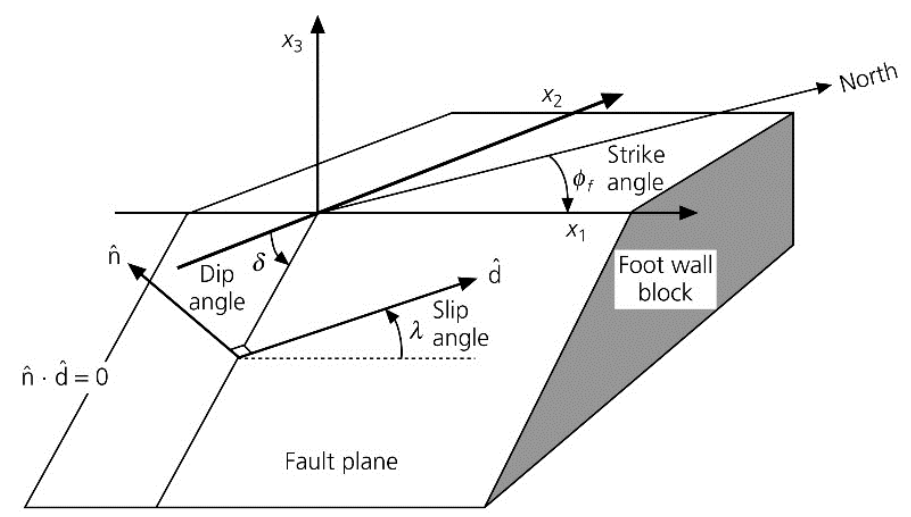

Figura 2.4 - Parâmetros de uma falha. A orientação do plano da falha é definida pelos ângulos dip e strike. O movimento relativo dos blocos é definido pelo ângulo do vetor slip (rake) (Stein \& Wysession, 2003).

A esfera focal mostra a projeção dos raios irradiados no hemisfério inferior, a partir da fonte nos ponto de observação, Figura 2.5a. Os parâmetros de uma falha podem ser representados com um diagrama do tipo estereográfico conhecido como beachball ou bola de praia. O beachball é a projeção da esfera focal na superfície, Figura 2.5b. Os quadrantes do "beachball são determinados a partir da orientação da falha e dos eixos de compressão $(P)$ e distensão $(T)$.

As partes hachuradas em preto na Figura 2.5b são áreas de compressão. O deslocamento do chão, registrado na estação sismográfica correspondente a esse raio, tem a primeira fase $(P)$ para cima, e as partes em branco são áreas de tração e o respectivo deslocamento tem a primeira fase $(P)$ para baixo.

a)

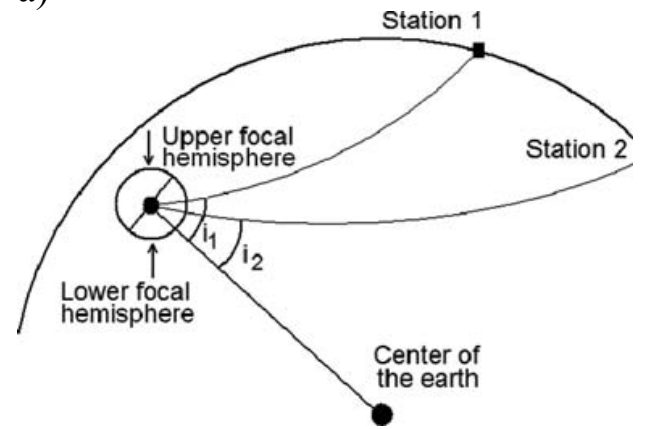

b)

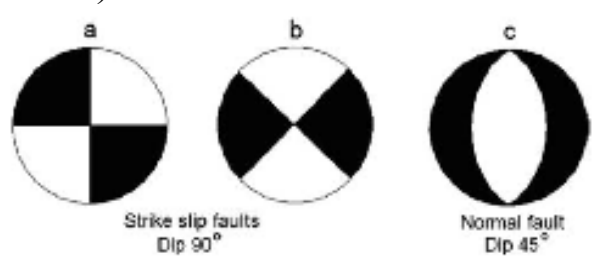

Figura 2.5 - O diagrama à esquerda mostra a esfera focal e a projeção dos raios sísmicos em direção às estações. O diagrama da direita mostra os beachballs das falhas do tipo strike slip e normal. Os quadrantes hachurados, em preto e branco, correspondem às áreas de compressão e tensão, respectivamente (Havskov \& Ottemöller, 2010).

A Figura 2.6 é uma representação dos diversos tipos de falhas e respectivas bolas de praia. 


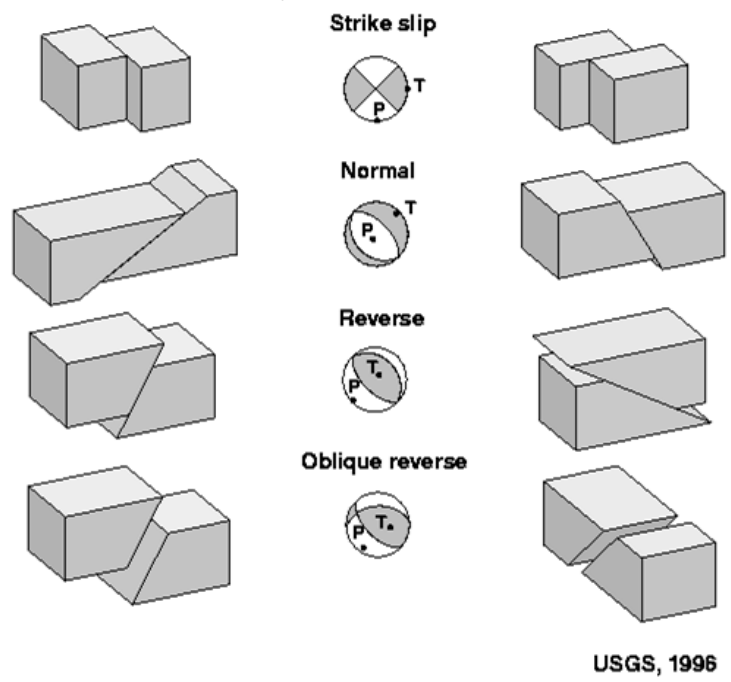

Figura 2.6 - Tipos de falhas e respectivos esforços (P\&T) necessários para sua ruptura (http://earthquake.usgs.gov/learn/topics/beachball.php).

A assinatura do sinal no sismograma é definida inicialmente na fonte pela orientação geográfica da falha e pela movimentação relativa dos blocos falhados, durante o processo de ruptura responsável pela ocorrência de um terremoto (Lay \& Wallace, 1995). As amplitudes das fases P e S dependem da direção de irradiação dos raios sísmicos da fonte (Figura 2.7). Os métodos mais utilizados na determinação de mecanismos focais são baseados nas polaridades das fases $\mathrm{P}$ e SH registradas nas diversas estações sismográficas e na razão das amplitudes das fases $\mathrm{P}, \mathrm{SV}$ e SH. Entretanto, esses métodos exigem o conhecimento das polaridades em muitas estações, nem sempre disponíveis. Além disso, em regiões de baixa sismicidade, é difícil conseguir eventos com polaridades suficientes. 

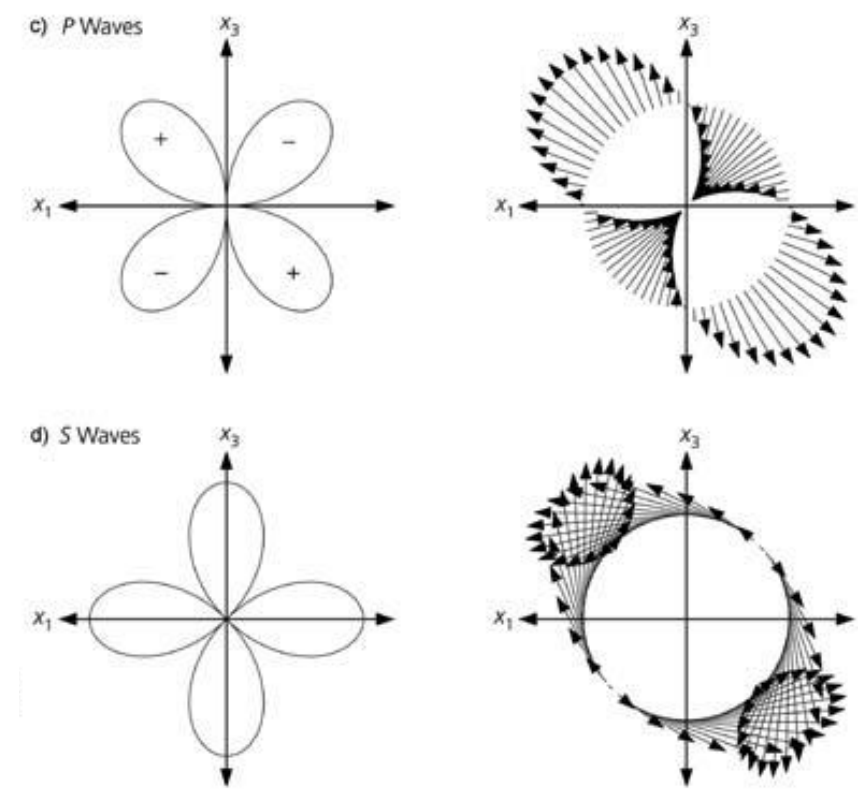

Figura 2.7 - Padrão de irradiação (esquerdo amplitudes e direito direção) das ondas $P$ (a) e $S(b)$, considerando uma fonte pontual do tipo Double Couple (Stein \& Wysession, 2003).

A obtenção de mecanismo focal pela inversão de formas de ondas de terremotos médios e grandes já é uma rotina em diversos Observatórios Sismológicos (Jiménez, Cara, \& Rouland, 1989; Lay \& Wallace, 1995). Entretanto, em relação aos eventos de baixas magnitudes, é mais difícil e raro (Fan \& Wallace, 1991; Benetatos et al., 2012; Fojtíková et al., 2010). Embora não seja ainda rotina, dados de eventos pequenos registrados a distâncias locais já podem ser invertidos utilizando alguns programas computacionais específicos e já disponíveis (Dreger \& Helmberger, 1993). Nesse trabalho utilizaremos o programa ISOLA desenvolvido por Sokos \& Zahradnik (2008, 2013).

\subsection{O programa ISOLA}

A inversão de formas de ondas com o programa ISOLA é fundamentada na representação de fontes pontuais e multiponto, usando método de deconvolução interativa semelhante ao método de Kikuchi \& Kanamori (1991). O ISOLA usa as formas de ondas (full wavefield) e filtragem sem fazer distinção de fases. A determinação do mecanismo focal para fontes pontuais é baseada no cálculo dos mínimos quadrados do momento tensor e busca da posição/tempo do centroide com melhor ajuste. As funções de Green são calculadas com o método do número de ondas discreto de Bouchon (1981) 
para um modelo 1D, tornando o método aplicável para inversão de formas de ondas de eventos registrados em distâncias locais e regionais.

\section{Metodologia do programa ISOLA}

Considerando as formas de ondas de uma fonte pontual em uma determinada posição e tempo de origem, o deslocamento $u$ pode ser expresso pelo momento tensor M e a derivada espacial do tensor de Green $G$ (Aki \& Richards, 2002):

$$
u_{i}(t)=\sum_{p=1}^{3} \sum_{q=1}^{3} M_{p q} * G_{i p, q}
$$

Onde * representa convolução temporal, e p, q as três coordenadas cartesianas.

O momento tensor pode ser expresso pela combinação linear dos seis tensores elementares adimensionais $\mathbf{M}^{\mathbf{i}}$ (Figura 2.8).

$$
M_{p q}=\sum_{i=1}^{6} a_{i} M_{p q}^{i}
$$

Desta forma, a fonte é caracterizada, convenientemente, pelos seis coeficientes escalares $a_{i}$.

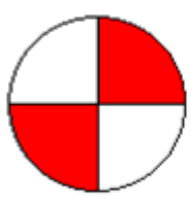

M1

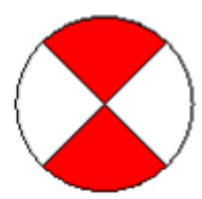

M2

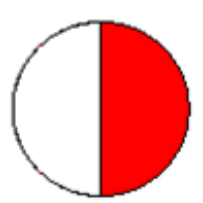

M3

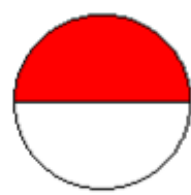

M4

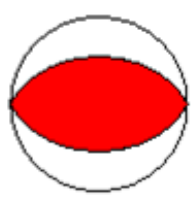

M5

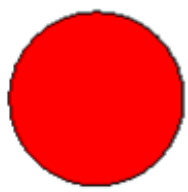

M6

Figura 2.8 - Momentos tensores elementares usados na inversão do momento tensor completo (Kikuchi \& Kanamori, 1991).

São usados os tensores elementares, implementado programa de simulação numérica (Bouchon, 1981; Coutant, 1990). Os tensores $\mathbf{M}^{\mathbf{1}}$ a $\mathbf{M}^{\mathbf{5}}$ representam as cinco variações básicas dos mecanismos focais do tipo double-couple (DC), 
enquanto $\mathbf{M}^{6}$ representa uma fonte puramente isotrópica. Combinando (5) com os tensores elementares; tem se:

$$
\boldsymbol{M}=\left[\begin{array}{ccc}
-a_{4}+a_{6} & a_{1} & a_{2} \\
a_{1} & -a_{5}+a_{6} & -a_{3} \\
a_{2} & -a_{3} & a_{4}+a_{5}+a_{6}
\end{array}\right]
$$

Onde os $a_{i}$ (dimensão do momento) são coeficientes da combinação linear da equação (5). Podemos deduzir que o traço do momento tensor, $\operatorname{tr}(\boldsymbol{M})=3 a_{6}$. O momento sísmico escalar é definido como a norma Euclidiana do MT (Silver \& Jordan, 1982).

$$
M_{o}=\sqrt{\left(\sum_{p=1}^{3} \sum_{q=1}^{3}\left(M_{p q}\right)^{2}\right)} / 2
$$

Combinando (4) e (5) temos:

$$
u_{i}(t)=\sum_{j-1}^{6} a_{j} E_{i}^{j}(t)
$$

Onde $E^{j}$ denota o sismograma elementar j-enésimo correspondendo ao momento tensor elementar j-enésimo. Aqui é assumido que a função momento é conhecida.

$\mathrm{Na}$ forma de matriz o sismograma $\boldsymbol{u}$ pode ser expresso como:

$$
\boldsymbol{u}=\boldsymbol{E} \boldsymbol{a}
$$

O programa usa informação da velocidade do chão registrada por instrumentos de banda larga e de período curto. Um filtro banda passante é aplicado para selecionar a faixa de frequência de interesse.

O sismograma observado (em velocidade) é inicialmente transformado em deslocamento. A inversão é feita em deslocamento e gera sismogramas 
sintéticos $s(t)$ formados pela combinação dos seis sismogramas elementares, $e_{i}(t), i=1,2, \ldots 6$, e.g., $\boldsymbol{s}(\boldsymbol{t})=\sum\left(\boldsymbol{a}_{\boldsymbol{i}} \cdot \boldsymbol{e}_{\boldsymbol{i}}(\boldsymbol{t})\right)$, onde os sismogramas elementares correspondem aos mecanismos focais básicos mostrados na Figura 2.8.

Os sismogramas triaxiais de um conjunto de estações representam os dados, $\boldsymbol{u}$, enquanto os coeficientes $\boldsymbol{a}_{\boldsymbol{i}}$ são os parâmetros a serem determinados (a). O problema linear inverso $\boldsymbol{u}=\boldsymbol{E} \boldsymbol{a}$ é resolvido pelo método dos mínimos quadrados, com $\boldsymbol{a}=\left(\boldsymbol{G}^{\boldsymbol{T}} \cdot \boldsymbol{G}\right)^{-\mathbf{1}} \boldsymbol{G}^{\boldsymbol{T}} \boldsymbol{u}$, onde $\boldsymbol{G}^{\boldsymbol{T}}$ é a transposta de $\boldsymbol{G}$ e ()$^{-1}$ é o inverso da matriz sistema $\left(\boldsymbol{G}^{\boldsymbol{T}} \cdot \boldsymbol{G}\right)$. Os autovalores da matriz sistema $\left(\boldsymbol{G}^{T} . \boldsymbol{G}\right)$ são calculados para estabelecer se o problema inverso tem bom ajuste, i.e., não é singular. $O$ valor da razão entre mínimo e o máximo autovalor da matriz (Condition Number-CN) é reportado como parâmetro de validação da inversão.

No caso da posição e tempo de origem não serem conhecidos, esses parâmetros adicionais (posição do centroid e tempo, contidos em G) seriam buscados (em loop) com o auxilio de uma grade de busca (grid search) para diferentes $\boldsymbol{G}$.

A grade de busca tem como objetivo encontrar a máxima correlação (Corr) entre os sismogramas observados (u) e o sintético (s).

$$
\operatorname{Corr}=\frac{\int u s}{\sqrt{\int u^{2} \int s^{2}}}
$$

Onde $\int u s$ (Eq. 11) é a somatória de todas estações e componentes.

$$
\int u s=\sum_{i} \int u s(t) S_{i}(t) d t
$$

A medida do desajuste (misfit) entre o sismograma real e o sismograma de melhor ajuste é obtida pela norma-L2

$$
\text { misfit }=\int(u-s)^{2}
$$


e/ou pela média global da redução de variância (VR).

$$
V R=1-\frac{m i s f i t}{\int u^{2}}
$$

Se o sintético for encontrado pela minimização misfit pelo método dos mínimos quadrados, $s=E a_{\text {opt. }}$ Nesse caso, a relação entre a correlação e a redução de variância $(V R)$ é dada por:

$$
\operatorname{Corr}^{2}=\mathrm{VR}
$$

\section{Decomposição do Momento Tensor}

O momento tensor pode ser decomposto em componentes Isotrópica (ISO), Double Couple (DC) e Compensated Linear Vector Dipole (CLVD).

Existem várias maneiras de interpretar o momento tensor resultante, i.e., a decomposição do MT em componentes. Seguindo a decomposição tradicional, $M=M_{I S O}+M_{D E V I A}$, onde $M_{I S O}$ e $M_{D E V I A}$ são as partes isotrópicas e desviatórica, respectivamente. Ademais, $M_{D E V I A}=M_{D C}+M_{C L V D}$ (e.g., Julian, 1998 , p. 530). Os autovalores do $M_{\text {DEVIA }}$ definem o parâmetro comum usado $\varepsilon$, variando de 0 (puro DC) a +/- 0.5 (puro CLVD): $\varepsilon=-\mathrm{e}_{1} / \mathrm{abs}\left(\mathrm{e}_{2}\right)$, onde $\mathrm{e}_{1}$ e $\mathrm{e}_{2}$ são os autovalores do $M_{D E V I A}$ com os valores absolutos mínimo e o máximo, respectivamente. $O$ tamanho relativo das componentes individuais é em porcentagem. Suas definições não são padronizadas na literatura. Neste trabalho, foi adotada a porcentagem definida na equação (8) de Vavryčuk (2001): ISO = $100[\operatorname{tr}(\mathrm{M}) / 3] / \mathrm{abs}\left(\mathrm{e}^{*}\right)$, onde $\mathrm{e}^{*}$ é o autovalor do momento tensor (full) $\mathrm{M}$, ○ qual tem o máximo valor absoluto, CLVD $=2 \varepsilon[100-\operatorname{abs}($ ISO)], $D C=100-a b s(I S O)-a b s(C L V D)$. Alternativamente neste trabalho, a parte isotrópica do MT também é medida diretamente por intermédio do coeficiente $\mathrm{a}_{6}$ de acordo com $a_{6}=\operatorname{tr}(\boldsymbol{M}) / 3$

\section{Aplicação}

O programa ISOLA vem sendo aplicado com sucesso no estudo de uma variedade grande de fontes sísmicas, a saber: Inversão de ondas para ob- 
tenção do momento tensor de eventos de magnitudes $\sim 4$, com exemplos de eventos ocorridos no golfo Corinthian, Grécia (Zahradník et al., 2008); determinação de mecanismo focal de micro tremores na área sísmica de Dobra Voda, Slovakia (Fojtíková et al., 2010); na inversão de dados sísmicos de fontes multipontos (E. N. Sokos \& Zahradnik, 2008); na deconvolução iterativa de formas de ondas de eventos regionais e interpretação de evento duplo do terremoto Lefkada, Grécia, 2003 (Zahradnik, Serpetsidaki, Sokos, \& Tselentis, 2005a); na avaliação das incertezas na determinação do centroide momento tensor ( $E$. Sokos \& Zahradnik, 2013); na avaliação da qualidade dos dados e correção das formas de ondas contaminadas por pulsos de longa duração (Zahradnik \& Plesinger, 2005), bem como na avaliaçao da solução do momento tensor quando aplicado ao estudo de sismos na penísula lbéricas, Zahradnik \& Custodio (2012); e, por fim, no estudo e caracterização de fontes sismicas explosivas associadas ao vulcão Columbo, Grécia (Heidbach et al., 2004; Krizova et al., 2013).

\subsection{Inversão de dados de mecanismos focais}

Como já visto, o estudo de mecanismo focal de um terremoto permite caracterizar a falha no que diz respeito à orientação do plano de ruptura, direção do mergulho e direção de seu movimento. Cada parâmetro desses é representado por um ângulo, determinado por observações nos registros sísmicos, considerando que nos sismogramas estão contidas informações sobre a natureza da falha e regime de falhamento, dado pelas direções dos eixos de compressão $(P)$ e de distensão $(T)$, obtidos no estudo de mecanismo focal. Essas são as direções dos esforços responsáveis pela ruptura de uma única falha e apenas no instante de sua ruptura (Michael, 1987a).

Ocorre que os sismos acontecem em diferentes falhas, com diferentes localizações e em diferentes tempos. Neste sentido, existe um conjunto de esforços distribuídos com diferentes orientações em uma dada região sismogênica, definindo, assim, um campo de esforços (stress field) na região. No caso dos mecanismos focais dos 11 microtremores de MR como será visto adiante, cada um é representado por um momento tensor, já que foram originados em 11 falhas diferentes, mobilizadas em diferentes tempos. 
A inversão de mecanismos focais, na obtenção da distribuição dos esforços principais em uma dada área, é uma das poucas ferramentas disponíveis para determinar as direções dos esforços principais das profundidades sismogênicas (Maury et al., 2013). Vários métodos têm sido propostos para a realização de tais inversões (Angelier, 2002).

Inversões de esforços determinados com estudos de mecanismos focais exigem o conhecimento de qual plano nodal é o plano de falha. Se essa informação não existir, e o plano de falha for trocado pelo plano auxiliar, as inversões de esforços podem produzir resultados equivocados. É Mostrado que o método de inversão linear desenvolvido por Michael é razoavelmente preciso na obtenção das direções dos esforços principais, mesmo quando a escolha do plano de falha no mecanismo focal estiver incorreto. No entanto, a razão de forma é mais sensível à escolha adequada da falha e que a substitução do plano de falha pelo plano nodal auxiliar introduz erros significativos (Vavryčuk, 2014). Este problema foi resolvido modificando o método linear de Michael (1987b), invertendo conjuntamente para o esforço e o plano de falha. A orientação do plano de falha é determinada pela aplicação de restrição de instabilidade da falha e o esforço é calculado em interações. Como um subproduto, o atrito global sobre falhas é determinado. Testes numéricos mostram que a nova inversão interativa de esforços é rápida, precisa e funciona muito melhor que a inversão linear padrão.

Os resultados desse estudo de controle mostram que os mecanismos focais podem ser invertidos para o melhor tensor, mas a resolução é reduzida, a menos que os planos de falha possam ser escolhidos a priori. A resolução pode também ser aumentada por inclusão de restrições sobre a magnitude da tração tangencial no plano de falha.

Portanto, usar mecanismos focais para estudar pequenas variações no campo de tensão requer que outros dados (por exemplo, estudos dos hipocentros, falhamento de superfície, ou de informação estrutural, relativa à região) sejam introduzidos para escolher qual dos planos nodais é o plano de falha. No caso particular deste estudo, o plano de falha é conhecido por estudos prévios (Barros et al., 2014). 


\subsection{Referências}

Aki, K., \& Richards, P. G. (2002). Quantitative seismology. Book (p. 700). Retrieved from http://books.google.com/books?id=sRhawFG5_EcC\&printsec=frontcover|npapers2://pub lication/uuid/A2074D09-FE3A-4600-9E91-CA393C8AF127

Angelier, J. (2002). Inversion of earthquake focal mechanisms to obtain the seismotectonic stress IV-a new method free of choice among nodal planes. Geophysical Journal International, 150(3), 588-609. doi:10.1046/j.1365-246X.2002.01713.x

Barros, L. V., M. Assumpção, Chimpliganond, C. N. C., Carvalho, J. M., Huelsen, M. G. Von, Caixeta, D. F., ... Albuquerque, D. F. (2014). The Mara Rosa 2010 GT-5 earthquake and its possible relationship with the continental-scale Transbrasiliano lineament,. J. S. Am. Earth Sci. (submetido).

Benetatos, C., Málek, J., \& Verga, F. (2012). Moment tensor inversion for two microearthquakes occurring inside the Háje gas storage facilities, Czech Republic. Journal of Seismology, 17(2), 557-577. doi:10.1007/s10950-012-9337-0

Bouchon, M. (1981). A simple method to calculate Green's functions for elastic layered media. Bulletin of the Seismological Society of America, 71(4), 959-971. Retrieved from http://www.bssaonline.org/content/71/4/959.abstract

Burridge, R., \& Knopoff, L. (1964). Body force equivalents for seismic dislocations. Bull. Seismol. Soc. Am, 54, 1875-1888.

Coutant, O. (1990). Program of numerical simulation AXITRA. Laboratoire de Géophysique Interne et Tectonophysique Report, University of Joseph Fourie. Retrieved from papers://c39c63af-7d29-49f7-aaa2-85c2bd185795/Paper/p1335

Dreger, D. S., \& Helmberger, D. V. (1993). Determination of source parameters at regional distances with three-component sparse network data. Journal of Geophysical Research. doi:10.1029/93JB00023

Fan, G., \& Wallace, T. (1991). The determination of source parameters for small earthquakes from a single very broadband seismic station. Geophys. Res. Lett., 18, 1385-1388.

Fojtíková, L., Vavryčuk, V., Cipciar, A., \& Madarás, J. (2010). Focal mechanisms of microearthquakes in the Dobrá Voda seismoactive area in the Malé Karpaty Mts. (Little Carpathians), Slovakia. Tectonophysics, 492(1-4), 213-229. doi:10.1016/j.tecto.2010.06.007

Havskov, J., \& Ottemöller, L. (2010). Routine Data Processing in Earthquake Seismology - With Sample Data, Exercises and Software. Retrieved from http://www.springer.com/earth+sciences+and+geography/geology/book/978-90-4818696-9

Heidbach, O., Barth, A., Connolly, P., Fuchs, K., Müller, B., Tingay, M., ... Wenzel, F. (2004). Stress maps in a minute: The 2004 world stress map release. Eos, Transactions American Geophysical Union, 85(49), 521. doi:10.1029/2004EO490001

Jiménez, E., Cara, M., \& Rouland, D. (1989). Focal mechanism of moderate-size earthquakes from the analysis of single-station three-component surface-wave records. Bull. Seismol. Soc. Am., 79, 955-972. 
Kikuchi, B. Y. M., \& Kanamori, H. (1991). Inversion of complex body waves--iii. Physics of the Earth and Planetary Interiors, 81(6), 205-222. doi:10.1016/0031-9201(86)90048-8

Krizova, D., Zahradnik, J., \& Kiratzi, A. (2013). Resolvability of Isotropic Component in Regional Seismic Moment Tensor Inversion. Bulletin of the Seismological Society of America, 103(4), 2460-2473. doi:10.1785/0120120097

Lay, T., \& Wallace, T. C. (1995). Modern Global Seismology. International Geophysics Series (p. 521).

Maruyama, T. (1963). On the force equivalents of dynamic elastic dislocations with reference to the earthquake mechanism. Bull. Earthquake Res. Inst. Univ. Tokyo, 467-486.

Maury, J., Cornet, F. H., \& Dorbath, L. (2013). A review of methods for determining stress fields from earthquake focal mechanisms: application to the Sierentz 1980 seismic crisis (Upper Rhine graben). Bull. Soc.Geol. France, 184 (4-5), 319-334.

Michael, A. J. (1987a). Stress rotation during the Coalinga Aftershock Sequence. Journal of Geophysical Research, 92(B8), 7963. doi:10.1029/JB092iB08p07963

Michael, A. J. (1987b). Use of focal mechanisms to determine stress: A control study. Journal of Geophysical Research, 92(B1), 357. doi:10.1029/JB092iB01p00357

Nakano, H. (1923). Notes on the nature of the forces which give rise to the earthquake motions. Seism. Bull. Centr. Met. Obs. Japan, 1, 92-120.

Silver, P. G., \& Jordan, T. H. (1982). Optimal estimation of scalar seismic moment,. Geophys. J. Roy. Astron. Soc., 70, 755-787. doi:10.1111/ j.1365-246X.1982.tb05982.x

Sokos, E. N., \& Zahradnik, J. (2008). ISOLA a Fortran code and a Matlab GUI to perform multiple-point source inversion of seismic data. Computers \& Geosciences, 34(8), 967-977. doi:10.1016/j.cageo.2007.07.005

Sokos, E., \& Zahradnik, J. (2013). Evaluating Centroid-Moment-Tensor Uncertainty in the New Version of ISOLA Software. Seismological Research Letters, 84(4), 656-665. doi:10.1785/0220130002

Stein, W., \& Wysession, M. (2003). An Introduction to Seismology, Earthquakes, and Earth Structure. (M. Blackwell, Malden, Ed.) (p. 498).

Vavryčuk, V. (2001). Inversion for parameters of tensile earthquakes. Journal of Geophysical Research, 106(B8), 16339. doi:10.1029/2001JB000372

Vavryčuk, V. (2014). Iterative joint inversion for stress and fault orientations from focal mechanisms. Geophysical Journal International, 199(1), 69-77. doi:10.1093/gji/ggu224

Zahradnik, J., Serpetsidaki, A., Sokos, E., \& Tselentis, G. A. (2005a). Iterative deconvolution of regional waveforms and double-event interpretation of the 2003 Lefkada earthquake, Greece. Bull. Seismol. Soc. Am 95, 95(1), 159-172. doi:10.1785/0120040035

Zahradnik, J., \& Plesinger, A. (2005b). Long-Period Pulses in Broadband Records of Near Earthquakes. Bulletin of the Seismological Society of America, 95(5), 1928-1939. doi:10.1785/0120040210 
Zahradník, J., Janský, J., Plicka, V., Zahradnik, J., \& Jansky, J. (2008). Detailed waveform inversion for moment tensors of M 4 events: Examples from the Corinth Gulf, Greece. Bull. Seismol. Soc. Am., 98, 98(6), 2756-2771. doi:10.1785/0120080124

Zahradnik, J., \& Custodio, S. (2012). Moment Tensor Resolvability: Application to Southwest Iberia. Bulletin of the Seismological Society of America, 102(3), 1235-1254. doi:10.1785/0120110216 


\title{
CAPÍTULO 3 - ARTIGO
}

\section{Focal mechanisms and moment magnitudes of micro-earthquakes in cen- tral Brazil by waveform inversion with quality assessment and inference of the local stress field}

\author{
Juraci Carvalho ${ }^{1}$, Lucas Vieira Barros ${ }^{1}$, Jiří Zahradník ${ }^{2}$ \\ 1 - Observatório Sismológico - UnB, Brasília - DF, Brasil (juraci@unb.br, lucas@unb.br) \\ 2 - Faculty of Mathematics and Physics - Charles University in Prague, Czech Republic \\ (jiri.zahradnik@mff.cuni.cz)
}

\begin{abstract}
We investigated the ability of the full waveform inversion to retrieve focal mechanisms of 11 micro-earthquakes ( $M w 0.8$ to 1.4). The events represent aftershocks of a $5.0 \mathrm{~m}_{\mathrm{b}}$ earthquake that occurred on October 8, 2010 close to the city of Mara Rosa, state of Goiás, Brazil. The main contribution of the work lies in demonstrating feasibility of the waveform inversion of such weak events. The inversion was possible thanks to recordings available at 8 local temporary seismic stations at epicentral distances less than $8 \mathrm{~km}$, at which waveforms can be successfully modeled at relatively high frequencies $(1.5-2.0 \mathrm{~Hz})$. The obtained fault-plane solutions are in agreement with a composite focal mechanism previously calculated from first-motion polarities. They also agree with the fault geometry inferred from precise relocation of the Mara Rosa aftershock sequence. The focal mechanisms provide an estimate of the local stress field. The paper may serve as a pilot study for similar investigations in intraplate regions where the stress-field investigations are difficult due to rare earthquakes occurrence, and weak events must be studied with a detailed quality assessment.
\end{abstract}




\subsection{Introduction}

Knowledge of the crustal stress distribution is fundamental for understanding tectonics and seismicity, particularly in intraplate regions (Zoback, 1992). The vast majority of the stress-field estimates in a global scale were determined by using earthquake focal mechanisms (Heidbach et al., 2004). In Brazil, only few earthquake focal-mechanism studies have been made due to low seismicity and sparseness of the seismographic stations. Thus, more investigations of the Brazilian focal mechanisms are needed. The stress field in Brazil results from a combination of local and regional forces. Local forces are caused by structural heterogeneities in the lithosphere and thermal anomalies in the asthenosphere, e.g. ( Assumpção et al. (2004). The regional ones are related to tectonic forces originating mainly at the edges of the lithospheric plates, such as ridge-push forces in the Middle-Atlantic Ridge, and resistance forces produced in the contact of Nazca and South-American plates, causing compressive stress within the intraplate region (Assumpção, 1992).

The first studies of focal mechanisms in Brazil were carried out by Mendiguren \& Richter (1978); Assumpção et al. (1985); Assumpcao \& Suarez (1988); Assumpção (1998a, 1998b) and Ferreira et al. (1998). More recently new results were presented by Barros et al. (2009 and 2014); Chimpliganond et al. (2010) and Agurto-Detzel et al. (2014). Almost all previous fault-plane solutions were obtained by $\mathrm{P}$-wave first-motion polarities and/or the amplitude ratios of $\mathrm{P}, \mathrm{pP}$ and $\mathrm{sP}$ phases.

Various computer codes are available to calculate moment tensors at regional and local distances (e.g., TDMT_INV developed by Dreger (2003), ISOLA by Sokos \& Zahradnik (2008), FMNEAREG by Delouis et al. (2008) and Maercklin et al. (2011), KIWI by Cesca et. al. (2010) and the code of Yagi and Nishimura (2011)). Nevertheless, their application to weak events is still quite challenging because, as a rule, the micro-earthquakes $(\mathrm{Mw}<2)$ have a good signal-to-noise ratio only at relatively high frequencies (above $\sim 0.5 \mathrm{~Hz}$ ), and such waveforms can be modeled only at near seismic stations (Fojtíková et al., 2010; Fojtíková \& Zahradnik, 2014; Benetatos et al., 2012). For moment tensor inversions at very local distances and very high frequencies, typical mining applications, see, for example, Vavryčuk and Kuhn (2012) and Sen et al. 
(2013). In this paper we use ISOLA software in its latest version (Sokos \& Zahradnik, 2013); http://geo.mff.cuni.cz/ iz/isola brasilia/). For ISOLA automation, see Triantafyllis et al. (2014 - submitted).

Various methods and codes are also available for inverting stress field from focal mechanisms (e.g., Angelier, 2002; Gephart \& Forsyth, 1984 and Michael, 1987). As shown by Vavryčuk (2014), Michael's method is reasonably accurate when retrieving the principal stress directions even when the selection of fault planes is incorrect. However, the shape factor is more sensitive to the correct plane identification. Therefore, we use the STRESSINVERSE code developed by Vavryčuk (2014) in which Michael's method is modified by inverting jointly for the stress and true fault orientation.

On October 8, 2010, an earthquake of magnitude $5.0 \mathrm{mb}_{\mathrm{b}}$ and intensity $\mathrm{VI}$ (MM) startled residents in the northern region of the Goiás state, Brazil, having also been felt in Brasília and Goiania cities, situated as far as $300 \mathrm{~km}$ away (Barros et al., 2014). The epicenter was located with a $\sim 5 \mathrm{~km}$ accuracy, thus qualifying this event to be one of the first few GT5 (Ground Truth) earthquakes in Brazil (Barros et al., 2014). Due to proximity of the epicenter to the city of Mara Rosa, hereafter we call it Mara Rosa (or simply MR) earthquake. This event is the largest ever recorded in the Goiás Tocantins Seismic Zone (GTSZ), so far characterized by low magnitude events (Figure 3.1). Due to the low magnitudes and sparseness of seismographic stations in GTSZ, only one focal mechanism had been reported before the MR earthquake, i.e. the Brasilia event $\left(3.7 \mathrm{mb}_{\mathrm{b}}\right)$ of November 20, 2000 (Assumpção et al., 2014).

The MR event was followed by an aftershock sequence recorded by a temporary seismic network. Therefore, the aim of this paper is to investigate focal mechanisms and moment magnitudes of these weak events, mainly by the waveform inversion, and to make inferences about the local stress field. In contrast to usual standard procedures this paper aims to justify the focal mechanism results as much as possible using various quality assessments applied both to input data and output results. 


\subsection{Geological and geophysical setting}

The study area (Figure 3.1), is located in the Goiás Tocantins Seismic Zone (GTSZ) is located. Seismicity of this zone is characterized by lowmagnitude events with distribution parallel, but not coincident, with the largescale Transbrasilian Lineament (TBL).

The SW-NE oriented TBL crosses the study area from the north of the Parana basin and ending to the south of Parnaiba basin. It is characterized by high gravity anomalies along the Tocantins Araguaia belt (Assumpção et al., 1986; Assumpção \& Sacek, 2013; Fernandes et al., 1991). The area is characterized by folds and thrusts, denominated Brasilia belt that resulted from the collision and convergence of three continental plates: the Amazon craton (West), São Francisco craton (East) and Paranapanema craton (SouthWest), presently covered by the Parana basin (Figure 3.1 inset). The TransBrasilian Lineament is composed by a set of geological features formed in the Neoproterozoic, located in the convergence of the São Francisco craton and the Amazonian craton during the formation of the eastern part of the super continent Gondwana (Fuck et al., 1994; Fuck, 1994 and Cordani et al., 2013). One of the most important questions is whether the Mara Rosa sequence activated some of the known old faults of the region, and what is the relation of these faults to the local stress field derived from the seismic data. 


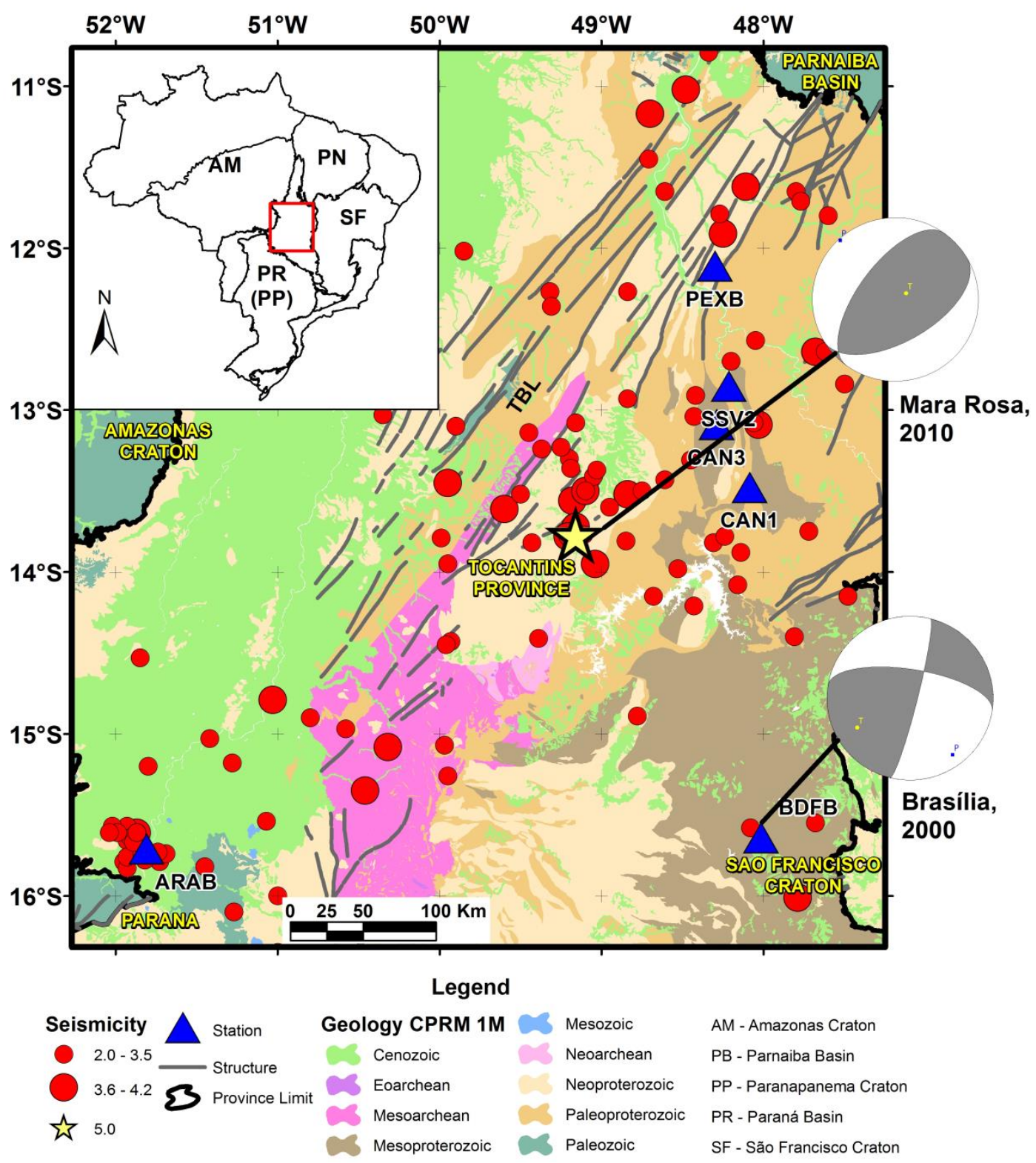

Figure 3.1 - Seismicity (red circles) of Goiás-Tocantins Seismic Zone up to 2014 (Brazilian Seismic Bulletin - SISBRA), geological map with structures of the Transbrasiliano Lineament (CPRM, 2004) and regional seismic stations (blue triangles).The focal mechanisms of the 2000 and 2010 events are taken from Assumpção et al. (2014) and from Barros et al. (2014), respectively.

\subsection{Local network}

Seismic network - A local temporary network comprising eight stations (Figure 3.2) was deployed a few days after the Mara Rosa $5.0 \mathrm{~m}_{\mathrm{b}}$ earthquake. The network was implemented with broadband (30 sec to $100 \mathrm{~Hz}$ ) and short period seismometers ( $1 \mathrm{sec}$ to $100 \mathrm{~Hz}$ ), both coupled to a 24-bit digitizer. All the data was recorded with $200 \mathrm{~Hz}$ sampling. 


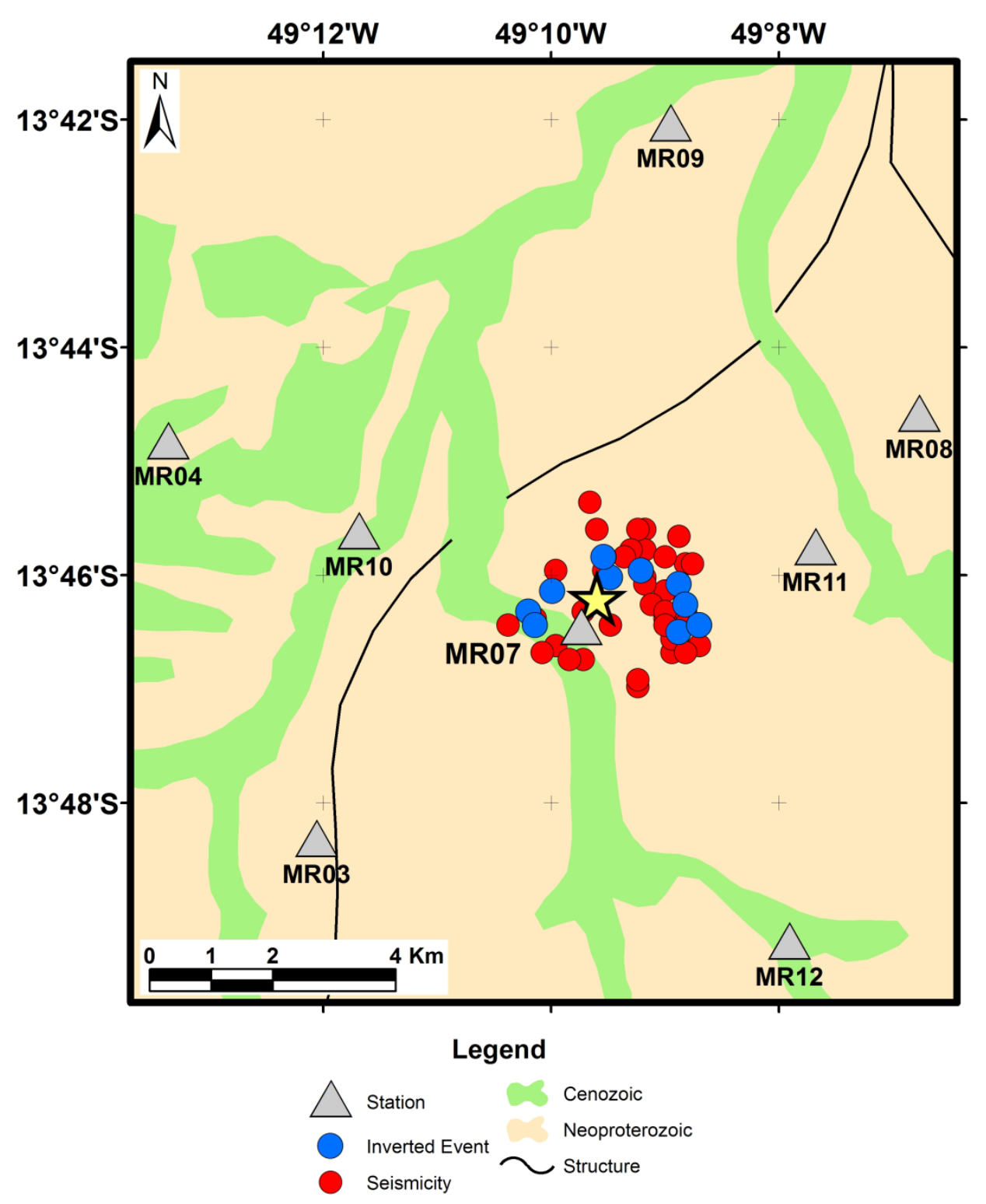

Figure 3.2 - Mara Rosa local seismographic network (triangles) and local seismicity from Oct/2010 to Jun/2011 (magnitudes <2.0). Red circles are the 53 best located events with Hypocenter and relocated with HypoDD. The 11 events shown in blue are inverted for their focal mechanism in this paper (Two events overlapped to the same location). The star denotes the mainshock $5.0 m_{b}$ of October 8, 2010.

Event location - The seismic sequence following the MR mainshock was monitored by the local network for eight months, from October 2010 to June 2011. In this period, more than 600 events were detected. The HYPOCENTER code (Lienert, 1994), implemented in SEISAN Earthquake Data Analysis software (Havskov \& Ottemöller, 2008), was used to locate the sequence. We adopted the crustal structure and P velocities from Soares et al. (2006) and the 
$\mathrm{Vp} / \mathrm{Vs}$ (1.70) from receiver function studies (França, 2004; França \& Assumpção, 2004).The density was calculated from Vp using empirical formula, and quality factors $Q p, Q s$, were only roughly estimated. The model is shown in Table 3.1 and Figure 3.3.

Table 3.1 - Velocity model $(\mathrm{Vp} / \mathrm{Vs}=1.70)$

\begin{tabular}{ccccc}
\hline $\begin{array}{c}\text { depth } \\
(\mathrm{km})\end{array}$ & $\begin{array}{c}\mathrm{Vp} \\
(\mathrm{km} / \mathrm{s})\end{array}$ & $\begin{array}{c}\text { Rho } \\
\left(\mathrm{g} / \mathrm{cm}^{* *} 3\right)\end{array}$ & $\mathrm{Qp}$ & Qs \\
\hline 0.0 & 6.0 & 2.900 & 100 & 50 \\
\hline 12.0 & 6.6 & 3.020 & 100 & 50 \\
\hline 25.0 & 6.8 & 3.060 & 100 & 50 \\
\hline 35.0 & 7.2 & 3.140 & 300 & 150 \\
\hline 38.0 & 8.3 & 3.360 & 300 & 150 \\
\hline
\end{tabular}

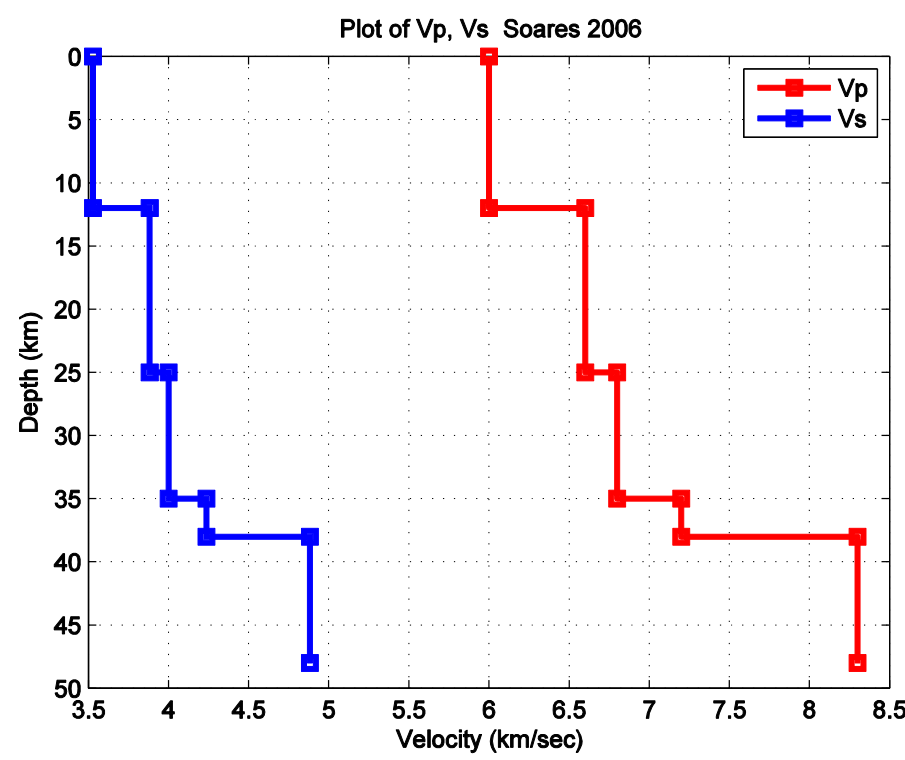

Figure 3.3 - Velocity model adopted in this paper for waveform inversion. (Derived from Soares et al., 2006).

From the 600 located events, 53 were determined with high accuracy (6 to 8 stations, impulsive $\mathrm{P}$ and $\mathrm{S}$ readings, formal horizontal error better than 0.5 $\mathrm{km})$. Finally, these events were relocated by the HypoDD relative technique (Waldhauser, 2001), see Figure 3.2.

Selecting events, stations and data quality control - An additional selection was made to find suitable events and stations for the waveform inversion. The selection criteria were adopted as follows: events within the group of 53 processed with HypoDD, magnitudes ranging from 1.2 to $2.0 \mathrm{M}_{\mathrm{D}}$, recorded by 
more than 5 local stations $(<8 \mathrm{~km})$ and being free from instrumental, cultural or electronic noise at a minimum of three stations.

To prevent biasing the focal-mechanism calculations due to problematic data, a thorough visual inspection of raw waveforms was made. We concentrated on several issues, such as, possible data gaps, clipping, electronic noise, excessive microseismic and/or cultural noise, low signal-tonoise ratio (SNR). When detected, the records were removed from processing. Examples of these problems are shown in Figures 3.4a and 3.4b. Figure 3.4a shows the waveforms of the station MR9, event 10. The signal is from a broadband instrument output, band-pass filtered (1.5 to $2.0 \mathrm{~Hz})$ and is contaminated by an excessive cultural noise. Figure $3.4 \mathrm{~b}$ shows the waveforms of the station MR3, event Ev6. The signal is from a broadband instrument output, band-pass filtered (1.5 to $2.0 \mathrm{~Hz}$ ) and is contaminated with an electronic monochromatic noise, probably, from equipment malfunction.

A particular attention was devoted to the instrumental disturbances described by Zahradnik \& Plesinger $(2005,2010)$ and Vackáŕ et al. (2015). They can be easily detected by inspecting the output (raw velocity) without any preliminary band-pass filtering and prior removal of the instrument response, or, if the disturbances are weaker, by inspecting the integrated output (raw displacement). If overlooked, the disturbances can harm the moment-tensor inversion. Such disturbances are demonstrated in Figures 3.4c and 3.4d. Figure 3.4c shows the waveforms from the station MR8, event Ev6. The signal is from a broadband sensor (raw velocity) instrument output, unfiltered and uncorrected. Figure $3.4 \mathrm{~d}$ is similar, showing the station MR11 (short period sensor), event Ev6. All (3) seismograms with detected disturbances like this were removed from processing. Finally we arrived at 11 selected events shown in Figure 3.2 and detailed in Table 3.2. Note that all the events are very shallow $(<2 \mathrm{~km})$. The cause and nature of this disturbances are well explained in Zahradnik \& Plesinger (2005, 2010). 


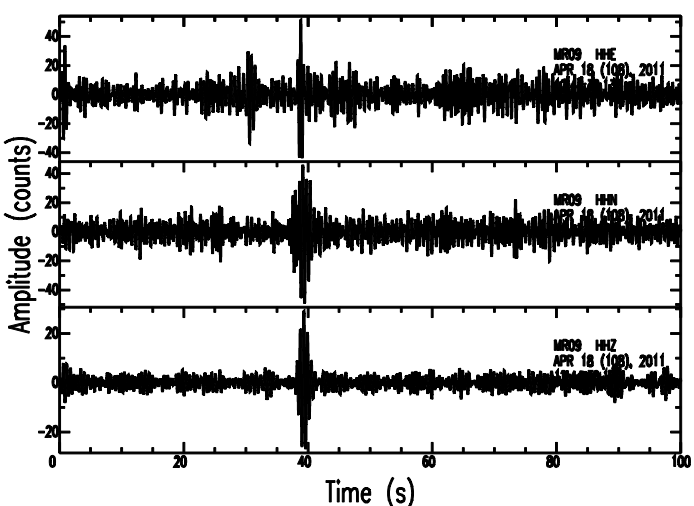

4a)

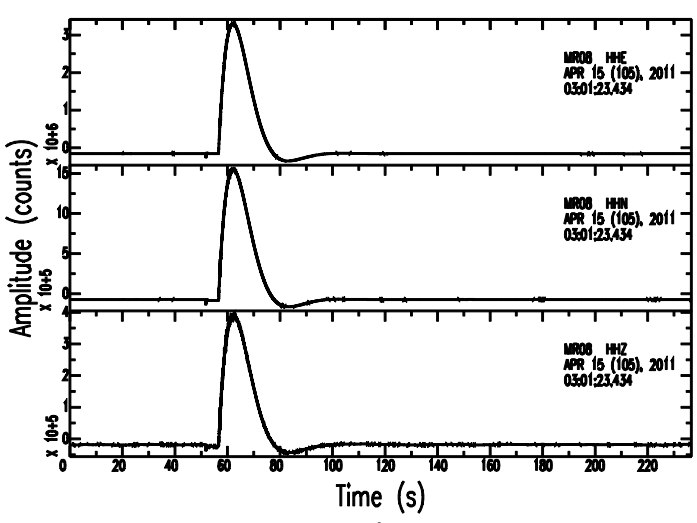

4c)

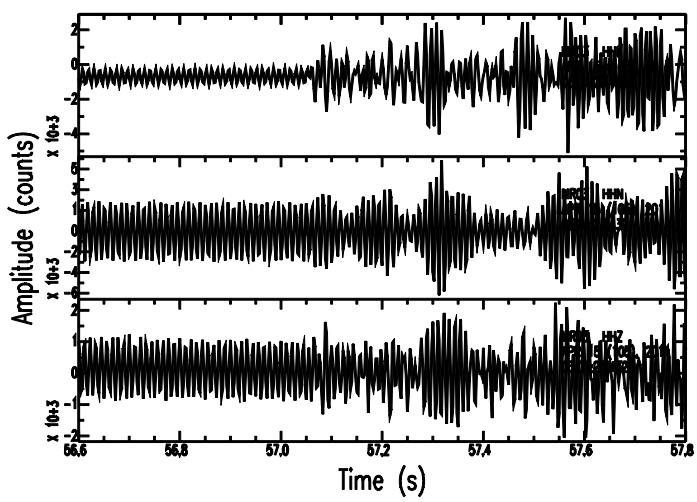

$4 b)$

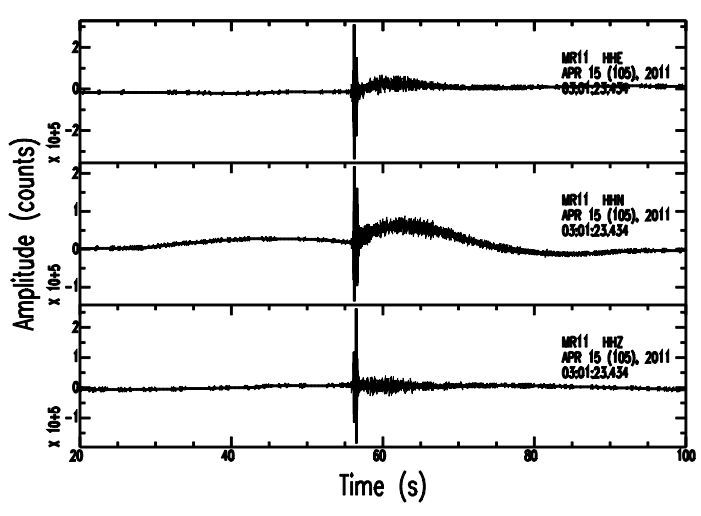

$4 d)$

Figure 3.4 - Waveform quality check showing data problems, including: a) excessive cultural noise present at all components (Ev10, MR09, filtered 1.5-2.0 Hz); b) monochromatic noise present at all components (EV6, MR3, filtered 1.5-2.0 Hz); c) instrumental disturbance present at all components (Ev6, MR08, raw data); and d) instrumental disturbance present at the horizontal components (Ev6, MR11, raw data).

Table 3.2 - Events selected for the waveform inversion.

\begin{tabular}{|c|c|c|c|c|c|c|c|}
\hline \multirow{2}{*}{ Event Number } & \multirow{2}{*}{$\begin{array}{c}\text { Date } \\
\text { (yyyy/mm/dd) }\end{array}$} & \multirow{2}{*}{$\begin{array}{l}\text { Origin Time } \\
\text { (UTC) }\end{array}$} & \multicolumn{2}{|c|}{ Coordinates } & \multirow{2}{*}{$\begin{array}{c}\text { Depth } \\
\text { (HypoDD) } \\
(\mathrm{km})\end{array}$} & \multirow{2}{*}{$\begin{array}{l}\text { Magnitude } \\
\qquad\left(\mathrm{M}_{\mathrm{D}}\right)\end{array}$} & \multirow{2}{*}{$\begin{array}{l}\text { RMS } \\
\text { (sec) }\end{array}$} \\
\hline & & & Lat. $\left({ }^{\circ}\right)$ & Long. $\left({ }^{\circ}\right)$ & & & \\
\hline 1 & $2011 / 03 / 30$ & $21: 40: 15.3$ & -13.7757 & -49.1480 & 0.8 & 1.4 & 0.03 \\
\hline 2 & $2011 / 04 / 03$ & 09:19:49.49 & -13.7685 & -49.1478 & 1.2 & 1.3 & 0.03 \\
\hline 3 & $2011 / 04 / 03$ & 15:51:08.60 & -13.7667 & -49.1585 & 1.4 & 1.3 & 0.04 \\
\hline 4 & $2011 / 04 / 04$ & 10:19:46.61 & -13.7645 & -49.1590 & 1.3 & 1.2 & 0.04 \\
\hline 5 & $2011 / 04 / 09$ & 19:52:13.16 & -13.7660 & -49.1535 & 1.7 & 1.5 & 0.03 \\
\hline 6 & $2011 / 04 / 15$ & 03:02:19.55 & -13.7743 & -49.1440 & 0.6 & 1.3 & 0.03 \\
\hline 7 & $2011 / 04 / 15$ & 03:05:35.15 & -13.7743 & -49.1440 & 1.4 & 1.2 & 0.03 \\
\hline 8 & $2011 / 04 / 16$ & 09:01:07.67 & -13.7715 & -49.1462 & 1.0 & 1.2 & 0.03 \\
\hline 9 & $2011 / 04 / 18$ & $06: 42: 20.83$ & -13.7723 & -49.1695 & 1.3 & 1.4 & 0.02 \\
\hline 10 & $2011 / 04 / 18$ & $12: 45: 13.62$ & -13.7742 & -49.1690 & 1.2 & 2.0 & 0.02 \\
\hline 11 & $2011 / 04 / 28$ & $13: 16: 36.23$ & -13.7690 & -49.1665 & 0.9 & 1.4 & 0.02 \\
\hline
\end{tabular}




\subsection{Waveform inversion}

Method - The waveform inversion developed by Sokos \& Zahradnik (2008 and 2013), implemented in the ISOLA software, is based on the least-squares calculation of the moment tensor and a grid search of the centroid position and time. A single point-source approximation is used, and the moment-rate time dependence is assumed to be known (in this paper we use the delta function). Complete displacement waveforms are used (without pre-selecting any phases); the inversion is made in the time domain. The Earth response is represented by Green's functions calculated in a 1D velocity model, using the discrete-wavenumber method of (Bouchon, 2003; Gazzani, Daglia, \& Papetti, 2012) and Coutant (1990). This method includes the near-, intermediate-, and far-field terms. The grid search of centroid in this work is done with the epicenter position fixed and varying the depth from $0.4 \mathrm{~km}$ up to $2 \mathrm{~km}$, in steps of $0.2 \mathrm{~km}$. The temporal grid search is made +/- 1 second around the locationprovided origin time, in steps of $0.09 \mathrm{sec}$. These parameters were set up after preliminary tests aimed at using the same processing scheme for all events.

The frequency band suitable for the waveform inversion is constrained by two main factors. The high-frequency limit is given by the accuracy of the velocity model; due to approximate nature of the velocity models, only relatively low frequencies can be successfully modeled. In other words, the modeling is feasible up to epicentral distances equivalent to a few minimum shear wavelengths only (Fojtikova and Zahradnik, 2014). When decreasing the source-station distance, the results are less affected by the limited accuracy of the crustal model hence the high-frequency limit increases. Moreover, if (for simplicity) we assume that the moment rate is a delta function, the high-frequency limit is also determined by the corner frequency of the earthquake, i.e.: the maximum inverted frequency must be lower than the event corner frequency. The lowfrequency inversion limit is given by the availability of a good signal above noise. As a rule, weak events have a poor SNR below the microseismic noise peak ( $0.2 \mathrm{~Hz})$. A special tool exists in ISOLA to calculate SNR in the spectral domain. For maximum homogeneity of the processing, we selected events and stations allowing applicability of a constant frequency range of $1.5-2.0 \mathrm{~Hz}$. 
In this paper we assume a deviatoric moment tensor. It is represented by means of the scalar moment Mo or the moment magnitude $\mathbf{M w}$, by the strike/dip/rake (hereafter denoted $\mathrm{S} / \mathrm{D} / \mathrm{R}$ ) angles and the azimuth and plunge angles describing the $\mathrm{P}$ and $\mathrm{T}$ axes. The shear part of the moment tensor is quantified by the double-couple percentage (DC\%), using Eq. 8 of (Vavryčuk, 2001). The waveform fit between the observed and synthetic displacement records at all used stations is measured by correlation (corr) and global variance reduction (VR); see Eqs. 9, 11 and 13 of Křižová et al. (2013). An example of the waveform fit is presented in Figure 3.5, where the variance reduction of the individual components is also shown for the event 9. Variance reductions for all eleven events are shown in the Annex II figures from II.1 to II.11.
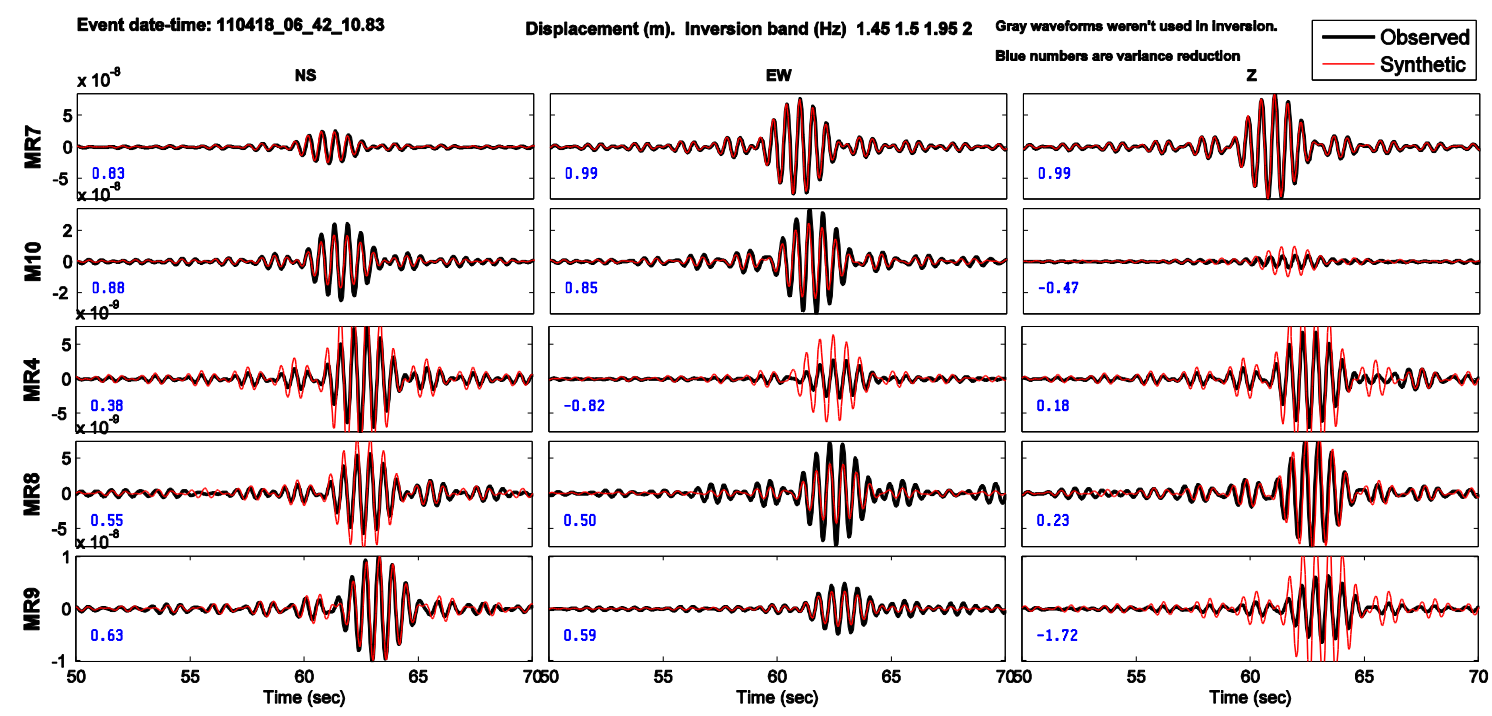

Figure 3.5 - Comparison between the observed (black) and synthetic (red) waveforms. The example refers to Event 9 of Tables 3.2 and 3.3. The waveform match is quantified by the variance reduction values per station components (blue numbers). The horizontal time axis is in seconds, $t=50 \mathrm{sec}$ corresponds to 10 seconds after the origin time. The vertical axis indicates the displacement amplitude in meters.

While the above listed issues became already more or less standard, at least for larger magnitudes, little attention has been, so far, devoted in various network centers and agency routine reports to possible errors of the focal mechanisms in terms of their strike, dip and rake angles. Naturally, a good quality of the waveform fit (high VR) represents a necessary condition to qualify an event as successfully resolved, but it is not sufficient. If the source-station distri- 
bution is not favorable, the inverse problem may be ill-posed. This can be signalized by high values of the condition number (CN), Eq. 18 of Křížová et al. (2013).

A more detailed insight into the uncertainty of the $S / D / R$ angles may be obtained by the resolvability analysis proposed by Zahradník \& Custódio (2012), and we shall use the latter method to demonstrate the uncertainty of the focal mechanisms by means of the so-called scatter plots of nodal lines. Using ISOLA, the scatter nodal-line plot (e.g. Fig. 6 of Sokos \& Zahradnik (2013) is calculated as a discrete sample of the moment-tensor solutions inside the theoretical six-dimensional error ellipsoid. The uncertainty of each event is then quantified by themean value of the Kagan angle (Kagan, 1991) measured between the $\mathrm{S} / \mathrm{D} / \mathrm{R}$ best solution of the event under consideration and each solution of the scatter plot. For brevity, this mean difference is called 'confidence interval' in the present paper. It serves as a relative comparison of the uncertainty among the studied events. It has no strict statistical meaning of the confidence limits (chapter 15 of Press et al. (1997), because it is difficult to estimate the input waveforms data error (Michele et al., 2014).

Results - Summary of the results for all 11 studied events is in Table 3. Each event is described by the parameters defined in the preceding sections, i.e., SNR, DC\%, CN, VR, $\mathbf{M}_{\mathbf{D}}, \mathbf{M w}$, both, the location and centroid depth, S/D/R angles and the P- \& T-axes angles. The scatter nodal-line plot and the confidence interval for each event are presented in the Figure 3.6.

\begin{tabular}{|c|c|c|c|c|c|c|}
\hline Event & EV1 & EV2 & EV3 & EV4 & EV5 & EV6 \\
\hline $\begin{array}{c}\text { Confidence } \\
\text { interval ( }\end{array}$ & 9.1 & 7.8 & 20.6 & 10.4 & 10.0 & 10.1 \\
\hline $\begin{array}{c}\text { Nodal line } \\
\text { scatter } \\
\text { beachball }\end{array}$ & 0 & 0 & 0 & 0 & 0 & \\
\hline
\end{tabular}

\begin{tabular}{c|c|c|c|c|c} 
Event & EV7 & EV8 & EV9 & EV10 & EV11 \\
\hline $\begin{array}{c}\text { Confidence } \\
\text { interval ( }\end{array}$ & 11.2 & 10.0 & 14.4 & 13.0 & 14.9 \\
\hline $\begin{array}{c}\text { Nodal line } \\
\text { scatter } \\
\text { beachball }\end{array}$ & 0 & 0 & 0 & 0 & \\
\hline
\end{tabular}

Figure 3.6 - Uncertainty of the focal mechanisms calculated in the present paper, characterized by the scatter plots of nodal lines and the corresponding confidence intervals. 
Inversion assessment - Basically, we require inversion results with high values of VR and low values of $\mathbf{C N}$. Since the studied events are of tectonic origin, we also assume that the inversions providing high DC\% are preferable. Also preferable are the inversions of high SNR data. As explained in Sokos \& Zahradnik (2013), the output check is difficult because the quality measures like VR, CN, DC\% and confidence intervals are only relative. Therefore, we first investigate all 11 events, set up the range of the individual quality parameters and then try to understand which of the results are better or worse in a relative sense.

The SNR values varied from 1 to 7 , that is why we consider preferable the results obtained for $\mathbf{S N R}>=2$, while those of $\mathbf{S N R}=1$ are classified as 'poor' (Events 2 and 8, marked by blue color in column 3 of Table 3.3). The DC\% is high for all events, ranging between $71 \%$ and $99 \%$, thus not indicating any event as poor. The same applies for $\mathbf{C N}$, ranging from 3.8 to 6.4 , with exception of Event $3(\mathbf{C N}=10.8)$; the $\mathbf{C N}$ interval $3.8-6.4$ is narrow because the sourcestation configurations are similar and the frequency range is identical for all events. Also the confidence interval (20.6) of Event 3 is greater than the other events-(Figure 3.6). Note that Events 2 and 5 have been also processed with as few as three stations only, but their $\mathbf{C N}$ is better (lower) than Event 3 due to different source-station configurations. It is a good illustration of the fact that just the number of stations in the waveform inversion is not a good indicator of the reliability of the results.

The variance reduction is an important quality indicator, although not simple, because the individual events were processed with unequal number of records. The variation of VR from 0.27 to 0.94 is large, and we can identify three worst cases (Events 1, 7 and 8); they are characterized by $V R<0.4$. Note also that the above discussed Event 3, with its poor (large) $\mathbf{C N}$ value, has a relatively high VR. It is a good illustration of the limited meaning of the high VR values in case of an ill-posed problem, in which data are fitted very well, but the solution has a very limited reliability. Although well known in theory, this feature of the moment-tensor inversion is often overlooked in practice when the number of stations is small. 
The difference between $\mathbf{M}_{\mathbf{D}}$ and $\mathbf{M w}$ is between 0 and 1.10 (in the absolute value sense) and the three worst cases (Events 1, 3 and 10) have $\mid \mathbf{M}_{\mathbf{D}^{-}}$ $\mathbf{M w} \mid>=0.5$. The (absolute-valued) difference between the location depth and the centroid depth is between 0 and $0.9 \mathrm{~km}$. The two worst cases (Events 3 and 7) have the difference $>0.5 \mathrm{~km}$.

Another important quality measure of the inversion output is how the moment-tensor solutions agree with first-motion polarities. The relatively highfrequency and narrow band inversion, like in the present paper, might introduce ambiguity to the $\mathrm{P} \& \mathrm{~T}$ axes, accompanied by a half-period error in the centroid time. See, for example, p. 163 and Figure 3.3 of Zahradník et al. (2005), or p. 2760 of Zahradník at al. (2008). If all (or most) observed polarities disagree with the inverted focal mechanism, the correct mechanism could be found easily, simply by changing the computed value of the rake into rake minus $180^{\circ}$. We have not found any case like this in our analysis. If however some polarities are satisfied and some are not, the focal-mechanism solution is less reliable, and acceptance of the solution depends on subjective decision of how many polarity misfits are acceptable. We made careful polarity readings from all three components and checked their consistency with the source-station configuration. Unclear polarities, or those projected close to nodal lines, were discarded. Thus, four and six events had zero and one misfit, respectively; these are classified as the polarity agreement in column 9 of Table 3.3. The worst case of Event 6 (two misfits) is classified as not passing the polarity criterion.

Using all the above criteria, the individual problematic parameters are marked in Table 3.3 by blue color. Most of the 11 studied events have at least one problematic quality indicator, thus we conclude that all focal mechanisms are of a comparable quality. In other words, and having in mind that the classification is partly subjective, we do not have any strong reason for discarding any of the events (Figure 3.2). 
Capítulo 3 - Artigo

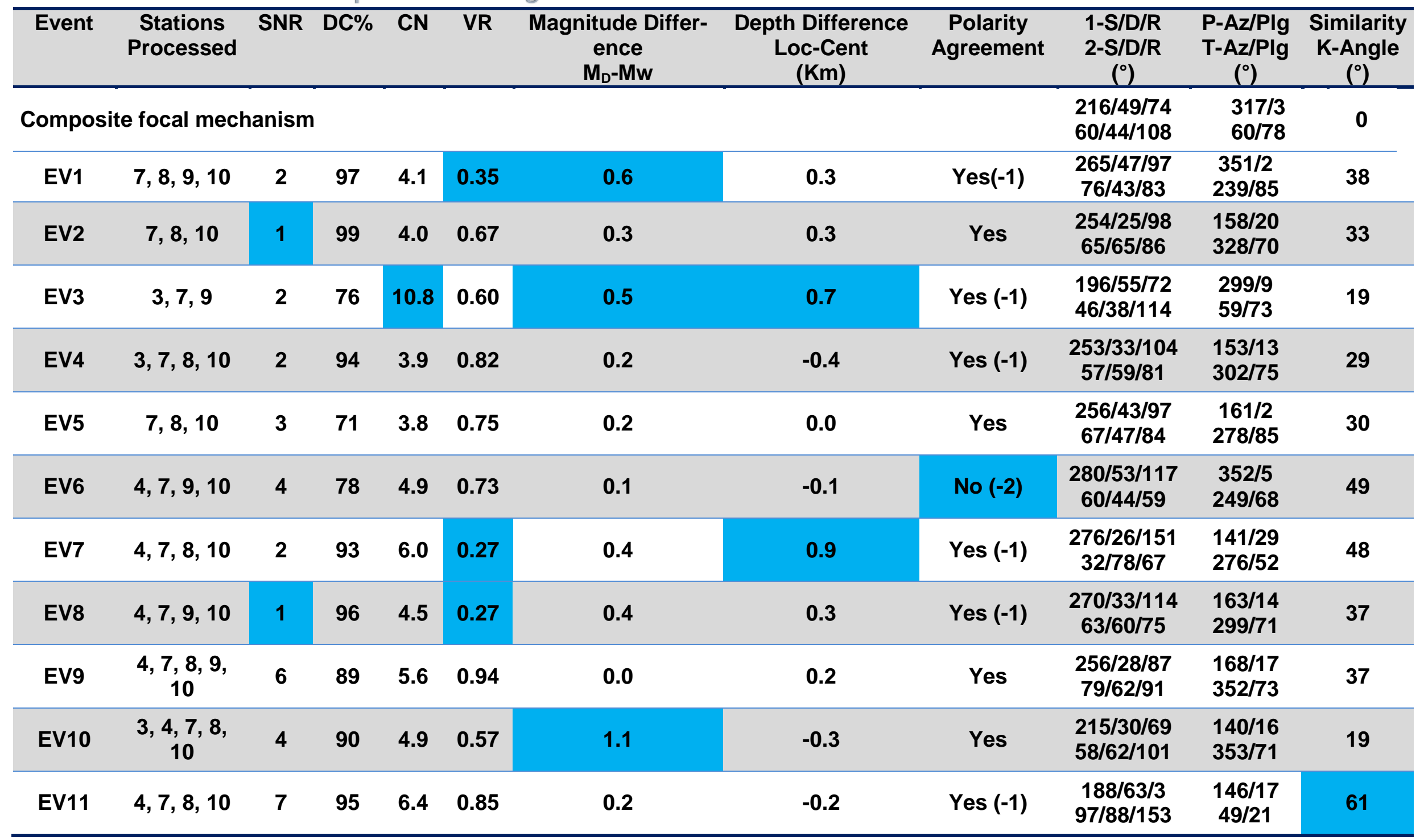

Table 3.3-Summary of the results. See also Figure 3.6. All events were inverted in the frequency range 1.5-2.0 Hz. The boxes marked with blue color indicate problematic cases. For each event we calculated the angular deviation with respect to the composite focal mechanism (in terms of the Kagan angle), and present it in the last column as Similarity. 
Similarity of events - The composite focal mechanism of the Mara Rosa sequence (Barros et al., 2014) is shown in the first row of Table 3.3 and in Figure 3.7a. To measure similarity of the mechanisms, we take the composite solution as a reference. For each event we calculate its angular deviation with respect to this reference mechanism (in terms of the Kagan angle), and present it in the last column of Table 3.3. For simplicity of Table 3.3, we call this angular deviation 'Similarity'. It varies between $19^{\circ}$ and $49^{\circ}$, with one exception - Event 11 - characterized by the $61^{\circ}$ deviation from the reference. This comparison has two implications: (i) Events 1 to 10 have a good degree of mutual similarity, but, as a whole, they show a certain rotation from the composite solution. (ii) Event 11 is clearly different. The anomaly of Event 11 relates mainly to its $T$ axis. All the obtained focal mechanisms, except Event 11, are summarized in Figure 3.7b. We have no explanation for the anomaly of Event 11 , because its quality control measures do not deviate significantly from the other events.

7a)

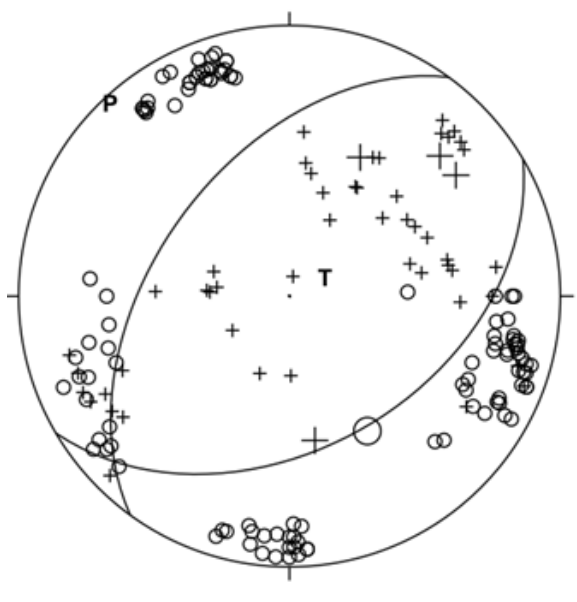

7b)

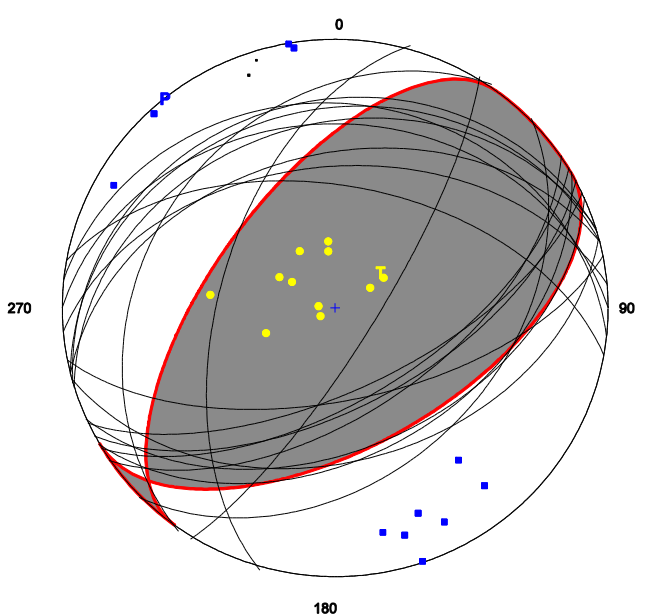

180

Figure 3.7 - Comparison of the focal mechanism solutions: (a) the composite first-motion polarity solution; small symbols are clear polarity data from aftershocks recorded by the local network; large symbols are clear polarity data of the mainshock at regional stations. (b) the waveform inversion of the present paper (Events 1 to 10 of Table 3.3). The blue and yellow dots represent the $P \& T$ axes respectively, the red line and shading in panel $(b)$ is the same solution as in panel (a).

The events of Figure 3.7b (plus the non-similar Event 11) are also displayed in Figure 3.8, where the solutions are shown in terms of their P-axes projected onto the horizontal plane. The results are roughly consistent with the regional stress field (NW-SE maximum compression) in the large zone of 
central Brazil, indicated by the blue bar in the Figure 3.8, according to theoretical studies (Coblentz \& Richardson, 1996) and in situ measurements performed by Caproni \& Armelin (1990). The anomaly of Event 11 is again obvious when comparing the 'beachballs', but its P-axis orientation is close to the other events.

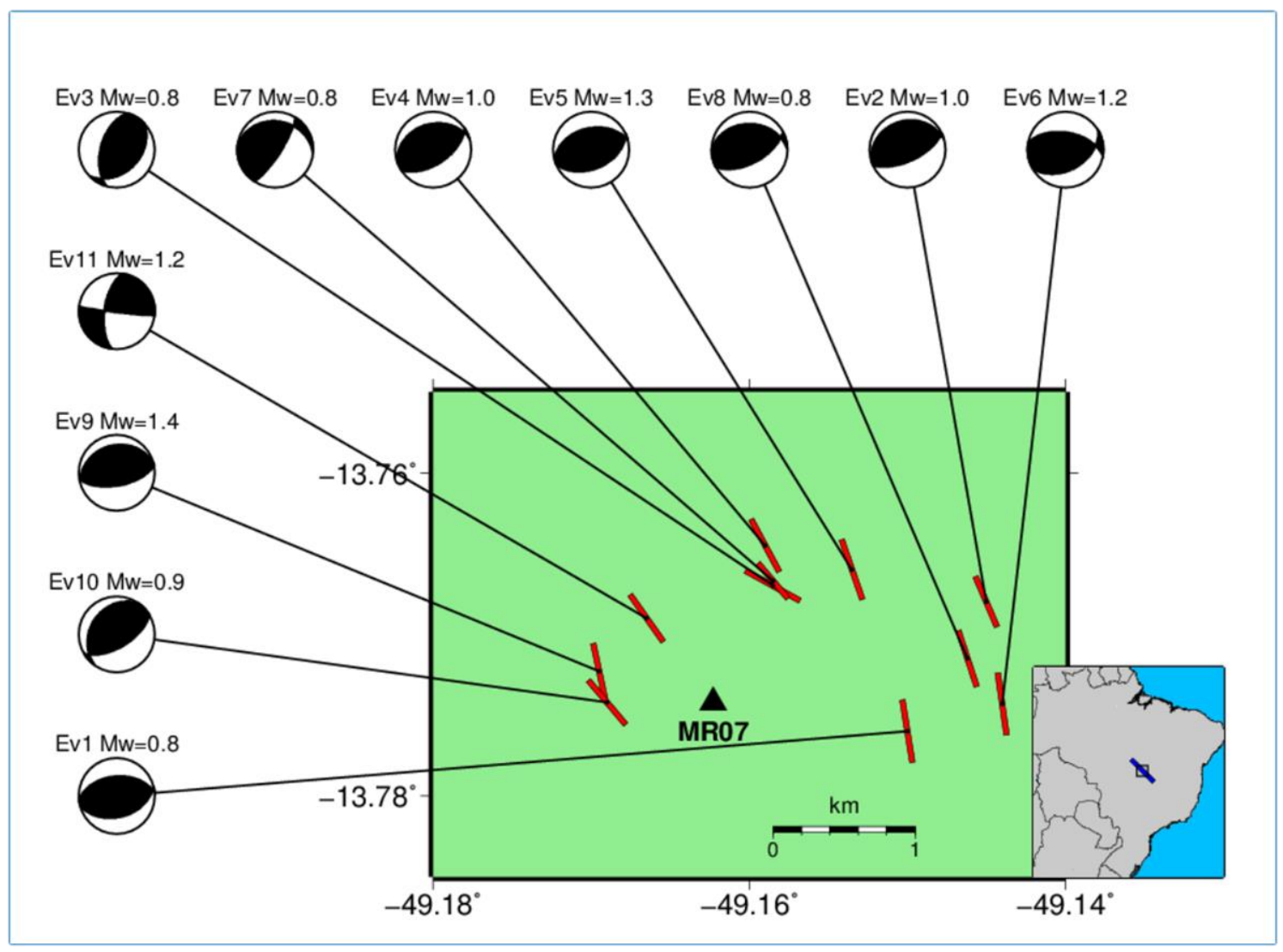

Figure 3.8 - Focal mechanisms derived in this paper and the corresponding horizontal projections of their $P$ axes (red bars) for the investigated events of Table 3. Blue bar in the inset indicates the NW-SE regional compression according to Caproni \& Armelin (1990).

Independent validation - Since the focal mechanisms have revealed their mutual similarity, all aftershocks might belong to the same fault system and the faults were mobilized by stresses with similar orientation. It is therefore logical to ask which of their nodal planes could be identified with the fault planes. Excluding Event 11, the 'first' nodal planes (i.e. those in the top rows of columns 10 and 11 in Table 3.3) have their strike $196^{\circ}$ to $280^{\circ}$ and dip $25^{\circ}$ to $55^{\circ}$. These values can be compared with the spatial distribution of the 53 relocated aftershocks (previously shown in Figure 3.2). Figure 3.9 shows that the aftershocks clustered along the best-fitting plane characterized by strike $244^{\circ}$ 
and dip $26^{\circ}$ (Barros et al., 2014). That is why we believe that most of the studied events might have ruptured along their first nodal plane, i.e. along the fault planes striking at $196^{\circ}$ to $280^{\circ}$.

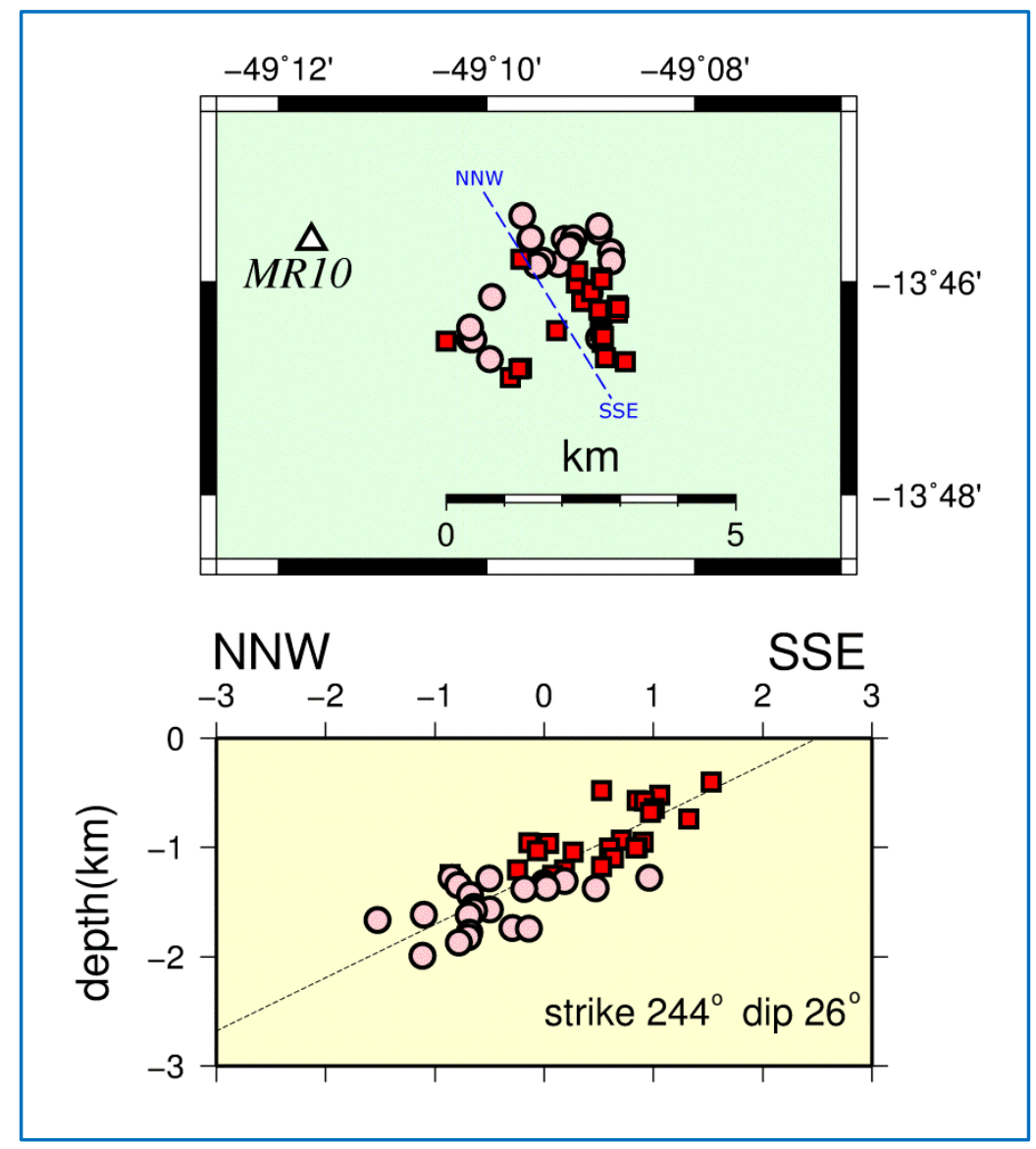

Figure 3.9 - The HypoDD relocated aftershocks in map view (top) and in a vertical crosssection (bottom), compared to a plane best fitting their distribution (strike $244^{\circ}$, dip $26^{\circ}$ ) after Barros et al. (2014). The cross-section is plotted (NNW-SSE) perpendicularly to the $244^{\circ}$ strike. The red squares and pink circles indicate the shallower and deeper events, respectively, choosing the limit between them tentatively at a depth of $1.3 \mathrm{~km}$. The station MR10 (triangle) is shown as a reference.

Stress field - As already mentioned above in connection with Figure 3.8, the P axes of the studied events are in a rough agreement with the expected regional stress field. The calculated focal mechanisms allow us to obtain some quantification of the local stress field. To this goal we use the STRESSINVERSE code of Vavryčuk (2014). In principle, the code calculates not only directions of stress axes and the relative size of the principal stresses (the shape factor), but it enables also determining fault friction and resolving ambiguity of nodal planes for each studied event. Moreover, two optimally oriented planes and slip directions on them can be determined, too. These are 
collectively referred to as 'principal earthquakes' in the code, or simply 'optimally oriented faults' in this paper.

In practice, when the input data (i.e., the strike, dip and rake angles of the studied focal mechanisms) are limited, the stress inversion method can resolve only few of the stress parameters. Various preliminary tests have been carried out to understand which of the 11 studied events can/should be included in the inversion, and which stress parameters can be resolved. We have found that a stable result (constant with respect to varying data subsets) is the orientation of the stress axes when the anomalous Event 11 is excluded. The data are not sensitive to friction hence we fix it at the 0.6 value. The best-fitting $\sigma_{1}, \sigma_{2}$ and $\sigma_{3}$, axes (the maximum, intermediate and minimum compression) are then those shown in Figure 3.10a and Table 3.4. The $\sigma_{1}$ and $\sigma_{2}$ axes are nearly horizontal; the $\sigma_{3}$ axis is nearly vertical, corresponding to the reverse faulting regime. The two optimally oriented faults are also given in Table 3.4. Their angular deviation with respect to the $\sigma 1$ axis is $30^{\circ}$. An acceptable quality of the stress inversion is confirmed by Figure $3.10 \mathrm{~b}$, because the studied events are relatively well clustered along the outer Mohr's circle. All 10 events are situated in the lower semi-circle, implying that only one of the two optimally oriented faults of the region was activated, i.e. the reverse fault striking at $264^{\circ}$, weakly dipping at $16^{\circ}$, with rake $111^{\circ}$. (These parameters are relatively stable with respect to the poorly resolved friction; for example, when fixing the friction at the 0.2 value, we obtain the optimally oriented fault with strike $245^{\circ}$, dip $25^{\circ}$ and rake $100^{\circ}$.) The inversion with friction 0.6 was repeated 10 times, each time removing one input mechanism. Two cases were ill-posed, the others provided the following variation of the optimally oriented faults: strike $255^{\circ}-269^{\circ}$, dip $15^{\circ}$ $16^{\circ}$, rake $103^{\circ}-116^{\circ}$. The uncertainty estimate (a relative confidence) of the stress axes provided by the STRESSINVERSE code is shown in Figure 3.10c. It represents the variation of the stress axes when the input mechanisms are varied 500 -times, assuming $25^{\circ}$ uncertainty of each. The three axes have comparable uncertainties. The shape ratio has not been well resolved hence it is not discussed here. The results obtained by joint inversion of the stress and fault planes have been very close to those obtained under the assumption that we identify all fault planes with the nodal planes striking at $196^{\circ}$ to $280^{\circ}$. 
Table 3.4 - Principal stress axes and the optimally oriented faults derived from Events 1 to 10.

\begin{tabular}{|c|c|c|c|c|c|}
\hline $\begin{array}{l}\text { Stress } \\
\text { axis }\end{array}$ & $\begin{array}{l}\text { Azimuth } \\
\left(\left(^{\circ}\right)\right.\end{array}$ & $\begin{array}{c}\text { Plunge } \\
\left({ }^{\circ}\right)\end{array}$ & & & \\
\hline$\sigma 1$ & 155 & 15 & & & \\
\hline$\sigma 2$ & 63 & 6 & & & \\
\hline$\sigma 3$ & 312 & 74 & & & \\
\hline \multicolumn{3}{|c|}{ Optimally oriented faults } & $\begin{array}{c}\text { Strike } \\
\left({ }^{\circ}\right)\end{array}$ & $\begin{array}{l}\text { Dip } \\
\left({ }^{\circ}\right)\end{array}$ & $\begin{array}{c}\text { Rake } \\
\left({ }^{\circ}\right)\end{array}$ \\
\hline \multicolumn{3}{|c|}{$\begin{array}{l}1 \text { (lower Mohr's semi- } \\
\text { circle) }\end{array}$} & 264 & 16 & 111 \\
\hline \multicolumn{3}{|c|}{$\begin{array}{l}2 \text { (upper Mohr's semi- } \\
\text { circle) }\end{array}$} & 57 & 44 & 82 \\
\hline
\end{tabular}

When comparing this local stress with the regional estimate (blue line in the inset of Figure 3.8 , striking at $\sim 135^{\circ}$ ), we arrive at conclusion that the $\sigma_{1}$ axis (strike $\left.155^{\circ}\right)$ of the local field might be slightly rotated clockwise $\left(\sim 20^{\circ}\right)$ with respect the regional stress. The activated optimally oriented fault, striking at $264^{\circ}$, deviates by $\sim 44^{\circ}$ with respect to the main trend of the Transbrazilian Lineament (TBL). Note that future events in the same region might, in principle, activate also the second optimally oriented fault (strike $57^{\circ}$, dip $44^{\circ}$ ), which is closer to the TBL trend. 

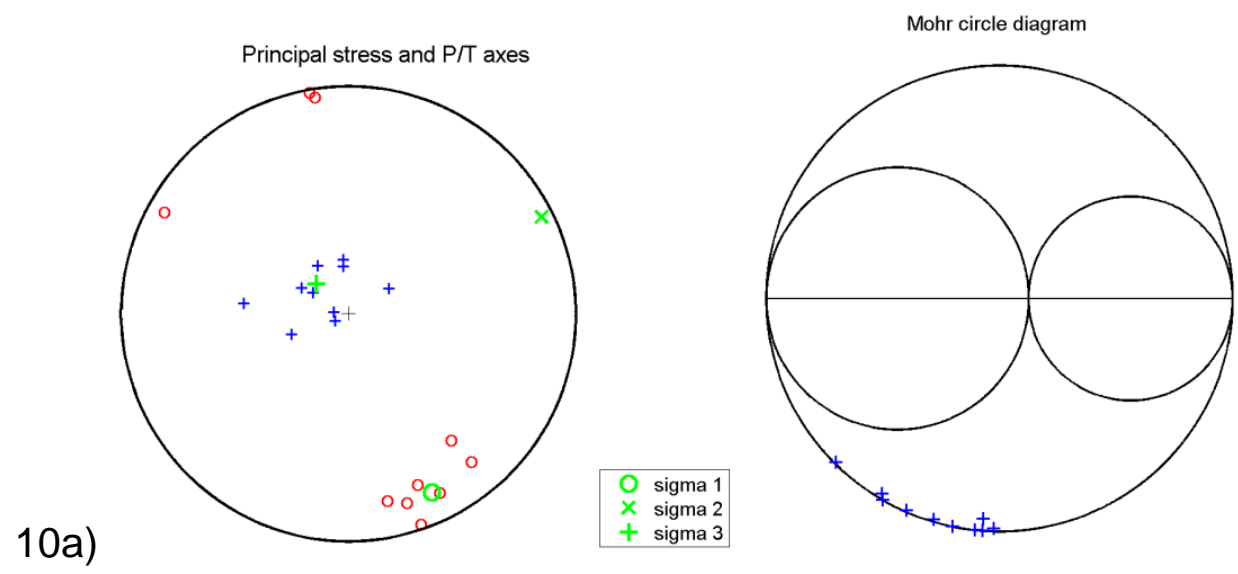

10b)

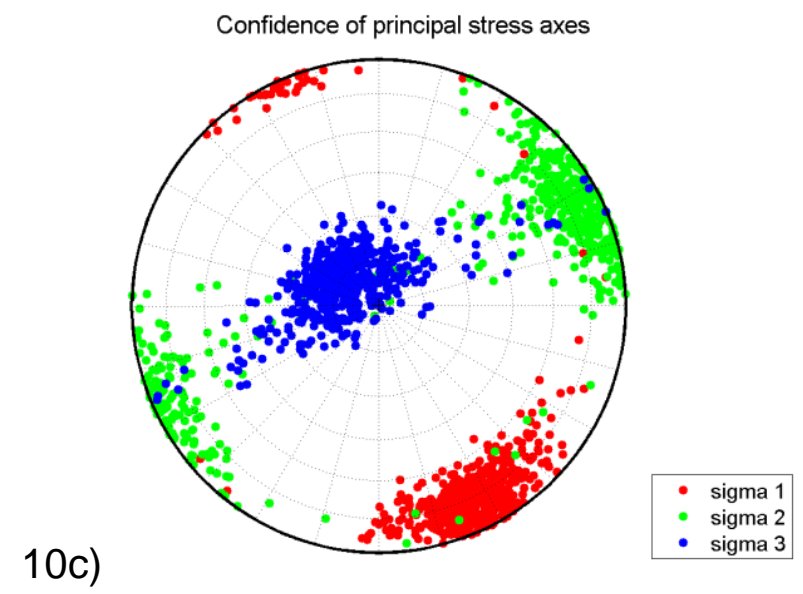

Figure 3.10 - Stress inversion based on focal mechanisms of Events 1 to 10. (a) P (red) \& $T$ (blue) axes and the stress directions $\sigma 1, \sigma 2$ and $\sigma 3$ (green). (b) Mohr's circle with the events shown by blue crosses. (c) Relative uncertainty of the stress axes resulting from random perturbation of the input data

\subsection{Discussion and conclusions}

Two aims of this paper were formulated in the Introduction, i.e. (i) to investigate focal mechanisms of Mara Rosa aftershocks, and (ii) to make inferences about local stress field. These two goals were achieved. A specific innovative feature of this study was a non-standard approach based on various quality criteria applied both to the input and output data in order to maximize credibility of the results. Careful relocation of the sequence, and thorough data inspection and selection resulted in 11 events, for which the moment magnitudes and reliable focal mechanisms (including relative uncertainty estimates) were obtained by means of the waveform inversion method. The results provide encouragement for similar investigations of weak events in 
Brazil and/or in other intraplate regions of low seismicity where as many events as possible must be deeply investigated. ISOLA software in its most recent version, plus customized quality criteria proved to be helpful. The main results are summarized in Table 3.3 and Figures 3.6-3.8. Most earthquakes (except Event 11) have similar focal mechanisms which are in agreement with the aftershock geometry and identify an activated reverse dip fault dipping to NNW.

The number of the studied events is small, thus only a rough estimate of the local stress field is possible. Nevertheless, the directions of principal stress axes were well determined (Table 3.4 and Figures $3.10 \mathrm{a}$ and $3.10 \mathrm{c}$ ). The study also indicated that from two optimally oriented faults (Table 3.4), existing in the given stress field, only one was activated during the Mara Rosa sequence (strike $264^{\circ}$ ). The second one (strike $57^{\circ}$ ), closer to the general trend of the Trans-Brazilian Lineament, but having probably a somewhat larger dip, may activate during future events in the studied region.

\section{Acknowledgments}

The authors thanks, Dr. V. Vavryčuk for significant help in using his STRESSINVERE code, Dr. Hans Agurto Detzel for providing the script to plot the P\&T axis. One of the authors (JZ) has been supported from the Czech Science Foundation grant GACR-14-04372S.

\subsection{References}

Agurto-Detzel, H., Assumpcao, M., Ciardelli, C., Albuquerque, D. F., Barros, L. V., \& Franca, G. S. L. (2014). The 2012-2013 Montes Claros earthquake series in the Sao Francisco Craton, Brazil: new evidence for non-uniform intraplate stresses in mid-plate South America. Geophysical Journal International, 200(1), 216-226. doi:10.1093/gji/ggu333

Angelier, J. (2002). Inversion of earthquake focal mechanisms to obtain the seismotectonic stress IV-a new method free of choice among nodal planes. Geophysical Journal International, 150(3), 588-609. doi:10.1046/j.1365-246X.2002.01713.x

Assumpção, M. (1992). The Regional Intraplate Stress-Field in South-America. Journal of Geophysical Research-Solid Earth, 97, 11889-11903. Retrieved from <Go to ISI>://A1992JG86200013

Assumpção, M. (1998a). Focal mechanisms of small earthquakes in the southeastern Brazilian shield: a test of stress models of the South American plate. Geophysical Journal International, 133, 490-498. doi:10.1046/j.1365-246X.1998.00532.x 
Assumpção, M. (1998b). Sismotectónica y esfuerzos en Brasil. In Física de la Tierra (Vol. 0). Retrieved from http://revistas.ucm.es/index.php/FITE/article/view/FITE9898110149A

Assumpção, M., Lima, T. M., \& Tomás, L. A. (1986). O sismo de Araguapaz de 14.01.1986 e o Lineamento Transbrasiliano. In 340 Congresso Brasileiro de Geologia, Goiânia (p. 5p).

Assumpcao, M., \& Suarez, G. (1988). Source mechanisms of moderate-size earthquakes and stress orientation in mid-plate South America. Geophysical Journal International, 92(2), 253-267. doi:10.1111/j.1365-246X.1988.tb01138.x

Assumpção, M., Schimmel, M., Escalante, C., Roberto Barbosa, J., Rocha, M., \& Barros, L. V. (2004). Intraplate seismicity in SE Brazil: stress concentration in lithospheric thin spots. Geophysical Journal International, 159(1), 390-399. doi:10.1111/j.1365246X.2004.02357.x

Assumpção, M., \& Sacek, V. (2013). Intra-plate seismicity and flexural stresses in central Brazil. Geophysical Research Letters, 40(3), 487-491. doi:10.1002/grl.50142

Assumpção, M., Dias, F. L., \& Zevallos, I. (2014). Intraplate Stress Field in South America from Earthquake Focal Mechanisms. South American Earth Sci (Submited), 1-32.

Barros, L. V., Assumpção, M., Quintero, R., \& Caixeta, D. (2009). The intraplate Porto dos Gaúchos seismic zone in the Amazon craton - Brazil. Tectonophysics, 469(1-4), 37-47. doi:10.1016/j.tecto.2009.01.006

Barros, L. V., M. Assumpção, Chimpliganond, C. N., Carvalho, J. M., Huelsen, M. G. Von, Caixeta, D. F., ... Fontenele, D. P. (2014). The Mara Rosa 2010 GT-5 earthquake and its possible relationship with the continental-scale TransBrasiliano lineament,. J. S. Am. Earth Sci. (submitted).

Benetatos, C., Málek, J., \& Verga, F. (2012). Moment tensor inversion for two microearthquakes occurring inside the Háje gas storage facilities, Czech Republic. Journal of Seismology, 17(2), 557-577. doi:10.1007/s10950-012-9337-0

Bouchon, M. (1981). A simple method to calculate Green's functions for elastic layered media. Bulletin of The Seismological Society of America, 71, 959. Retrieved from http://bssa.geoscienceworld.org/cgi/content/abstract/71/4/959|nfile://localhost/Users/ andrewking/Documents/Papers/1981/Bouchon/B Seismol Soc Am 1981 Bouchon.pdflnpapers://8a7341e4-9daa-4cOd-bcf2-2d9b813974af/Paper/p6475

Bouchon, M. (2003). A Review of the Discrete Wavenumber Method. Pure and Applied Geophysics, 160(3), 445-465. doi:10.1007/PL00012545

Caproni, N., \& Armelin, J. . (1990). Instrumentacão das escavações subterrâneas da UHE Serra da Mesa,. In Simpósio sobre Instrumentação Geotécnica de Campo - SINGEO'90 Associação Brasileira de Geologia de Engenharia, São Paulo. (p. vol. 1 249-257).

Cesca, S., Heimann, S., Stammler, K., \& Dahm, T. (2010). Automated procedure for point and kinematic source inversion at regional distances. Journal of Geophysical Research, 115(B6), B06304. doi:10.1029/2009JB006450

Chimpliganond, C., Assumpção, M., Von Huelsen, M., \& França, G. S. (2010). The intracratonic Caraibas-Itacarambi earthquake of December 09, 2007 (4.9 mb), Minas Gerais State, 
Brazil. Tectonophysics, 480(1-4), 48-56. doi:10.1016/j.tecto.2009.09.016

Coblentz, D. D., \& Richardson, R. M. (1996). Analysis of the South American intraplate stress field. Journal of Geophysical Research, 101(B4), 8643. doi:10.1029/96JB00090

Coutant, O. (1990). Program of numerical simulation AXITRA. Laboratoire de Géophysique Interne et Tectonophysique Report, University of Joseph Fourie.

CPRM - Serviço Geológico do Brasil. (2004). Brazilian Geological Survey. Geological Map of Brazil, scale 1:1,000,000, Geographic Information System, 2004. CD Rom.

Delouis, B., Charlety, C., \& Vallée, M. (2008). Fast determination of earthquake source parameters from strong motion records: Mw, fo- cal mechanism, and slip distribution,. In EGU General Assembly, Geophysical Research Abstracts 10, abstract 04939.

Dreger, D. S. (2003). TDMTINV-time-domain-seismic-moment-tensor-inversion - International Handbook of Earthquake and Engineering Seismology. International Geophysics (Vol. 81). Elsevier. doi:10.1016/S0074-6142(03)80290-5

Fernandes E., Blum M., \& Ribeiro, R. (1991). The Goiás seismic zone, a new approach. In Extended Abstract. $35^{\circ}$ Congress Braz. Geol. Soc., Salvador/BA (Vol. 2, pp. 553-558).

Ferreira, J. M., Oliveira, T., Takeya, M. K., \& Assumpção, M. (1998). Superposition of local and regional stresses in northeast Brazil: evidence from focal mechanisms around the Potiguar marginal basin. Geophysical Journal International, 134(2), 341-355. doi:10.1046/j.1365-246x.1998.00563.x

Fojtíková, L., Vavryčuk, V., Cipciar, A., \& Madarás, J. (2010). Focal mechanisms of microearthquakes in the Dobrá Voda seismoactive area in the Malé Karpaty Mts. (Little Carpathians), Slovakia. Tectonophysics, 492(1-4), 213-229. doi:10.1016/j.tecto.2010.06.007

Fojtíková, L., \& Zahradnik, J. (2014). A New Strategy for Weak Events in Sparse Networks: The First-Motion Polarity Solutions Constrained by Single-Station Waveform Inversion. Seismological Research Letters, 85(6), 1265-1274. doi:10.1785/0220140072

França, G. S. (2004). Estrutura da crosta no sudeste e centro-oeste do Brasil, usando função do receptor local: São Paulo. Revista Brasileira de Geofísica, 22(2), 143f. doi:10.1590/S0102$261 \times 2004000200007$

França, G. S., \& Assumpção, M. (2004). Crustal structure of the Ribeira fold belt, SE Brazil, derived from receiver functions. Journal of South American Earth Sciences, 16(8), 743-758. doi:10.1016/j.jsames.2003.12.002

Fuck, R. A. (1994). A Faixa Brasília e a Compartimentação Tectônica na Província Tocantins, IV Simpósio de Geologia do Centro-Oeste. SBG, Brasília, 184-187.

Fuck, R. A., Pimentel, M., \& Silva, L. (1994). Compartimentação Tectônica na Porção Oriental da Província Tocantins, IV Simpósio de Geologia do Centro-Oeste,. SBG , Brasília, 215-216.

Gephart, J. W., \& Forsyth, D. W. (1984). An improved method for determining the regional stress tensor using earthquake focal mechanism data: Application to the San Fernando Earthquake Sequence. Journal of Geophysical Research, 89(B11), 9305. doi:10.1029/JB089iB11p09305 
Havskov, J., \& Ottemöller, L. (2008). SEISAN: The Earthquake Analysis Software, Version 8.2. Bergen, Norway,: Inst. of Solid Earth Physics, Univ. of Bergen, Norway,.

Heidbach, O., Barth, A., Connolly, P., Fuchs, K., Müller, B., Tingay, M., ... Wenzel, F. (2004). Stress maps in a minute: The 2004 world stress map release. Eos, Transactions American Geophysical Union, 85(49), 521. doi:10.1029/2004EO490001

Kagan, Y. Y. (1991). 3-D rotation of double-couple earthquake sources. Geophysical Journal International, 106(3), 709-716. doi:10.1111/j.1365-246X.1991.tb06343.x

Krizova, D., Zahradnik, J., \& Kiratzi, A. (2013). Resolvability of Isotropic Component in Regional Seismic Moment Tensor Inversion. Bulletin of the Seismological Society of America, 103(4), 2460-2473. doi:10.1785/0120120097

Lienert, B. R. (1994). Hypocenter 3.2: A computer program for locating earthquakes locally, regionally and globally, $102 . \quad$ Retrieved from http://www.mendeley.com/research/hypocenter-32-computer-program-locatingearthquakes-locally-regionallyglobal-

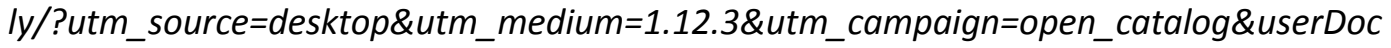
ument/d $=\{6 c c 7189 b-455 e-41 d 0-a 354-a 0 c 9 f 33 b c 8 d c\}$

Maercklin, N., Zollo, A., Orefice, A., Festa, G., Emolo, A., De Matteis, R., ... Bobbio, A. (2011). The Effectiveness of a Distant Accelerometer Array to Compute Seismic Source Parameters: The April 2009 L'Aquila Earthquake Case History. Bulletin of the Seismological Society of America, 101(1), 354-365. doi:10.1785/0120100124

Mendiguren, J. A., \& Richter, F. M. (1978). On the origin of compressional intraplate stresses in South America. Physics of the Earth and Planetary Interiors, 16(4), 318-326. doi:10.1016/0031-9201(78)90070-5

Michael, A. J. (1987). Use of focal mechanisms to determine stress: A control study. Journal of Geophysical Research, 92(B1), 357. doi:10.1029/JB092iB01p00357

Michele, M., Custodio, S., \& Emolo, A. (2014). Moment Tensor Resolution: Case Study of the Irpinia Seismic Network, Southern Italy. Bulletin of the Seismological Society of America, 104(3), 1348-1357. doi:10.1785/0120130177

Press, W. H., Teukolsky, S. A. ., Vetterling, W. T. ., \& Flannery, B. P. . (1997). Numerical Recipes in Fortran 77 The Art of Scientific Computing, Second Ed. (Cambridge University Press, Ed.) (p. 992).

Sen, A. T., Cesca, S., Bischoff, M., Meier, T., \& Dahm, T. (2013). Automated full moment tensor inversion of coal mining-induced seismicity. Geophysical Journal International, 195(2), 1267-1281. doi:10.1093/gji/ggt300

Soares, J. E., Berrocal, J., Fuck, R. a., Mooney, W. D., \& Ventura, D. B. R. (2006). Seismic characteristics of central Brazil crust and upper mantle: A deep seismic refraction study. Journal of Geophysical Research, 111(B12), B12302. doi:10.1029/2005JB003769

Sokos, E., \& Zahradnik, J. (2013). Evaluating Centroid-Moment-Tensor Uncertainty in the New Version of ISOLA Software. Seismological Research Letters, 84(4), 656-665. doi:10.1785/0220130002 
Sokos, E. N., \& Zahradnik, J. (2008). ISOLA a Fortran code and a Matlab GUI to perform multiple-point source inversion of seismic data. Computers \& Geosciences, 34(8), 967-977. doi:10.1016/j.cageo.2007.07.005

Triantafyllis, N., Sokos, E., Ilias, A., \& Zahradník, J. (2014). SCISOLA: Automatic moment tensor solution for SeisComp3. Seism. Res. Lett., Submitted.

Vackár, J., Burjánek, J., \& Zahradník, J. (2015). Automated detection of disturbances in seismic records; MouseTrap code. Seism. Res. Lett (accepted).

Vavryčuk, V. (2001). Inversion for parameters of tensile earthquakes. Journal of Geophysical Research, 106(B8), 16339. doi:10.1029/2001JB000372

Vavryčuk, V. (2014). Iterative joint inversion for stress and fault orientations from focal mechanisms. Geophysical Journal International, 199(1), 69-77. doi:10.1093/gji/ggu224

Vavryčuk, V., \& Kühn, D. (2012). Moment tensor inversion of waveforms: a two-step timefrequency approach. Geophysical Journal International, 190(3), 1761-1776. doi:10.1111/j.1365-246X.2012.05592.x

Waldhauser, F. (2001). HypoDD - A Program to Compute Double-Difference Hypocenter Locations. Menlo Park, California.: US Geological Survey.

Yagi Y. and Nishimura N. (2011). Moment tensor inversion of near source seismograms. Bull. Int. Indy. Sekem. Earthq. Engin. (Vol. 45, pp. 133-138).

Zahradnik, J., Serpetsidaki, A., Sokos, E., \& Tselentis, G.-A. (2005). Iterative Deconvolution of Regional Waveforms and a Double-Event Interpretation of the 2003 Lefkada Earthquake, Greece. Bulletin of the Seismological Society of America, 95(1), 159-172. doi:10.1785/0120040035

Zahradnik, J., \& Plesinger, A. (2005). Long-Period Pulses in Broadband Records of Near Earthquakes. Bulletin of the Seismological Society of America, 95(5), 1928-1939. doi:10.1785/0120040210

Zahradník, J., Janský, J., \& Plicka, V. (2008). Detailed waveform inversion for moment tensors of M 4 events: Examples from the Corinth Gulf, Greece. Bull. Seismol. Soc. Am., 98, 27562771.

Zahradnik, J., \& Plesinger, A. (2010). Toward Understanding Subtle Instrumentation Effects Associated with Weak Seismic Events in the Near Field. Bulletin of the Seismological Society of America, 100(1), 59-73. doi:10.1785/0120090087

Zahradnik, J., \& Custodio, S. (2012). Moment Tensor Resolvability: Application to Southwest Iberia. Bulletin of the Seismological Society of America, 102(3), 1235-1254. doi:10.1785/0120110216

Zoback, M. (1992). First- and second-order patterns of stress in the lithosphere: The World Stress Map Project. Journal of Geophysical Research, 97(B8), 11703. doi:10.1029/92JB00132 


\section{CAPITULO 4 - INVERSAO DO EVENTO PRINCIPAL}

\subsection{Introdução}

Neste capítulo são apresentados os resultados da inversão de formas de ondas, realizada com o programa ISOLA, do evento principal de Mara Rosa, magnitude $5.0 \mathrm{~m}_{\mathrm{b}}$, intensidade $\mathrm{VI}(\mathrm{MM})$, de 8 de outubro de 2010 . Esses resultados já foram apresentados no artigo "The Mara Rosa 2010 GT-5 Earthquake and Its Possible Relationship with the Continental-Scale Transbrasiliano Lineament", com autoria de Lucas Barros, Marcelo Assumpção Cristiano Chimpliganond, Juraci M Carvalho, Mônica G. Von Huelsen, Daniel Caixeta, George S França, Diogo Albuquerque, Vinicius Ferreira e Darlan Fontenele. O trabalho foi submetido para publicação, em setembro, 2014, no Journal of South American Earth Sciences; Ref. Nu.: SAMES-D-14-00166R1.

Considerando, que a mesma metodologia de inversão foi aplicada para o caso de micro-tremores, no capítulo anterior iremos omitir informações já apresentadas.

\subsection{Dados utilizados}

$\mathrm{Na}$ inversão utilizaram-se dados de formas de ondas e de polaridades nas estações regionais CAN3, SSV2 e BDFB. Essas estações foram usadas por serem as mais próximas do evento: 125 km, 144 km e 241 km, respectivamente (Figura 4.1). Também foram usados dados de polaridades da onda $P$ nas estações SFA1, JAN7, MAN1 e IGA2, bem como os dados de polaridades das estações RET2, RET3, RET4, RET8 e RET9, pertencentes ao projeto de pesquisa Estudos sobre o Lineamento Transbrasiliano (Rede de Estudos Tectônicos-Petrobras), Coordenado pelo Prof. J. E. Soares, ao qual agradeço a gentileza pela cessão desses dados. Os dados das formas de ondas dessas estações não foram usados na inversão devido à saturação dos sinais. Entretanto, utilizaram-se os dados de polaridades para verificar a consistência da solução de mecanismo focal determinado na inversão, com base na distribuição das polaridades na esfera focal. 


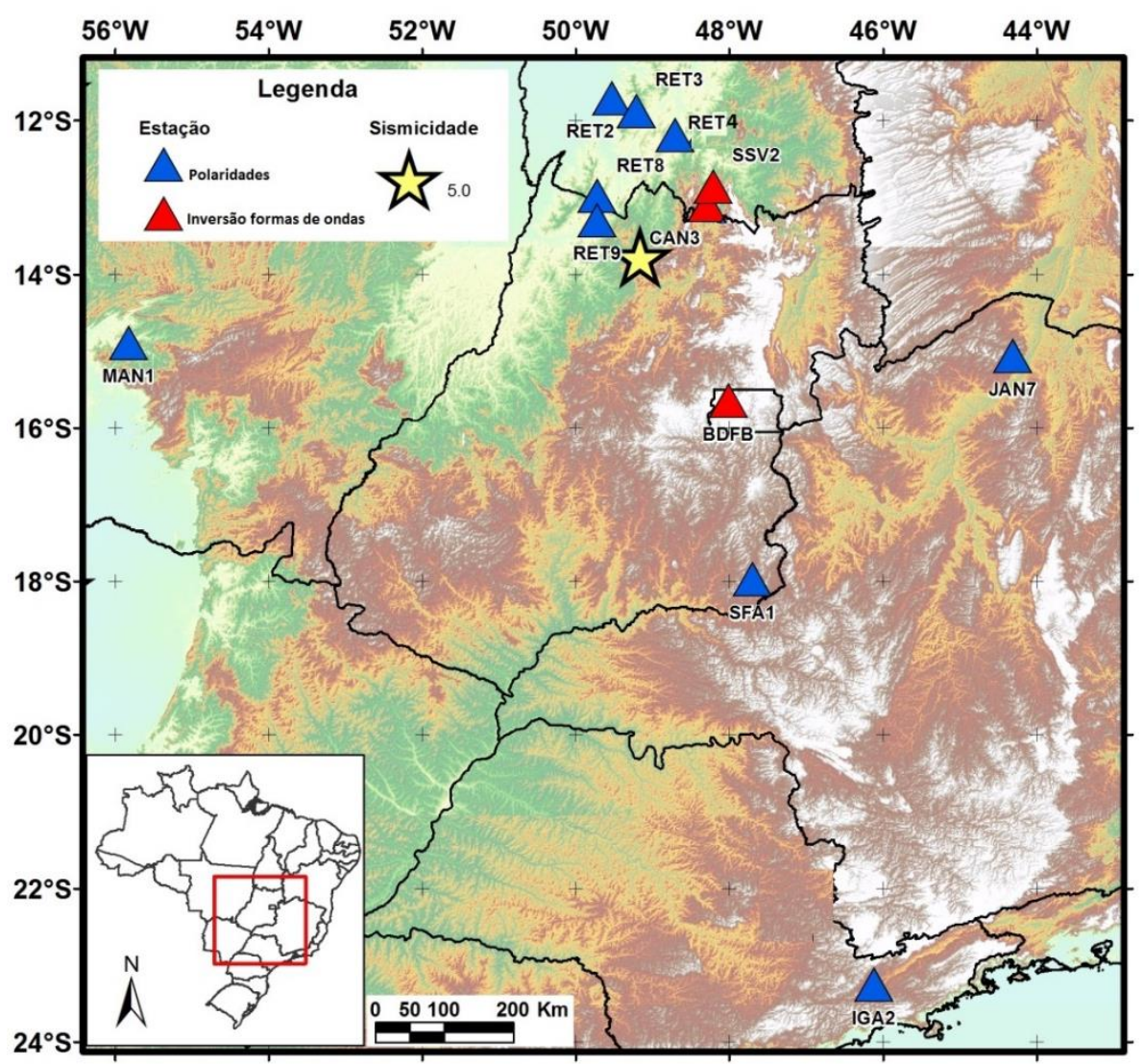

Figura 4.1 -Estações com formas de ondas utilizadas na inversão CAN3, SSV2 e BDFB (triângulos vermelhos) e estações usadas apesas as polaridades da fase (P) SFA1, JAN7, MANle RET2, 3, 4, 8 e 9 (triângulos azuis). A estrela denota o epicentro do evento principal de $M R$.

\subsection{Análise e resultados}

A inversão foi realizada como um modelo de fonte pontual e momento tensor desviatórico (deviatoric moment tensor), considerando que para eventos dessa magnitude não é recomendado a inversão com modelo de fonte multipontos. As funções de Green foram calculadas na banda de $0.05 \mathrm{a}$ $0.13 \mathrm{~Hz}$ e modelo crustal proposto por Barros et al. (2013) (Tabela 1).

Tabela 4.1 - Modelo de velocidades crustais utilizado na inversão.

\begin{tabular}{c|c|c|}
\hline $\begin{array}{c}\text { Depth of layer } \\
(\mathbf{k m})\end{array}$ & $\begin{array}{c}\text { Velocidade Vp } \\
(\mathbf{k m} / \mathbf{s})\end{array}$ & $\begin{array}{c}\text { Velocidade Vs } \\
(\mathbf{k m} / \mathbf{s})\end{array}$ \\
\hline 0 & 6.0 & 3.5 \\
\hline 12 & 6.6 & 3.8 \\
\hline 25 & 6.8 & 4.0 \\
\hline 35 & 7.2 & 4.2 \\
\hline 38 & 8.3 & 4.8 \\
\hline
\end{tabular}


Apesar das estações SSV2 e CAN3 utilizarem sismógrafos de período curto $(1 \mathrm{~Hz}-100 \mathrm{~Hz})$, foi possível observar a presença de sinais da fonte sísmica na banda de frequência utilizada na inversão $(0,05 \mathrm{~Hz}-0,13 \mathrm{~Hz})$, Figura 4.2.

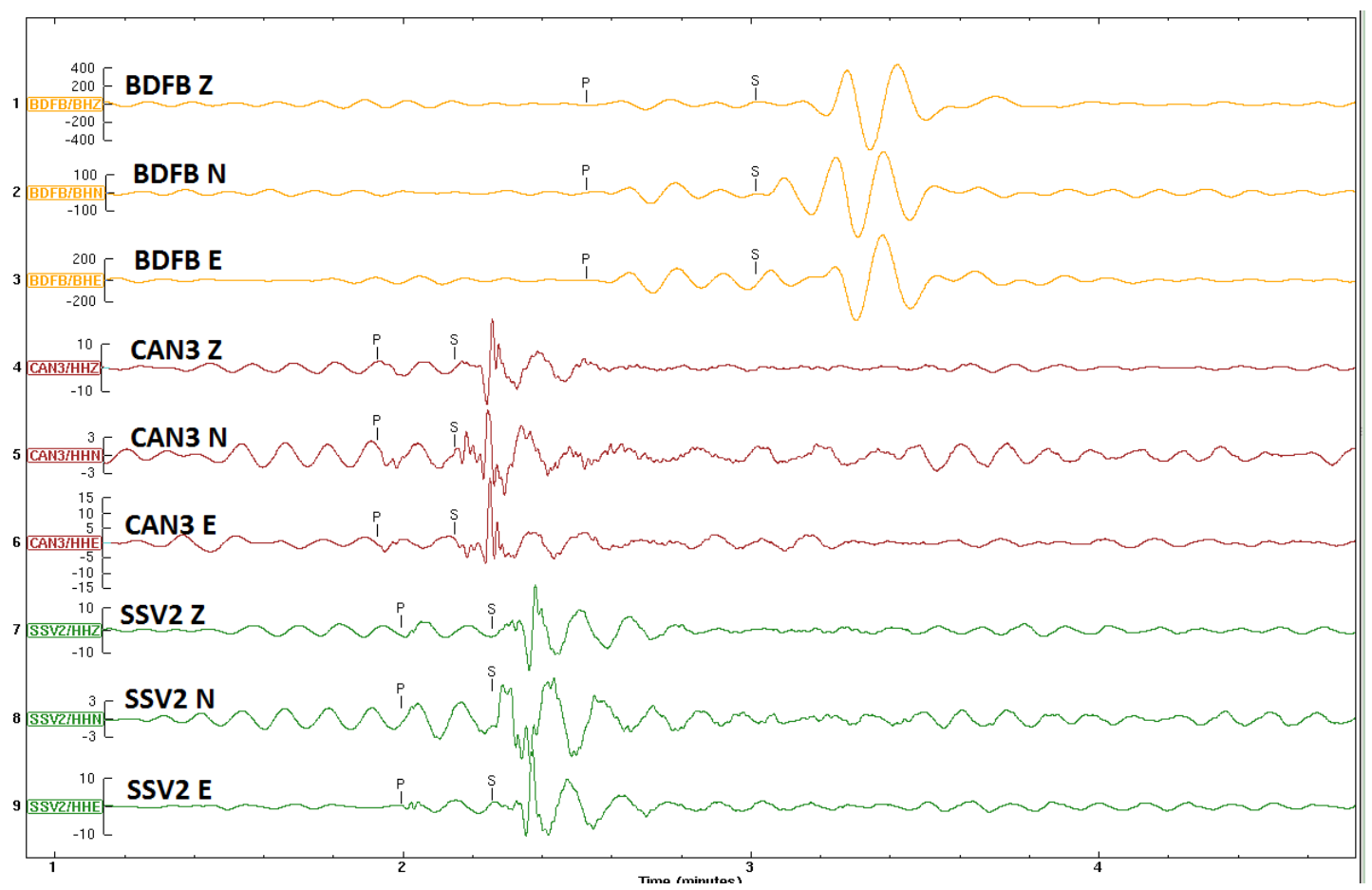

Figura 4.2 - Sinais no domínio do tempo das estações BDFB, CAN3 e SSV2 filtrados na banda de $0.05 \mathrm{~Hz}-0.13 \mathrm{~Hz}$. Observa-se claramente a existência de energia nos dados filtrados nas três estações. O instrumental da estação BDFB é de banda larga (120 sec - 50 $\mathrm{Hz})$.

A solução do momento tensor de melhor ajuste (entre sintétcos e observados) foi obtida por meio de busca em uma grade (grid search) com variação no espaço (profundidade) e no tempo (horizontal). A Figura 4.3 mostra o mecanismo focal que apresenta melhor correlação para a posição da fonte e o tempo de origem do centroide. A melhor correlação obtida foi para a fonte de número 4 (beachball vermelho), correspondendo a uma profundidade de 0,8 $\mathrm{km}$, um tempo de 200 milisegundos após o tempo de origem e um S/D/R (221/49/62). No entanto, considerando outros fatores, como a porcentagem de double-couple ( $\mathrm{DC} \%=66)$ e o conhecimento prévio da profundidade do evento (1.3 $\mathrm{Km}$ ), adotou se a fonte de número 6 (círculo amarelo) com profundidade de 1,0 km. A Figura 4.4 mostra que a solução número 4 foi a que obteve um melhor ajuste, ou seja obteve melhor VR (seta azul) e a solução de número 6 (seta vermelha) foi a que obteve uma melhor porcentagem de DC\%. As duas soluções (fonte 4 e fonte 6 ) têm mecanismos focais semelhantes, diferindo em 
apenas alguns graus, o que pode ser observado pela estabilidade dos resultados em relação à profundidade.

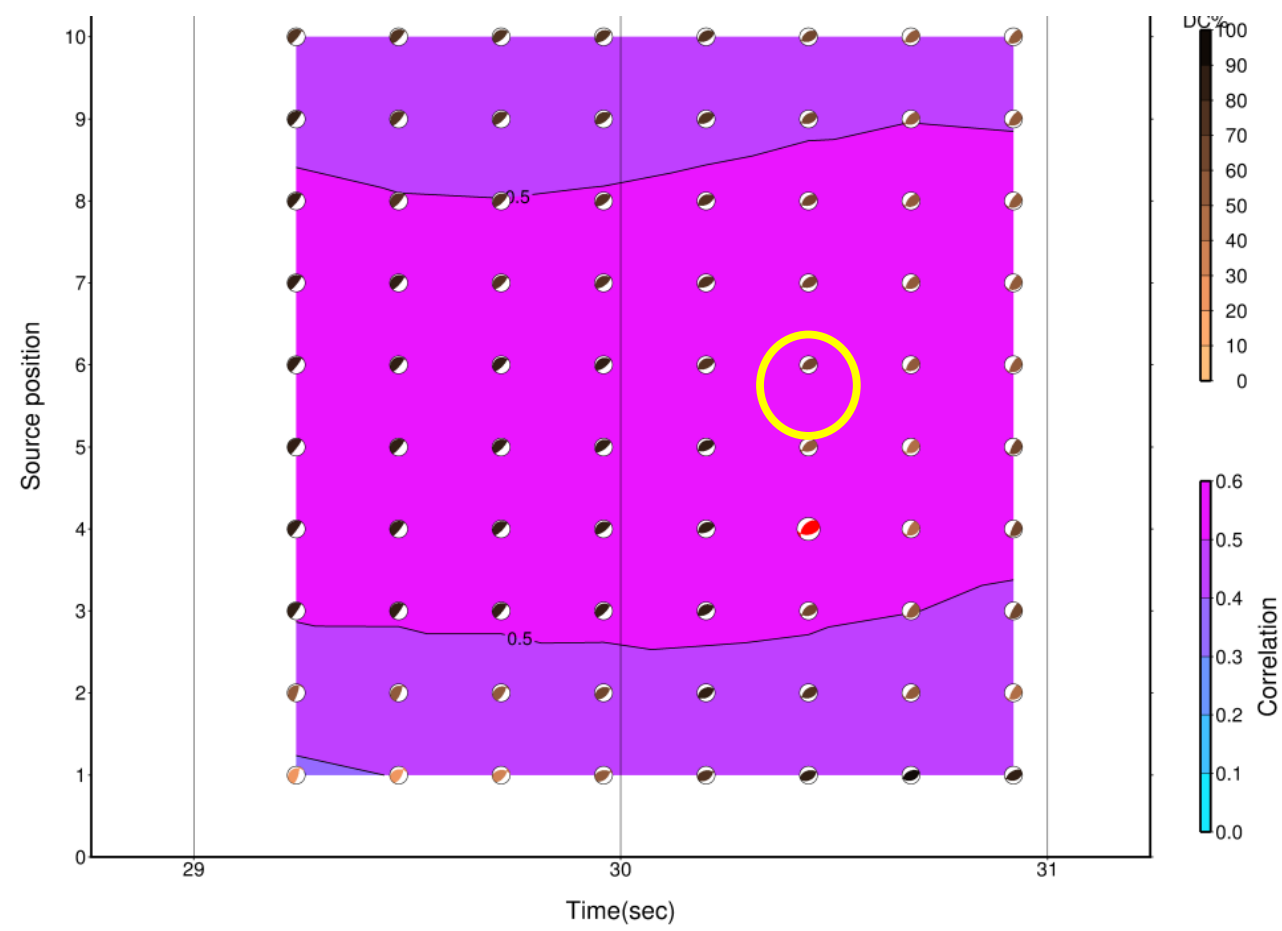

Figura 4.3 - correlação entre a posição da fonte (em profundidade) e o tempo de origem do centroide momento tensor. A maior correlação (beachball vermelho) foi obtida para a fonte número 4 (beachball vermelho). e a fonte adotada foi a de número 6 (círculo amarelo) com maior porcentagem de double-couple (DC\%).

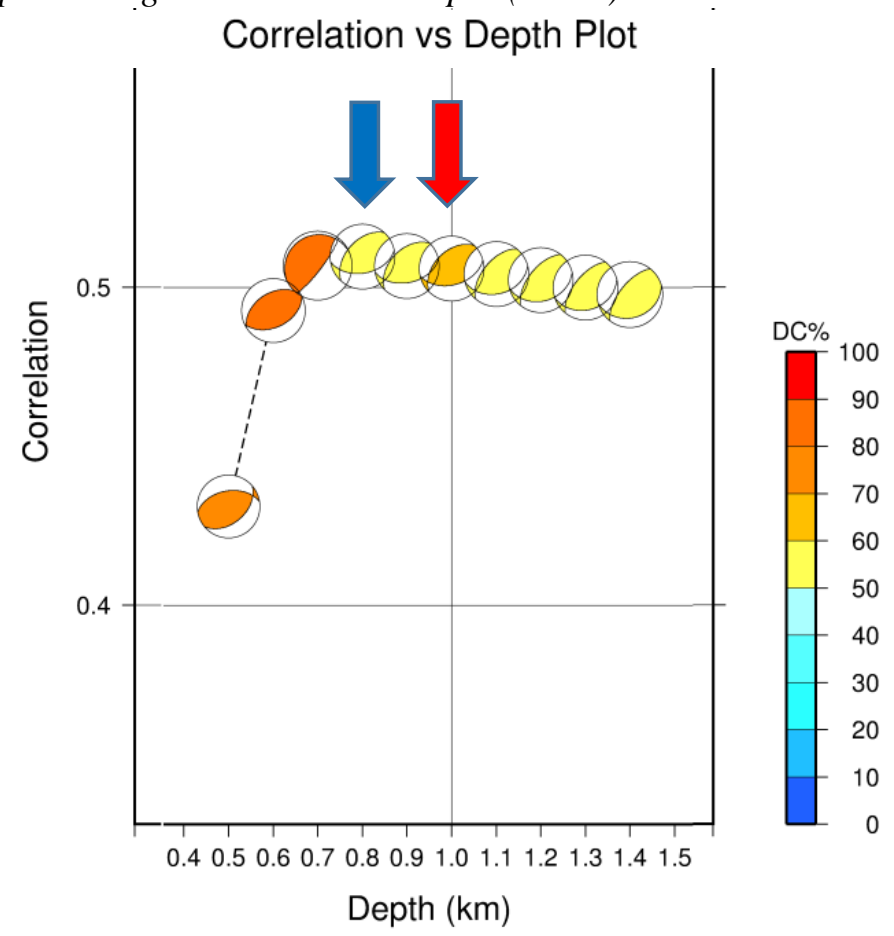

Figura 4.4 - Correlação dos mecanismos focais pela profundidade. A fonte 4 (seta azul) apresenta uma correlaçao ligeiramente maior que a fonte 6 (seta vermelha). Entretanto, a fonte 6 tem maior porcentagem de double-couple $(D C \%=66)$ e maior profundiade $(6 x$ $0.2 \mathrm{~km}=1.0 \mathrm{~km})$, mais próxima da profundidade do evento $\mathrm{GT5}(\mathrm{h}=1.3 \mathrm{~km})$. 
As correlações entre formas de ondas sintéticas (em vermelho) e observadas (em preto) nas estações CAN3, SSV2 e BDFB são indicadas na Figura 4.5. A falta de um bom ajuste pode ser atribuído às longas distâncias separando a fonte das estações sismográficas, a falta de precisão do modelo crustal adotado, à baixa relação sinal ruído, principalmente na estação CAN, bem como devido à geometria da rede. As três estações estão localizadas no mesmo lado da falha.

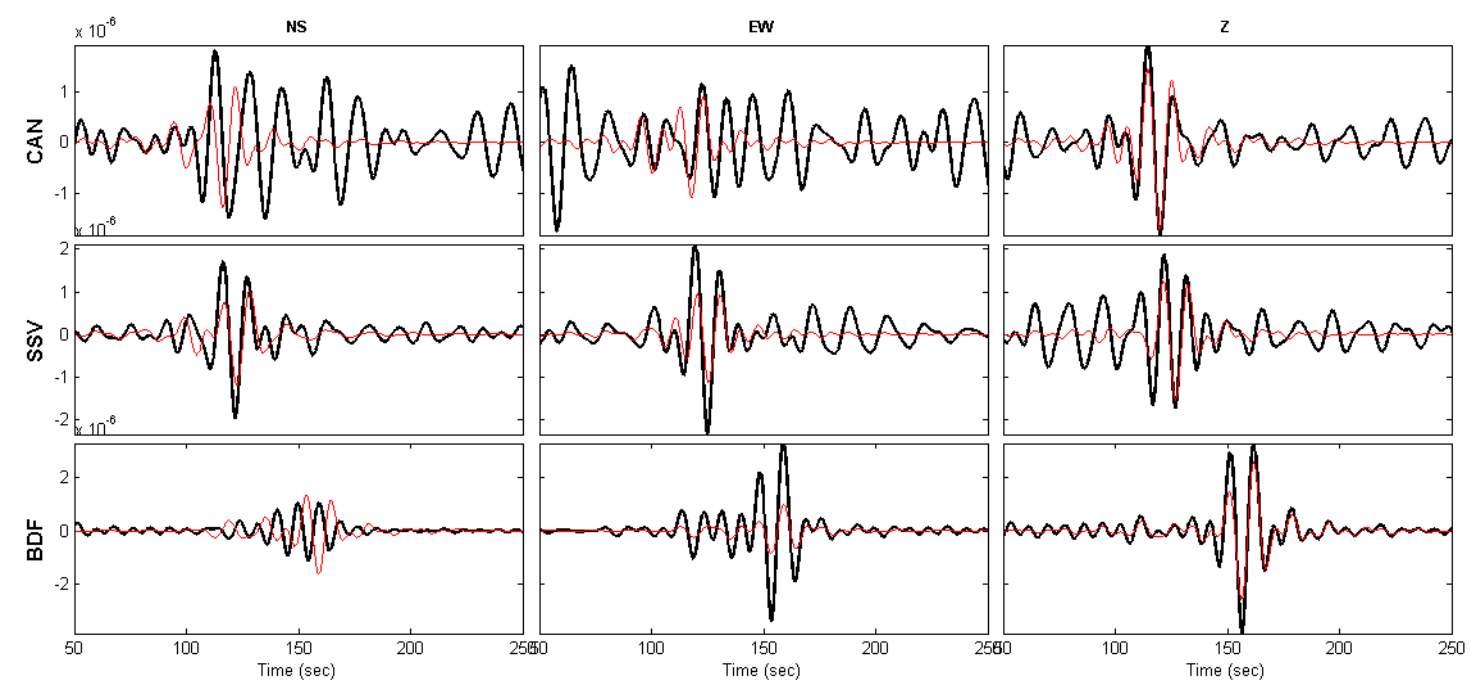

Figura 4.5 - Correlação entre sismogramas sintéticos (traços vermelhos) e observados (traços pretos) registrado em três estações regionais (CAN3, SSV2 e BDFB). A correlação das estações CAN3 (NS e EW) e BDFB (NS) são baixas nessas componentes devido principalmente às longas distâncias entre fonte e estações e baixa resolução do modelo crustal adotado.

Os parâmetros resultantes da inversão do evento principal de Mara Rosa, juntamente com aqueles obtidos por Barros et al. (2014) são apresentados na Tabela 4.2 e na Figura 4.6. O alto valor do Condition Number $(C N)$ indica que a configuração geográfica das estações em relação à fonte não é favorável à inversão. O valor da correlação, traduzido pela Variance Reduction (VR) da fonte com o melhor ajuste ficou relativamente baixo. No entanto, o resultado se mostrou estável em relação a variação da profundidade da fonte e concordante com o mecanismo focal composto, rotacionado em apenas 16으 graus medidos com método proposto por Kagan (1991) denominado aqui por " $K$-Angle". 


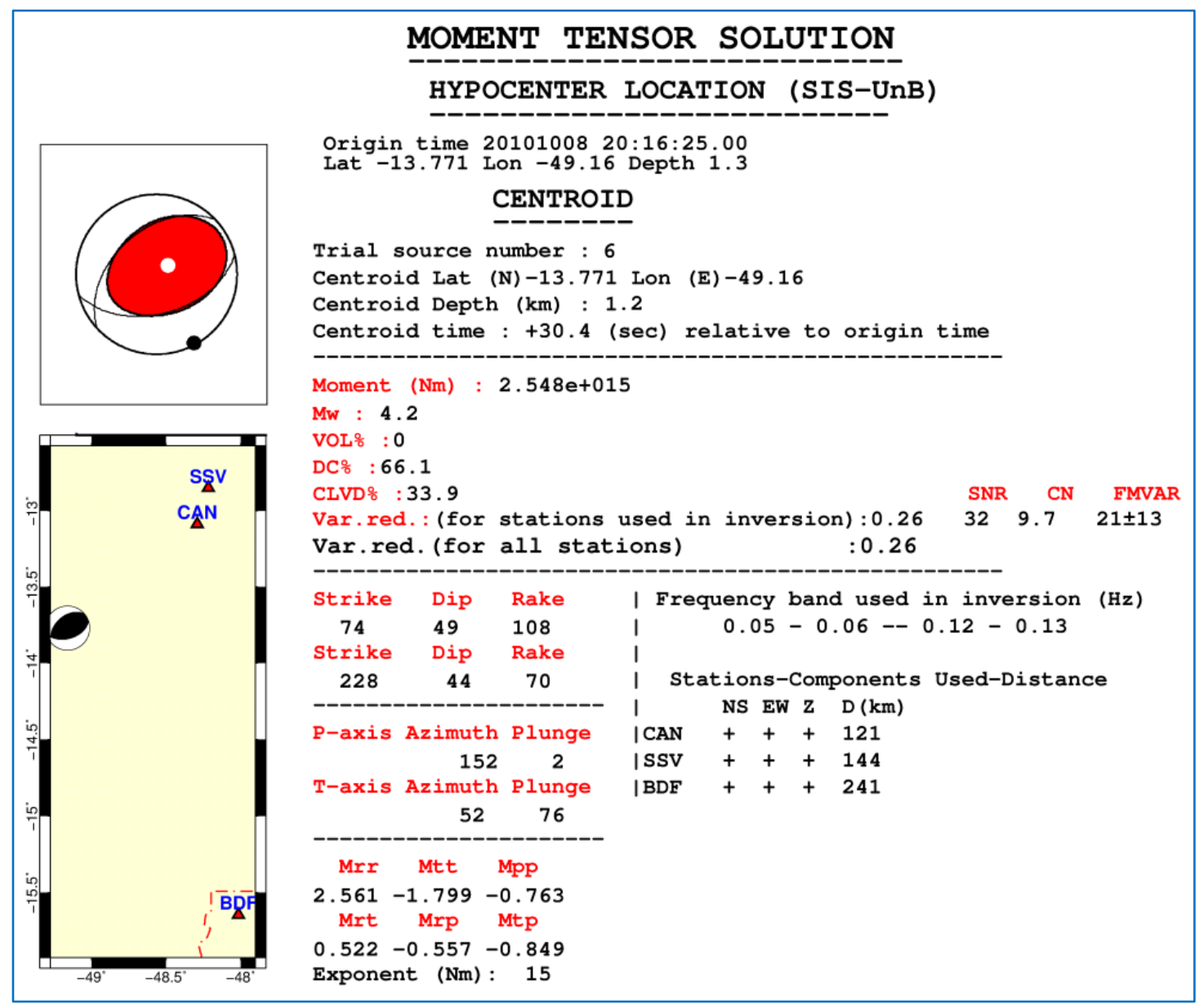

Figura 4.6 - resultados da inversão de formas de ondas do sismo principal de Mara Rosa, 8 Outubro 2010, magnitude $5.0 \mathrm{mb}$ e intensidade VI (MM). As estações utilizadas na inversão foram CAN3 (CAN), SSV2 (SSV) e BDFB (BDFB).

Tabela 4.2 - Resumo dos parâmetros da inversão com o pacote ISOLA. A primeira linha mostra os parâmetros do mecanismo focal composto e a segunda os parâmetros obtidos pela inversão.

\begin{tabular}{|l|l|l|r|r|r|r|r|r|r|r|}
\hline Stations & Freq. BandStrike/Dip/Rake & MW & $\begin{array}{c}\text { Dept } \\
\mathbf{h} \\
(\mathbf{K m})\end{array}$ & DC\% & SNR & VR & CN & K-Angle \\
\hline Referencia (MF composto) & $216 / 49 / 74$ & & 1.3 & \multicolumn{5}{|l|}{} \\
\hline
\end{tabular}

O mecanismo focal resultante da inversão foi validado pela concordância da maioria das polaridades das fases $(P)$ nas diversas estações sismográficas regionais e locais Figura 4.7. 


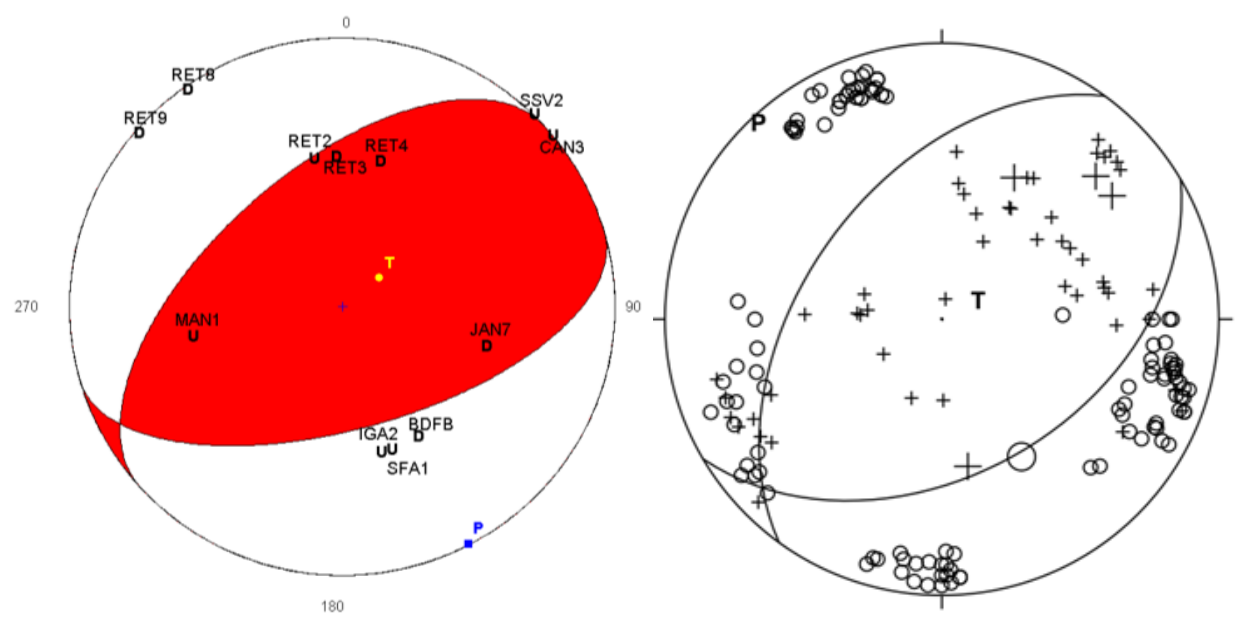

Figura 4.7 - a esquerda, mecanismo focal do evento principal de Mara Rosa obtido por inversão de formas de ondas, utilizando três estações regionais (CAN3, SSV2 e BDFB) $e$ polaridades das estações SSV2, CAN3, MAN1, IGA2, JAN7, SFA1, RET2, RET3, RET4, RET8, RET9 e BDFB. U (up) e D (down) são polaridades da primeira chegada da fase (P). A maioria das polaridades ou está em concordância ou próximas dos planos nodais do mecanismo focal resultante. À direita mecanismo focal composto obtido por Barros et. al., (2014) 


\subsection{Discussão e conclusões}

Apesar dos parâmetros da inversão, VR, DC\%, CN não se mostrarem ótimos, o mecanismo focal obtido é concordante com aquele obtido por Barros et al. (2014), com as polaridades das fases (P) em todas as estações e os eixos $P$ \& $T$ são compatíveis com o regime esforços regional (Assumpção, 1992a).

A magnitude de momento $\mathrm{Mw}=4.2$ obtida é compatível com a área de ruptura mapeada com a localização precisa das réplicas e com cálculo de magnitude de momento por análise espectral (Barros et al., 2014).

Alguns parâmetros da inversão estão a margem de valores ótimos. No entanto os resultados dos parâmetros de fonte obtidos são concordantes com estudos anteriores corroborando com a continuidade dos estudos na faixa sísmica Goiás-Tocantins.

\subsection{Referências}

Assumpção, M., 1992. The regional intraplate stress field in South America. Journal of Geophysical Research, 97(B8), p.11889. Available at: http://www.agu.org/pubs/crossref/ 1992.../91JB01590.shtml [Accessed December 23, 2014].

Barros, L. V. et al., 2014. The Mara Rosa 2010 GT-5 earthquake and its possible relationship with the continental-scale Transbrasiliano lineament,. J. S. Am. Earth Sci. (submetido).

Barros, L.V. et al., 2014. seismicic source's parameters of Brazilian earthquakes detected by Seismological Observatory network's in the last six years and relation between its body waves and seismic moment magnitudes. In Congresso de geologia, salvador - BA.

Barros, L.V., Assumpção, M. \& Carvalho, J.M., 2013. GT5 Earthquake Identified in Central Brazil. In CTBTO, ed. Comprehensive Nuclear-Test-Ban-Treaty Science and Technology. Vienna, pp. 17-21 Anexo C8.

Kagan, Y.Y., 1991. 3-D rotation of double-couple earthquake sources. Geophysical Journal International, 106(3), pp.709-716. Available at: http://gji.oxfordjournals.org/cgi/doi/10.1111/j.1365-246X.1991.tb06343.x [Accessed December 23, 2014]. 


\section{CAPÍTULO 5 - DISCUSSÃO E CONCLUSÕES}

\subsection{Discussão}

Nesta dissertação de mestrado foram realizadas inversões de formas de ondas do evento principal de Mara Rosa, de 08/10/2010 e de onze de suas réplicas (microtremores). Para assegurar a confiabilidades dos resultados, foram estabelecidos parâmetros de controle de qualidade, e por último foi feita a inversão dos mecanismos focais das réplicas.

Os resultados obtidos foram apresentados em dois artigos "Focal mechanisms and moment magnitudes of micro-earthquakes in central Brazil by waveform inversion with quality assessment and inference of the local stress field' (Capítulos 3), e na seção da inversão das formas de ondas do evento principal de Mara Rosa no artigo "The Mara Rosa 2010 GT-5 Earthquake and Its Possible Relationship with the Continental-Scale Transbrasiliano Lineament' (Capítulo 4). Esses dois artigos foram submetidos ao Jornal of South American Earth Science, em dezembro e setembro de 2014, respectivamente (primeiras páginas no Anexo I).

A zona sísmica Goiás Tocantins, onde está inserida a área de estudo, é caracterizada por sismos de baixas magnitudes $(<3.5)$, que associado à baixa densidade de estações sismográficas, torna difícil o estudo de mecanismos focais pelo método tradicional por polaridades.

O momento tensor do evento principal de Mara Rosa foi obtido pela inversão de formas de ondas em três estações regionais usando o modelo crustal (1D) derivado do trabalho de Barros et al. (2013). O mecanismo focal resultante foi validado com a utilização de 12 polaridades de estações regionais (Figura 4.7). O mecanismo focal e a magnitude de momento ( $\mathrm{Mw}$ ) são concordantes com estudos prévios de Barros et al. (2014). 
$\mathrm{Na}$ inversão de formas de ondas das réplicas, por se tratar de micro tremores, foi adotada uma abordagem mais criteriosa, utilizando parâmetros de controle de qualidade, tais como: inspeção cuidadosa dos dados de entrada; monitoramento dos parâmetros de inversão e validação dos resultados obtidos. A análise dos parâmetros de qualidade apresentados na Tabela 3.3 e nas figuras 3.6, 3.7 e 3.8 indica que os resultados da inversão dos onze microtremores são confiáveis.

Apesar da análise dos parâmetros de qualidade da inversão do evento 11 indicar que o resultado é confiável, o k-ângulo mostrou uma discrepância de $61^{\circ}$ graus em relação ao plano de falha do evento principal. Este estudo não encontrou uma explicação para essa diferença. Entretanto, isso pode ser um indicativo de que o evento 11 pode estar associado uma falha secundária.

Apesar do número de eventos usados na inversão dos mecanismos focais ser considerado baixo (Vavryčuk, 2014), o que teoricamente possibilitaria apenas uma estimativa aproximada do regime de esforços local, o resultado obtido (Tabela 3.4 e Figura 3.10) é consistente com as direções dos esforços determinados por Assumpção et al. (2008). O estudo também indicou que das duas falhas "idealmente orientadas" (Tabela 3.4) para campo de esforço encontrado, apenas uma foi ativada durante a sequência de Mara Rosa (strike $264^{\circ}$ ). A segunda (strike $57^{\circ}$ ), mais próxima da orientação do Lineamento Transbrasiliano, pode ser ativada em eventos futuros na área.

\subsection{Conclusões}

Os resultados da inversão do evento principal são concordantes com aqueles obtidos com o método tradicional de polaridades, portanto, tornando a solução do momento tensor a mais confiável.

A inversão dos microtremores teve resultados satisfatórios, podendo ser aplicada em outras áreas, e até mesmo servir de referência para o estudo de eventos de pequenas magnitudes com a inversão de suas réplicas. 
A inversão de mecanismos focais de réplicas mostrou que é possível obter o regime de esforços locais. No entanto, este tópico requer mais estudos futuros. Para aumentar a população de mecanismos focais na região e possibilitar um melhor estudo dos esforços é interessante a instalação de novas estações sismográficas na parte central da faixa sísmica.

\subsection{Referências}

Assumpção, M. et al., 2008. Intraplate Stress Patterns in Brazil with Recent Focal Mechanism Studies: Predominance of Mid-Continent compressional stresses and flexural effects at the continental margin. In AGU Joint Assembly. Fort Lauderdale, pp. CDROM, 1p.

Barros, L. V. et al., 2014. The Mara Rosa 2010 GT-5 earthquake and its possible relationship with the continental-scale Transbrasiliano lineament,. J. S. Am. Earth Sci. (submetido).

Barros, L.V., Assumpção, M. \& Carvalho, J.M., 2013. GT5 Earthquake Identified in Central Brazil. In CTBTO, ed. Comprehensive Nuclear-Test-Ban-Treaty Science and Technology. Vienna, pp. 17-21 Anexo C8.

Vavryčuk, V., 2014. Iterative joint inversion for stress and fault orientations from focal mechanisms. Geophysical Journal International, 199(1), pp.69-77. Available at: http://gji.oxfordjournals.org/cgi/doi/10.1093/gji/ggu224 [Accessed November 22, 2014]. 


\section{Anexo I}

Trabalhos produzidos no âmbito desta dissertação

Artigos para revistas com corpo editorial:

1) Focal mechanisms and moment magnitudes of micro- earthquakes in central Brazil by waveform inversion with quality assessment and inference of the local stress field

Authors: Carvalho J.M.; Lucas Barros; Jiri Zahradník

Journal of South American Earth Sciences (Elsevier Editorial System)

Ref. No.: SAMES-D-14-00227

Submetido: Dezembro/2014

2) The Mara Rosa 2010 GT-5 Earthquake and Its Possible Relationship with the Continental-Scale Transbrasiliano Lineament

Authors: Lucas Vieira Barros, Dr; Marcelo S Assumpção, Dr.; Cristiano Chimpliganond', Dr.; Carvalho J.M.; Mônica G Von Huelsen, Dr.; Daniel F

Caixeta; George S França, Dr.; Diogo F Albuquerque; Vinicius M Ferreira; Darlan P Fontenele

Journal of South American Earth Sciences (Elsevier Editorial System)

Ref. No.: SAMES-D-14-00166R2

Submetido: Setembro/2014

\section{Resumos expandidos:}

3) Carvalho J.M. and Barros, L.V. 2013. Seismic source parameters of local micro earthquake in Goiás State Brazil by waveform inversion. 13th Int. Cong. of the Brazilian Geophysical Society, Rio de Janeiro August, 2013

\section{Resumos publicados em Anais de Congressos}

4) Carvalho J.M., Barros, L. V., Zahradník, J, 2014. Seismic source parameters of local micro-earthquakes in Goiás State Brazil by waveform inversion. EARTH SCIENCES RESEARCH JOURNAL Abstracts 3th Congress of Seismology, Bogotá, Colombia 23 - 25 July 2014. 


\section{Elsevier Editorial System(tm) for Journal of South American Earth Sciences} Manuscript Draft

Manuscript Number:

Title: Focal mechanisms and moment magnitudes of micro- earthquakes in central Brazil by waveform inversion with quality assessment and inference of the local stress field

Article Type: SI: Moment Tensors, Stresses

Keywords: Waveform inversion

Focal mechanism

Micro-earthquakes

Tectonic stress field

Intraplate seismicity

Brazilian seismicity

Goias Tocantins Seismic Zone

TransBrazilian Lineament

Corresponding Author: Mr. Juraci Carvalho,

Corresponding Author's Institution:

First Author: Juraci Carvalho

Order of Authors: Juraci Carvalho; Lucas Barros; Jiri Zahradnik

Abstract: We investigated the ability of the full waveform inversion to retrieve focal mechanisms of micro-earthquakes with epicenters in Brazil. We demonstrate feasibility of the waveform inversion of weak events. The inversion was modeled at relatively high frequencies $(1.5-2.0 \mathrm{~Hz})$. The obtained solutions are in agreement with results from previous study and known fault geometry. The focal mechanisms inversion allowed an estimation of the local stress field. The paper may serve as a pilot study for similar investigations in intraplate regions.

Suggested Reviewers: Václav VAVRYČUK PhD

Faculty of Mathematics and Physics , Charles University in Prague, Czech Republic vv@ig.cas.cz

Dr. Vavrycuk has a vast knowledge in the paper research area and will provide a great contribution on the paper review.

Zaher Hossein Shomali PhD

Proffessor, Department of Earth Sciences, Geophysics; , Svenska nationella seismiska nätet hossein.shomali@geo.uu.se

Dr. Hossein Shomali is an expert in wave form inversion and has knowledege on the Brazilian seismicity.

Hans Agurto-Detzel PhD

Pos-PhD Student at the University of Sao Paulo, Institute of Astronomy, Geophysics and Atmospheric Sciences IAG, University of São Paulo 
Elsevier Editorial System(tm) for Journal of South American Earth Sciences Manuscript Draft

Manuscript Number:

Title: THE MARA ROSA 2010 GT-5 EARTHQUAKE AND ITS POSSIBLE RELATIONSHIP WITH THE CONTINENTAL-SCALE TRANSBRASILIANO LINEAMENT

Article Type: Research Paper

Keywords: Ground-truth (GT) events

TransBrasiliano Lineament

Mara Rosa earthquake

Goias-Tocantins Seismic Zone (GTSZ)

Focal mechanism solution

Waveform inversion

Moment-tensor

Relative locations

Cross-correlation analysis

Corresponding Author: Dr. Lucas Vieira Barros, Dr

Corresponding Author's Institution: University of Brasília

First Author: Lucas Vieira Barros, Dr

Order of Authors: Lucas Vieira Barros, Dr; Marcelo S Assumpção, Dr.; Cristiano Chimpliganond, Dr.; Juraci M Carvalho, MsC; Mônica G Von Huelsen, Dr.; Daniel F Caixeta; George S França, Dr.; Diogo F Albuquerque; Vinicius M Ferreira; Darlan P Fontenele

Abstract: On October 8th, 2010, a 5.0 mb earthquake with intensity VI (MM) occurred close to Mara Rosa, in the North of Goiás State, central Brazil, in an area where previous low magnitude seismicity had been observed. This earthquake was felt up to $300 \mathrm{~km}$ away from the epicenter, and was the biggest event ever detected in Central Brazil Seismic Zone. Despite the difficulty of associating earthquakes in Stable Continental Interior with geological structures, this event is possibly related to the reactivation of a geological fault of the continental-scale TransBrasiliano Lineament (TBL): the aftershock activity observed with an 8-station seismic network, indicates a NW dipping, SW-NE trending reverse fault, parallel to the TBL. The P axis is NW-SE oriented, consistent with expected stress direction in the region. $\mathrm{P}$ and $\mathrm{S}$ phases for the mainshock at regional stations were picked using waveform correlation with other reference events well recorded by both local and regional stations. The use of regional station corrections allowed the mainshock to be located with uncertainties small enough to qualify for a GT5 event, which will help to constrain 3D velocity models in South America. We found that the aftershocks were distributed around a circular area about 1.5 to $2.0 \mathrm{~km}$ across, with no events in the middle. This is interpreted as the mainshock rupture completely releasing all stresses. The rupture area and the mainshock moment correspond to a stress-drop of about $2 \mathrm{MPa}$.

Suggested Reviewers: Sergio Barrientos Dr.

University of Chile

sbarrien@dgf.uchile.cl

Dr. Barrientos is very experience with Ground Truth events.

Ronnie Quintero Dr.

Seismologist, OVSICORI-UNA - Costa Rica

ronnie.quintero.quintero@una.cr

Dr. Quintero is has a large expertize in earthquakes locations and seismic source parameter

determination, including waveform inversion.

Javier A Pacheco Dr.

OVSICORI-UNA - Costa Rica

javier.pacheco.alvarado@una.cr

Dr. Pacheco has being working with a wide range of subjects in seismology.

Stéphane Drouet Dr.

National Observatory-Science Technology Minister - Rio de Janeiro, Brazil

st.drouet@gmail.com

Dr. Drouet is engaged in understanding the intra-plate seismicity in Brazil. 


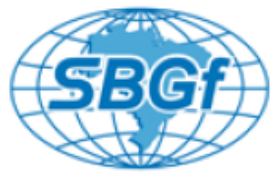

\title{
Seismic source parameters of local micro earthquake in Goiás State Brazil by waveform inversion
}

\begin{abstract}
Juraci Mário de Carvalho' ${ }^{1}$ and Lucas Vieira Barros ${ }^{1}$.
Copyright 2013, SBGf - Sociedade Brasileira de Geofisica

This paper was prepared for presentation during the 13 th international Congress of the Brazilian Geophysical Society held in Rio de Janeiro, Brazli, August 26-29, 2013. Contents of this paper were reviewed by the Technical Committee of the 13th Inteprestint any position of the SBGf, ts officers or members. Electronic reproduction of storage of any part of this paper for commercial purposes without the wnitten consent of the Brazilian Geophysical Society is prohibited.
\end{abstract}

1 Observatório Sismológico - UnB, Brasilia - DF, Brasil - juraci@unb.br and lucas@unb.br

\section{Abstract}

The waveform inversion of local seismic events with epicenter in Mara Rosa, in the Goiás State of Brazil was taken to obtain the moment tensor using the package ISOLA. The events are from an aftershock activity of 5.0 $\mathrm{mb}$ main shock and MMI VI in the range of 1.2 to $2.0 \mathrm{mD}$. The data was band-pass filtered in the frequency of 1.0 $1.45 \mathrm{~Hz}$. For preliminary results, satisfactory consistency was achieved when we compare the results reached in this work with those obtained by the polarity method.

\section{Introduction}

On October $8^{\text {th }}, 2010$ an earthquake of magnitude $5.0 \mathrm{mb}$ and intensity VI (MM) startled residents in the northern region of the Goiás state, having also been felt in Brasília and Goiânia which are located about $250 \mathrm{~km}$ and $300 \mathrm{~km}$ away, respectively. The aftershock activity of this event was studied with a local seismographic network of eight stations that detected more than 600 events of which 110 were located by five or more stations operating simultaneously (Barros et al. 2011). The network operated between October 2010 and June 2011. We selected the strongest events detected by eight stations simultaneously to determine the composite focal mechanism (Snoke, 1984). The nodal plane solution of a reverse faulting with strike of $214^{\circ}$, dip of $50^{\circ}$ and a rake of $74^{\circ}$ was obtained. The strikes and plunges of the axes of compression (P) and distension (T) are, respectively, $315^{\circ}$ and $58^{\circ}, 3^{\circ}$ and $78^{\circ}$. The aftershock epicenter distribution is in agreement with the fault orientation and both are in agreement with the Transbrasiliano Lineament (TL) (Barros et al. 2012)

In this work we present the preliminary results obtained with micro earthquakes waveforms inversion to obtain the moment tensor using the ISOLA package (Sokos and Zahradinik, 2008; Zahradinik et al. 2005).

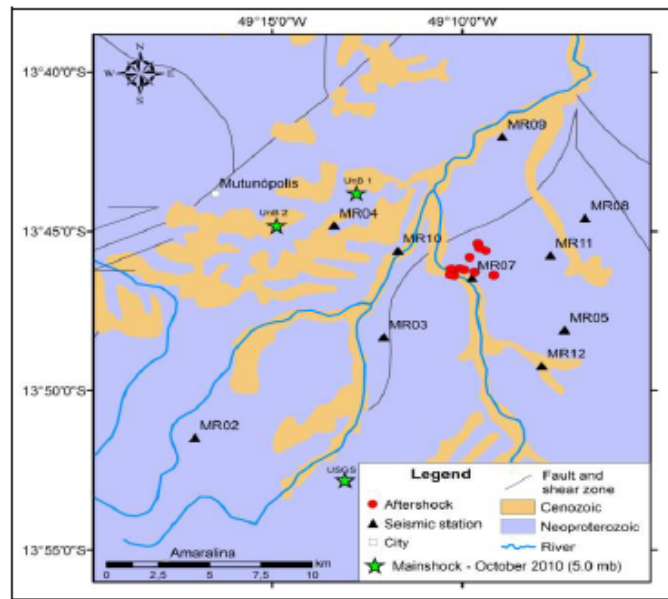

Figura 1. - Geological map of study area. Stars denote the mainshock epicenters determined by USGS and UnB, using the velocity models of Kwitko and Assumpção, 1990 (UnB1) and the NewBR model by Assumpção et. al. (2010) (UnB2). Red circles denote the best event epicenters (11) detected simultaneously by six or more local stations (black triangles)

\section{Data}

We selected for analysis 11 events detected by six or more stations and with a magnitude between 1.2 and 2.0 (Table 1). The locations were identified with the program Hypocenter (Lienert, 1994) in SEISAN (Havskov \& Ottemöller, 2009) using a two layer 1-D velocity model (Table 2). The RMS average of the travel time residuals are in the range of $30 \mathrm{~ms}$ and the depths are between $800 \mathrm{~m}$ and $1500 \mathrm{~m}$. Data were acquired with GURALP Manufacturer, Model 6TD (30 s - $50 \mathrm{~Hz}$ ) and DM24/CMG$40 \mathrm{~T} 1(1-100 \mathrm{~Hz})$ instruments in a sampled rate of 100 sps. Figure 2 shows the record of April $28^{\text {th }}, 2011$ event at four stations of the Mara Rosa network (event No. 11 in Table 1). 


\begin{tabular}{ccc}
\hline EARTH SCIENCES \\
RESEARCH JOURNAL \\
Earth Sci Res. J. Vol I8 Special Issue (July, 20I4)
\end{tabular}

\title{
Seismic source parameters of local micro-earthquakes in Goiás State Brazil by waveform inversion
}

\author{
Juraci Carvalho', Lucas Vieira Barros ${ }^{t}$, Jiři Zahradnik ${ }^{2}$ \\ 'Observatório Sismológico - UnB, Brasilia - DF, Brasil. \\ 'Faculty of Mathematics and Physics - Charles University in Prague, Czech Republic. \\ juraci@gmail.com
}

\section{ABSTRACT}

Only few earthquake focal mechanism studies have been done in Brazil due to low seismicity associated with low density of seismograph stations. Thus a little is known about intraplate tectonic stress distribution in Brazil and active faults, which is the fundamental information for understanding seismicity and making seismic hazard studies possible. To overcome the lack of such information, in addition to the traditional technique to obtain focal mechanism solutions by using the first P-phase polarities recorded in many stations, we demonstrate how to take advantage of full waveform inversion, from few stations. More specifically, in this contribution we perform the inversion of data from weak events, such as aftershocks, recorded by local stations (epicentral distance less than $8 \mathrm{~km}$ ). The unique feature of this work is that thanks to the short epicentral distances we are able to model the complete wavefield up to the relatively high frequencies of $2.0 \mathrm{~Hz}$.

We investigate the focal mechanisms using the ISOLA package developed by Sokos and Zahradnik $(2008,2013)$ for local and regional events, based on the least-squares calculation of the moment tensor and grid search of the centroid position and time. The Green function calculation is based on the discrete-wavenumber method developed by Countant (I990) and Bouchon (I98I, 2003). The events are aftershocks of a $5.0 \mathrm{mb}$ earthquake (MMI VI) which occurred on 20I0/I0/08 in Mara Rosa, Goiás State, Brazil. Eleven events recorded by at least four and up to seven stations from a temporary local seismographic network, using broad band ( $30 \mathrm{~s}$ to IOO $\mathrm{Hz}$ ) and short period (Is to $\mathrm{IOOHz}$ ) seismometers were used. The magnitudes range from I. 2 to $2.0 \mathrm{mD}$ and distances vary from $I .0 \mathrm{~km}$ to $8.0 \mathrm{~km}$. In a previous study, Barros et al. (20I2) showed a fault striking to NW-SE and dipping to NW. A local crustal model developed by Barros et al. (20I2) was used in this study.

To assess the results reliability, we used several quality-control parameters, such as VR (variance reduction), $\mathrm{CN}$ (condition number), $\mathrm{DC} \%$ (doublecouple percentage), SNR (signal-to-noise ratio), the first-motion polarity agreement, the focalmechanism uncertainty described by the Kagan-angle dispersion (Zahradnik and Custodio, 20I2), etc. Prior to the inversion a thorough data inspection of the waveforms was done to remove data with instrumental disturbances (the so-called 'mice'; Zahradnik and Plesinger, 2005 and 20I0), electronic noise, excessive cultural noise and/or any kind of equipment malfunction which could bias the inversion results.

The inversion frequency band was determined according to the SNR (the minimum usable frequency, f-low $=I .5 \mathrm{~Hz}$ ) and according the corner frequency and velocity model resolution (f-high = $2.0 \mathrm{~Hz}$ ). The grid-searched centroid positions (trial sources) were situated below the fixed epicenter in a depth range of about $3.8 \mathrm{~km}$ and in a time window of about $\pm 3.0 \mathrm{sec}$ around the origin time to allow optimization of the deviatoric moment tensor.

Good results were obtained for eight of the eleven studied events with most of the quality-control parameters meeting optimum values established in previous studies. The results are in general agreement with Barros et al. (20I2), but, importantly, they also 


\section{Anexo II}

\section{Formas de ondas (sintéticas e observadas) obtidas na inversão das onze réplicas}
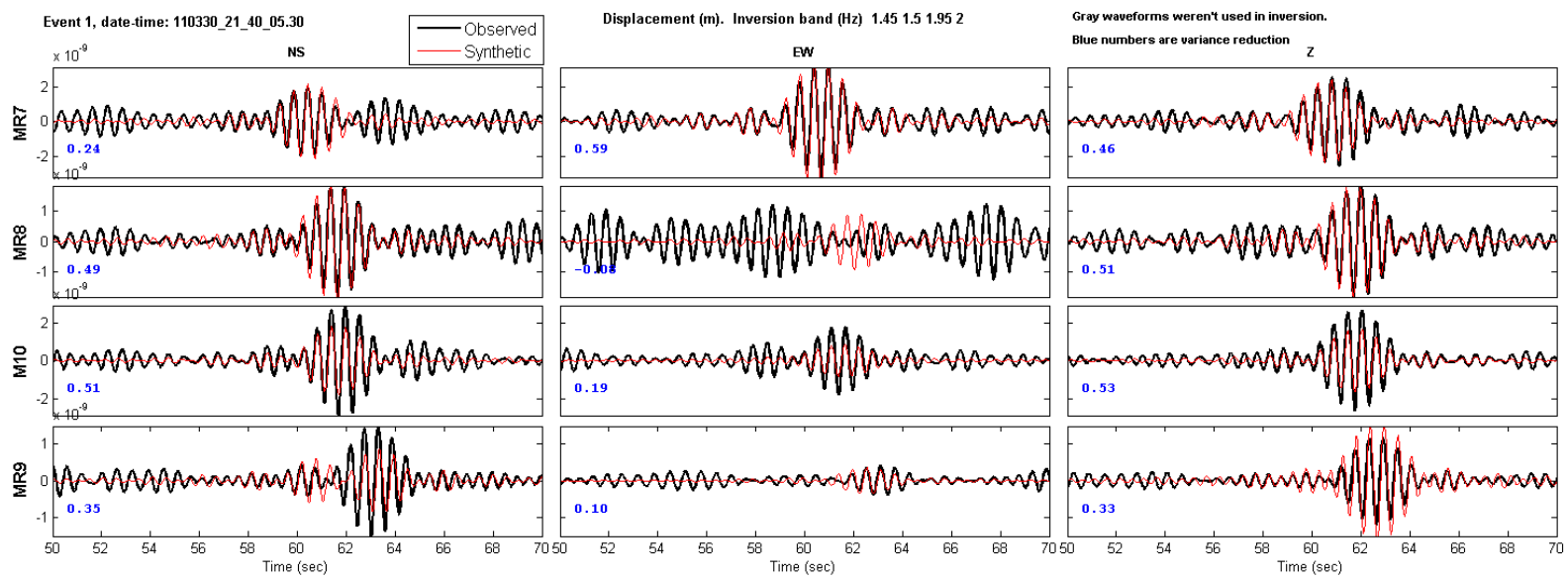

Figure II.1 - Comparison between the observed (black) and synthetic (red) waveforms. The plot refers to Event 1 of Tables 3.2 and 3.3. The waveform match is quantified by the variance reduction values per station components (blue numbers). The horizontal time axis is in seconds, $t=50$ sec corresponds to 10 seconds after the origin time. The vertical axis indicates the displacement amplitude in meters.
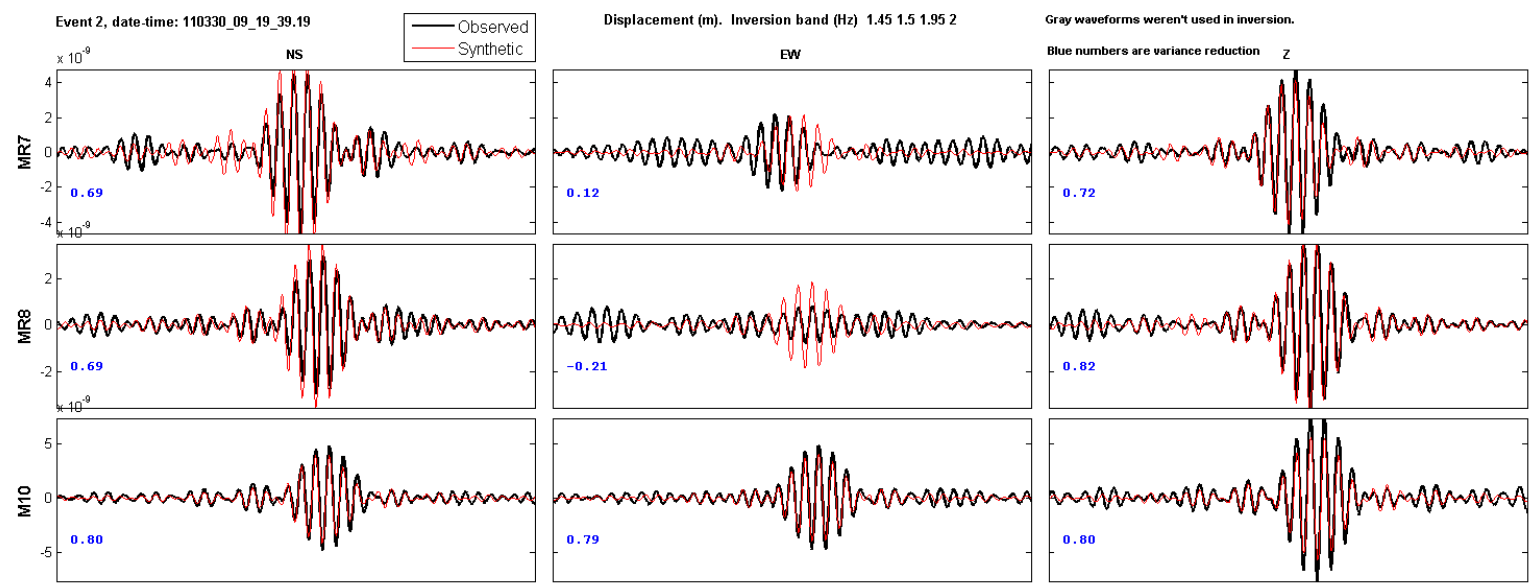

Figure II.2 - Comparison between the observed (black) and synthetic (red) waveforms. The plot refers to Event 2 of Tables 3.2 and 3.3. The waveform match is quantified by the variance reduction values per station components (blue numbers). The horizontal time axis is in seconds, $t=50 \mathrm{sec}$ corresponds to 10 seconds after the origin time. The vertical axis indicates the displacement amplitude in meters. 


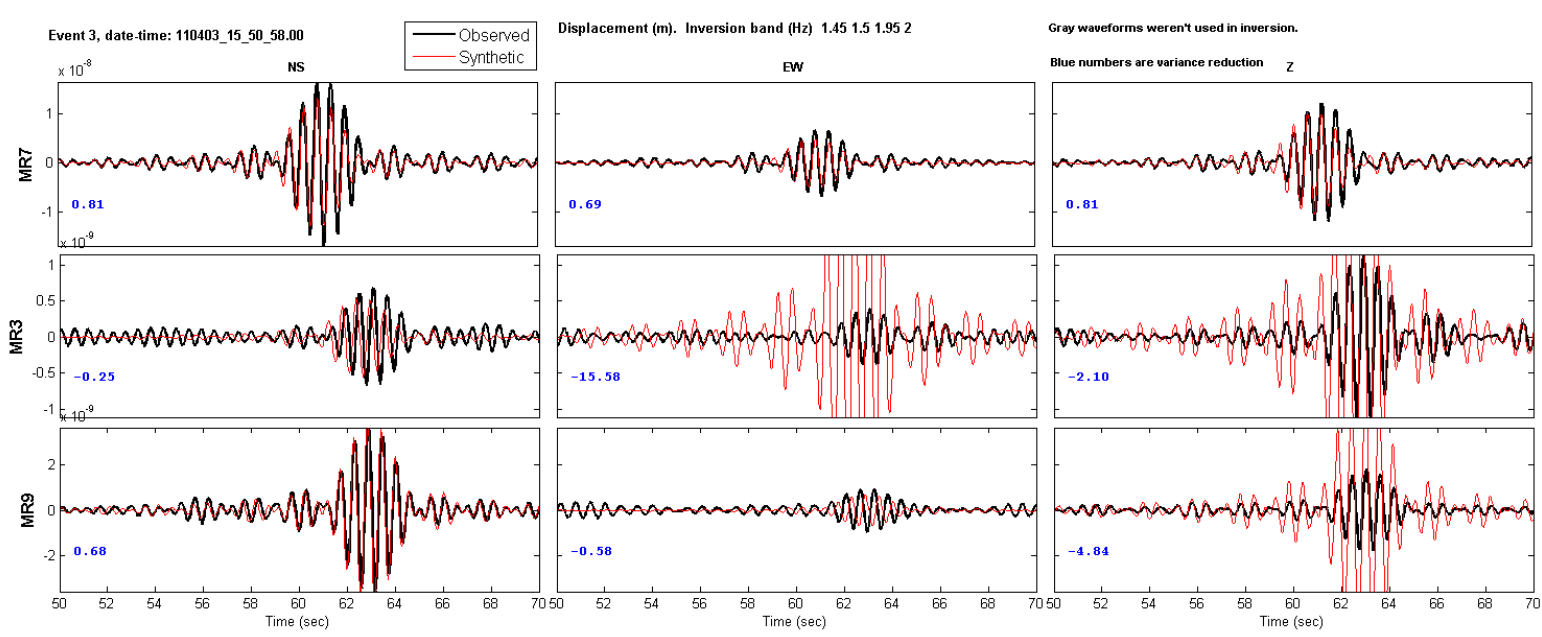

Figure II.3 - Comparison between the observed (black) and synthetic (red) waveforms. The plot refers to Event 3 of Tables 3.2 and 3.3. The waveform match is quantified by the variance reduction values per station components (blue numbers). The horizontal time axis is in seconds, $t=50 \mathrm{sec}$ corresponds to 10 seconds after the origin time. The vertical axis indicates the displacement amplitude in meters.
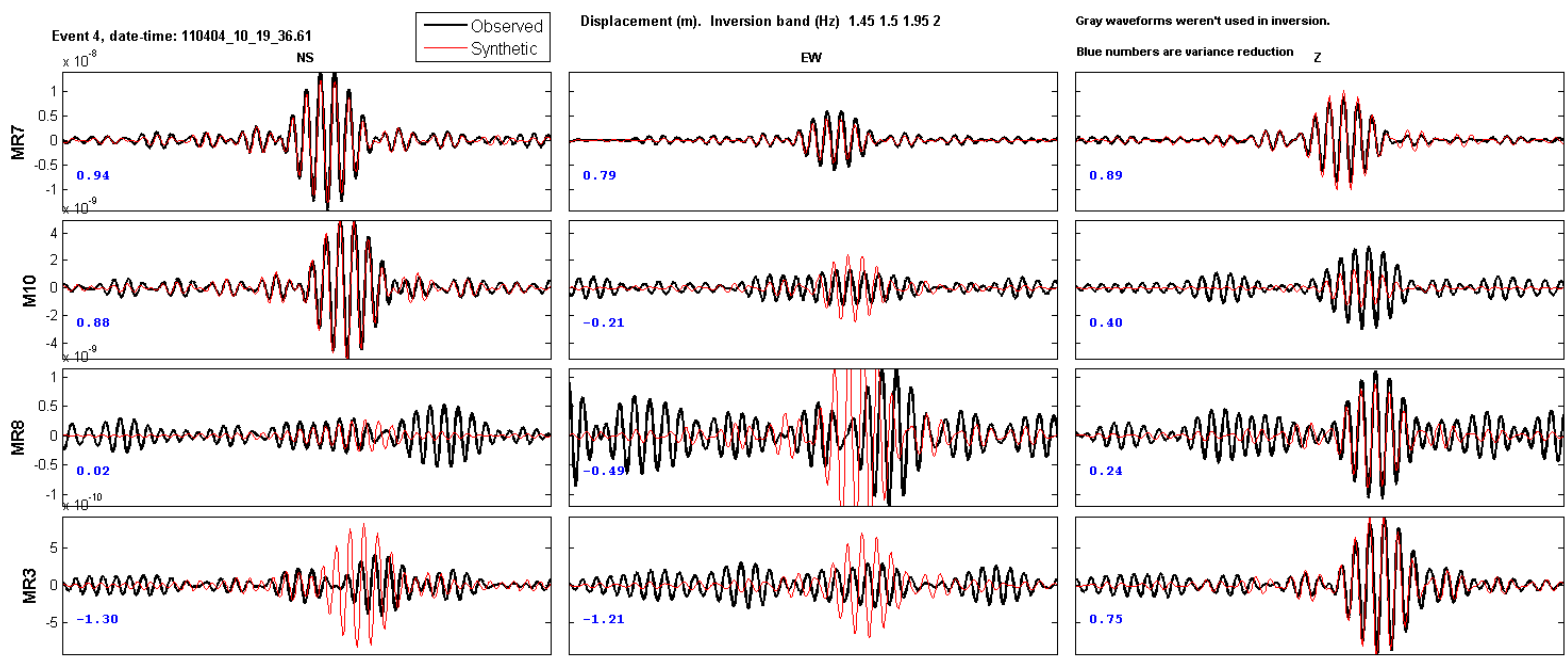

Figure II.4 - Comparison between the observed (black) and synthetic (red) waveforms. The plot refers to Event 4 of Tables 3.2 and 3.3. The waveform match is quantified by the variance reduction values per station components (blue numbers). The horizontal time axis is in seconds, $t=50 \mathrm{sec}$ corresponds to 10 seconds after the origin time. The vertical axis indicates the displacement amplitude in meters. 


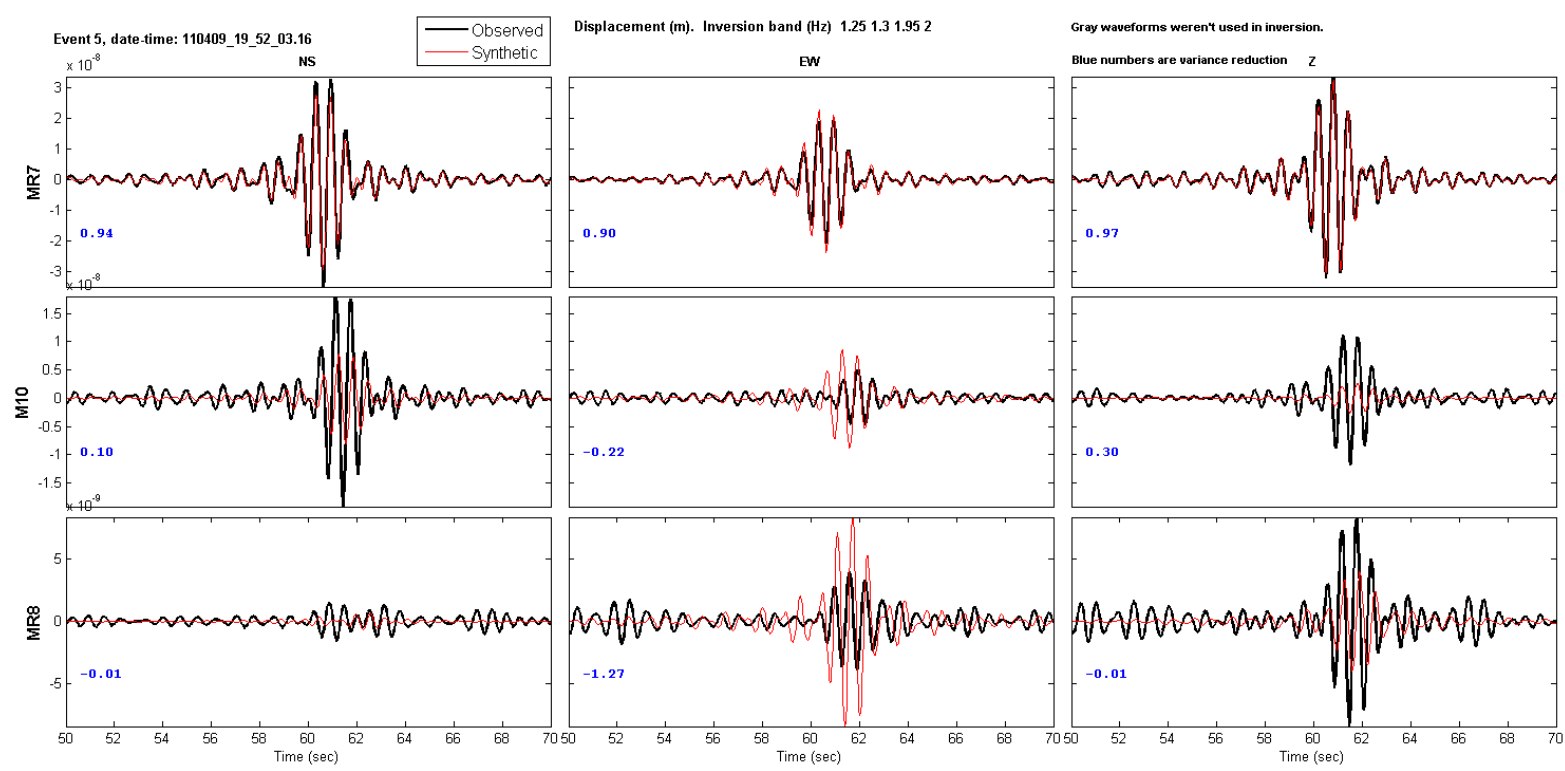

Figure II.5 - Comparison between the observed (black) and synthetic (red) waveforms. The plot refers to Event 5 of Tables 3.2 and 3.3. The waveform match is quantified by the variance reduction values per station components (blue numbers). The horizontal time axis is in seconds, $t=50 \mathrm{sec}$ corresponds to 10 seconds after the origin time. The vertical axis indicates the displacement amplitude in meters.
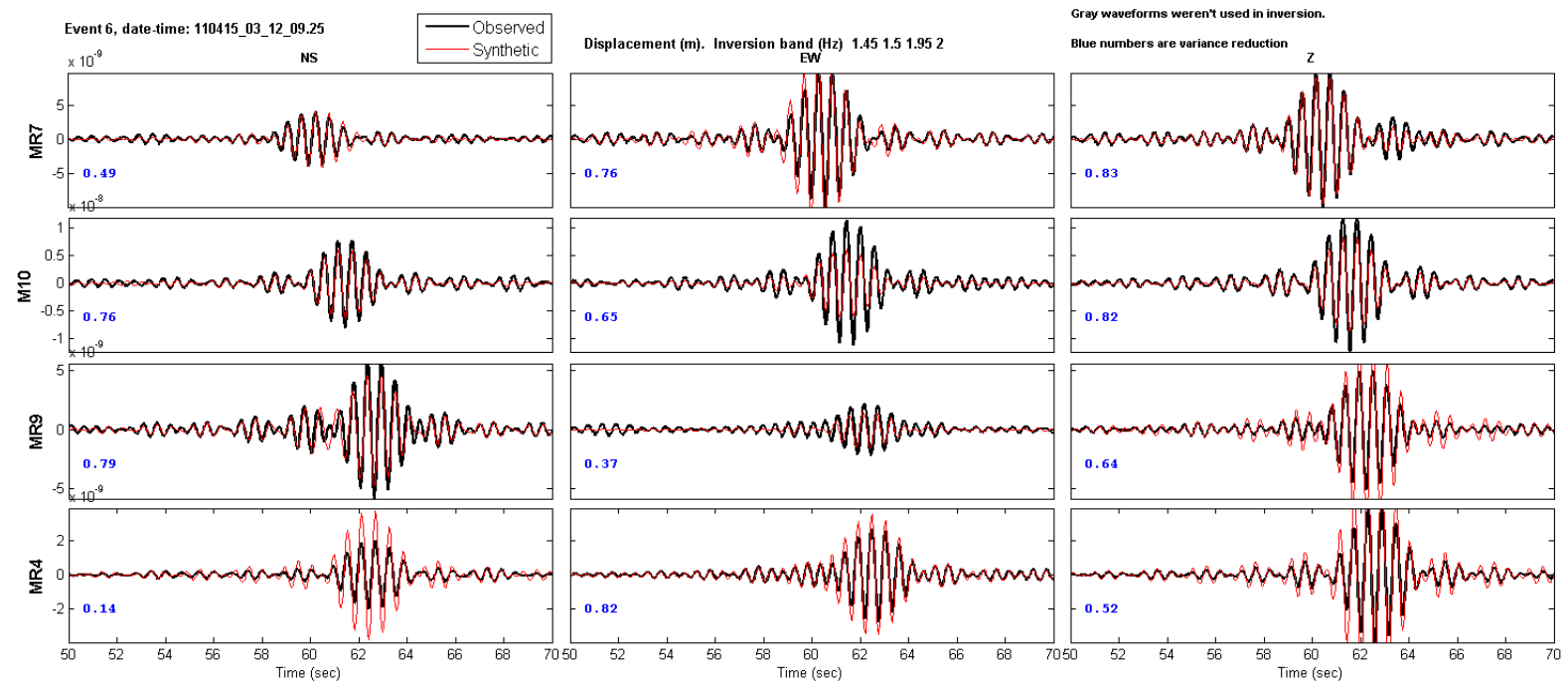

Figure II.6 - Comparison between the observed (black) and synthetic (red) waveforms. The plot refers to Event 6 of Tables 3.2 and 3.3. The waveform match is quantified by the variance reduction values per station components (blue numbers). The horizontal time axis is in seconds, $t=50 \mathrm{sec}$ corresponds to 10 seconds after the origin time. The vertical axis indicates the displacement amplitude in meters. 

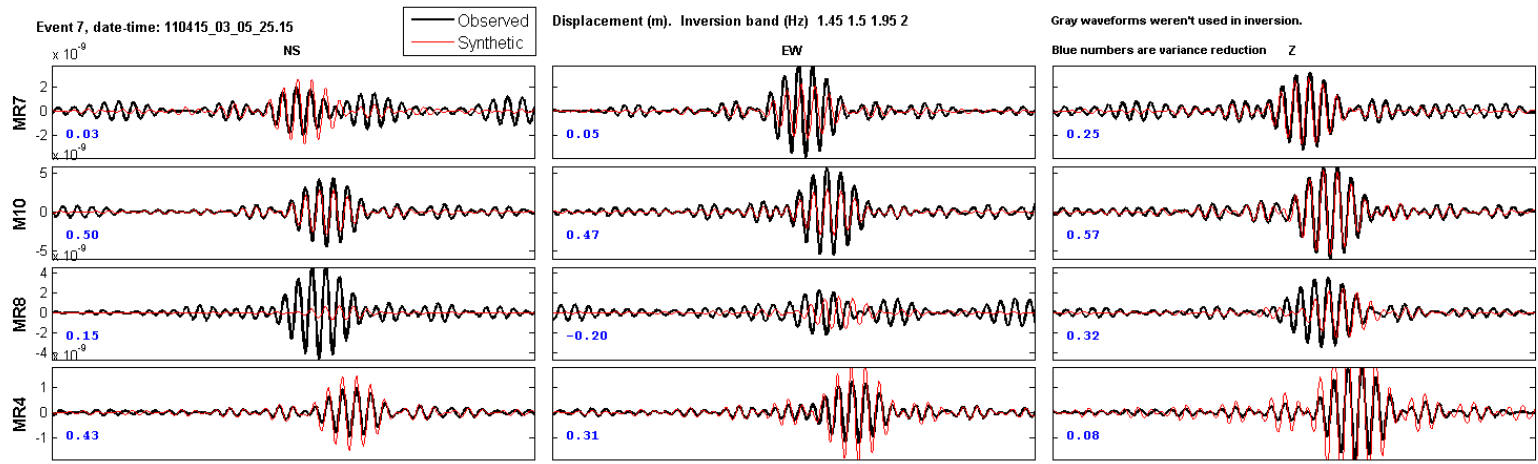

Figure II. 7 - Comparison between the observed (black) and synthetic (red) waveforms. The plot refers to Event 7 of Tables 3.2 and 3.3. The waveform match is quantified by the variance reduction values per station components (blue numbers). The horizontal time axis is in seconds, $t=50 \mathrm{sec}$ corresponds to 10 seconds after the origin time. The vertical axis indicates the displacement amplitude in meters.

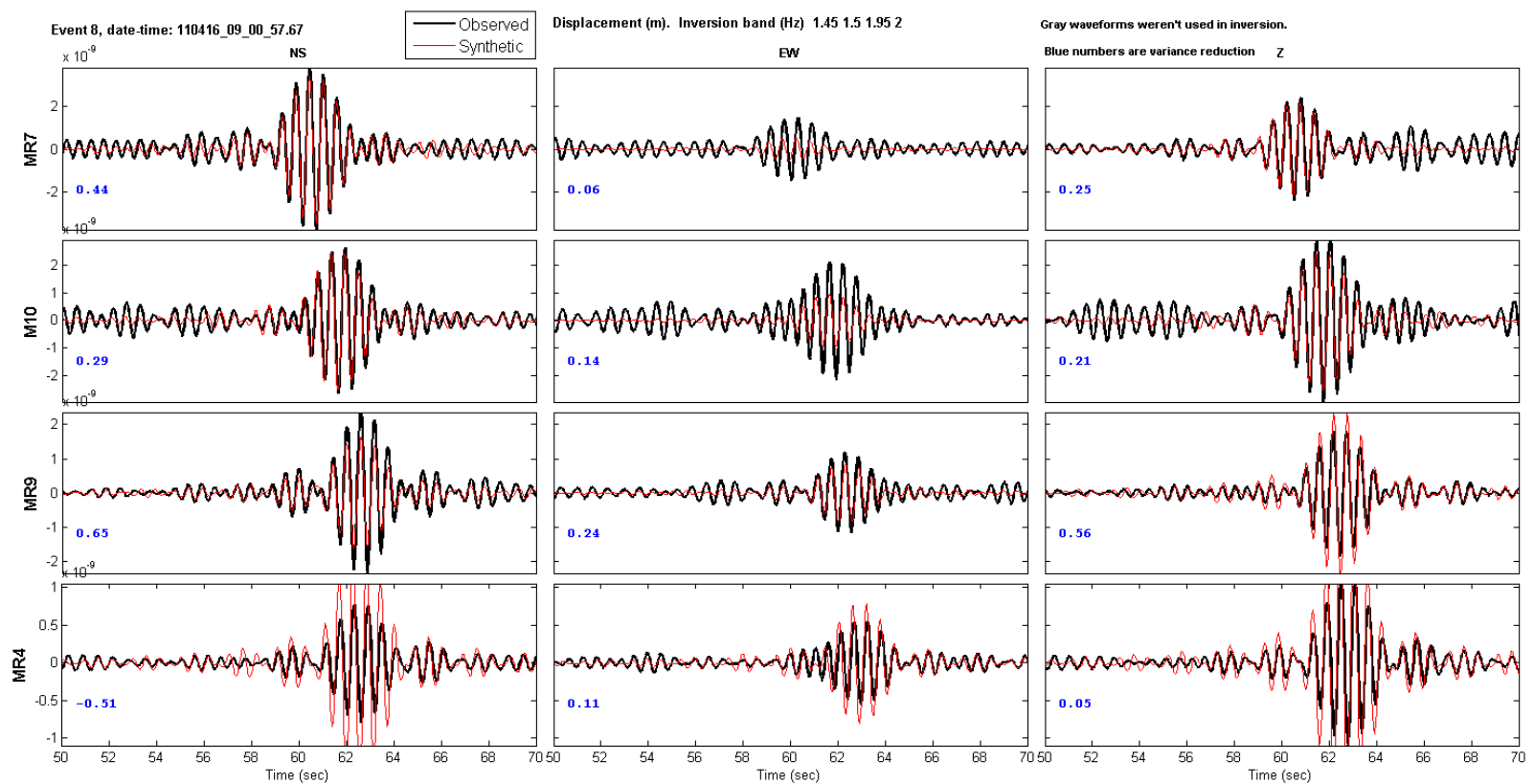

Figure II.8 - Comparison between the observed (black) and synthetic (red) waveforms. The plot refers to Event 8 of Tables 3.2 and 3.3. The waveform match is quantified by the variance reduction values per station components (blue numbers). The horizontal time axis is in seconds, $t=50 \mathrm{sec}$ corresponds to 10 seconds after the origin time. The vertical axis indicates the displacement amplitude in meters. 


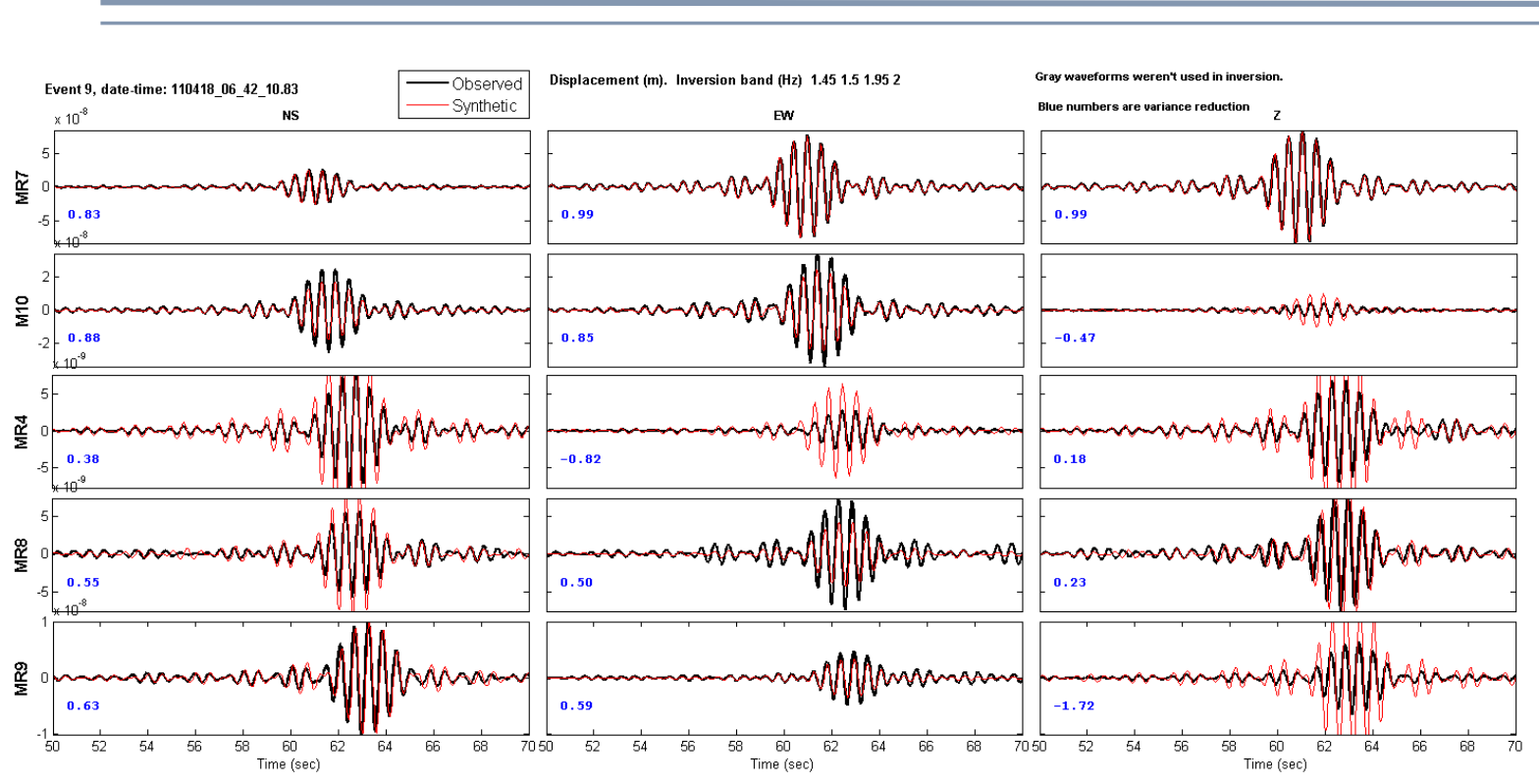

Figure II.9 - Comparison between the observed (black) and synthetic (red) waveforms. The plot refers to Event 9 of Tables 3.2 and 3.3. The waveform match is quantified by the variance reduction values per station components (blue numbers). The horizontal time axis is in seconds, $t=50 \mathrm{sec}$ corresponds to 10 seconds after the origin time. The vertical axis indicates the displacement amplitude in meters.
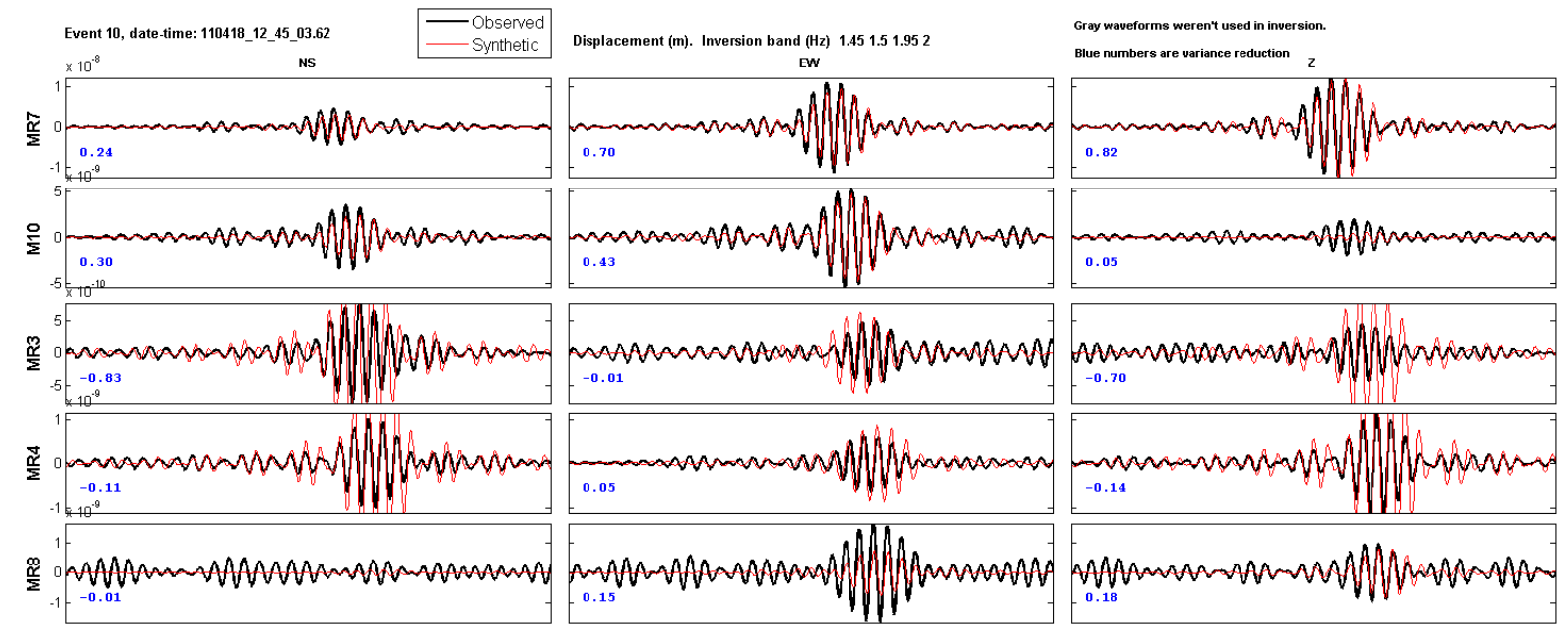

Figure II.10 - Comparison between the observed (black) and synthetic (red) waveforms. The plot refers to Event 10 of Tables 3.2 and 3.3. The waveform match is quantified by the variance reduction values per station components (blue numbers). The horizontal time axis is in seconds, $t=50 \mathrm{sec}$ corresponds to 10 seconds after the origin time. The vertical axis indicates the displacement amplitude in meters. 


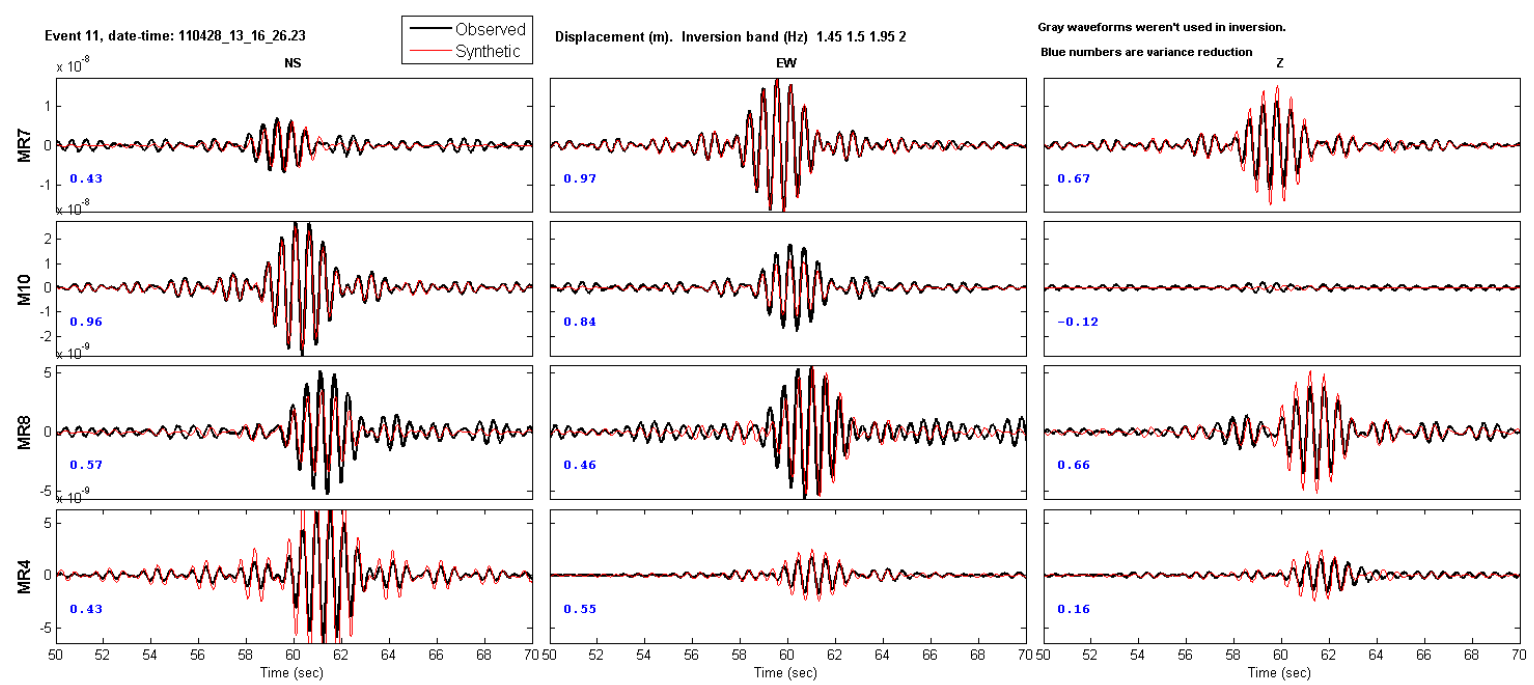

Figure II.11 - Comparison between the observed (black) and synthetic (red) waveforms. The plot refers to Event 11 of Tables 3.2 and 3.3. The waveform match is quantified by the variance reduction values per station components (blue numbers). The horizontal time axis is in seconds, $t=50 \mathrm{sec}$ corresponds to 10 seconds after the origin time. The vertical axis indicates the displacement amplitude in meters. 